\title{
SOFTWARE ENGINEERING TECHNIQUES
}

Report on a conference sponsored by the

\section{NATO SCIENCE COMMITTEE}

Rome, Italy, 27th to 31st October 1969

Chairman: Professor P. Ercoli

Co-chairman: Professor Dr. F. L. Bauer

Editors: J. N.Buxton and B. Randell

April 1970 
The present report is available from:

Scientific Affairs Division

NATO

Brussels 39

Belgium

Publishers:

NATO Science Committee

Printed in England at The Kynoch Press, Birmingham

Note for the current edition: The version of this report that you are reading was prepared by scanning the original edition, conversion to text through OCR, and then reformatting. Every effort has been made to do this as accurately as possible. However, it is almost certain that some errors have crept in despite best efforts. One of the problems was that the OCR software used kept trying to convert the original British spellings of words like 'realise' to the American spelling 'realize' and made other stupid mistakes. Whenever the OCR program was unsure of a reading, it called it to the attention of the operator, but there were a number of occasions in which it was sure, but wrong. Not all of these instances are guaranteed to have been caught.

Although the editor tried to conform to the original presentation, certain changes were necessary, such as pagination. In order that the original Table of Contents and Indices would not have to be recalculated, an artifice was used. In the PDF version, the original page breaks are indicated in the text thusly: 499 indicates that this is the point at which page 49 began in the original edition. If two such indicators appear together, this shows that there was a blank page in the original.

The figures have been redrawn to improve legibility. The original size and scale was not changed. In order to accommodate the new pagination, the figures may have been shifted slightly from their position in the original document.

Finally, it should be noted that the effort required to produce the current edition was a tiny fraction of the effort required for the original. The current editor therefore wants to express his appreciation to the original editors, John Buxton and Brian Randell, for producing this second volume of NATO Conference Reports.

Robert M. McClure

Arizona 2001 


\section{CONTENTS}

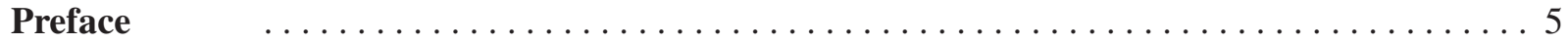

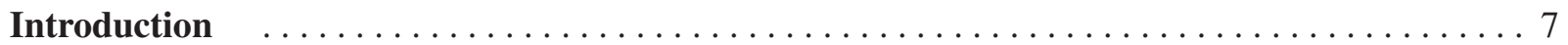

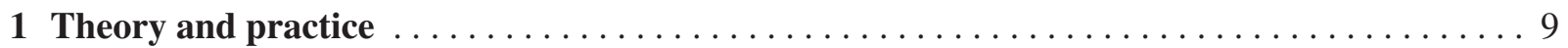

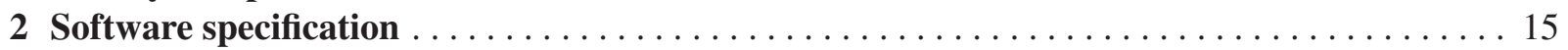

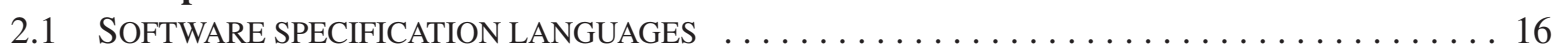

2.2 SOFTWARE IMPLEMENTATION LANGUAGES $\ldots \ldots \ldots \ldots \ldots \ldots \ldots \ldots \ldots \ldots \ldots \ldots \ldots \ldots$

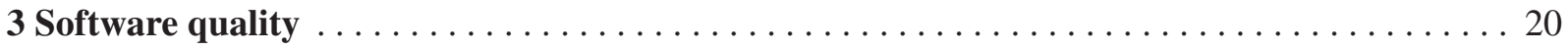

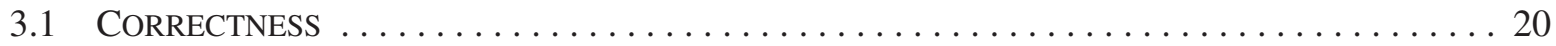

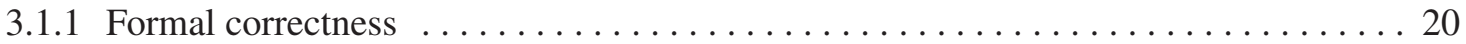

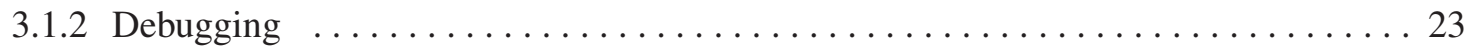

3.1.2.1 On classical techniques . . . . . . . . . . . . . . . . . . . . 23

3.1.2.2 On on-line and off-line techniques .................... 24

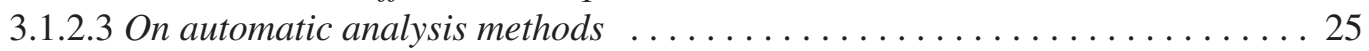

3.2 PERFORMANCE MEASUREMENT AND IMPROVEMENT $\ldots \ldots \ldots \ldots \ldots \ldots \ldots \ldots \ldots \ldots \ldots$

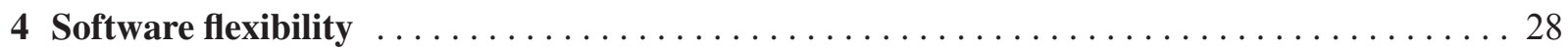

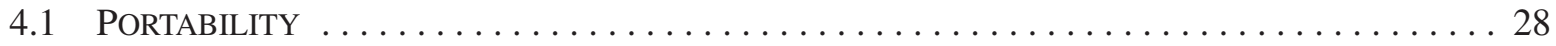

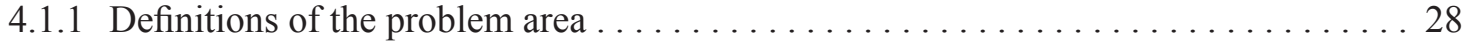

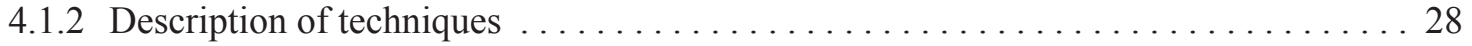

4.1.2.1 High level language support . . . . . . . . . . . . . . . . . . . 29

4.1.2.2 Bootstrap from the old system . . . . . . . . . . . . . . . . 29

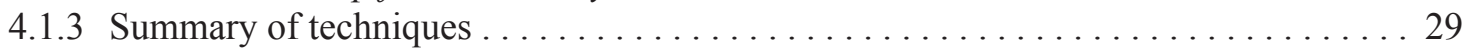

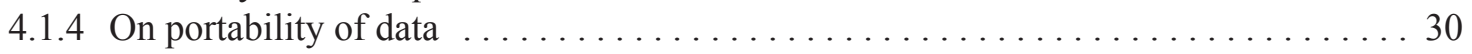

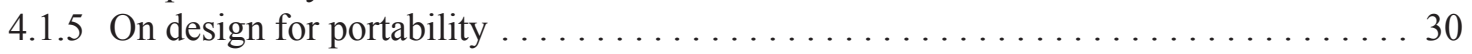

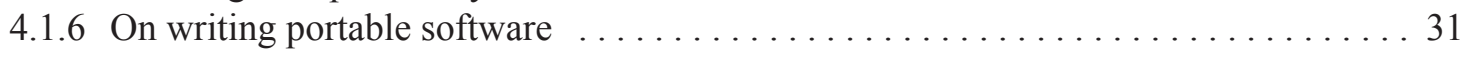

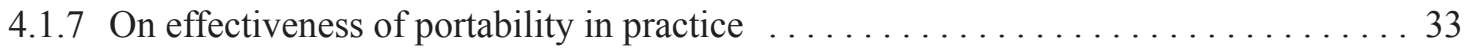

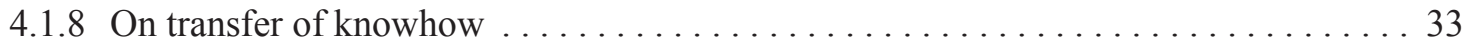

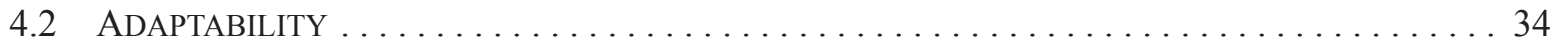

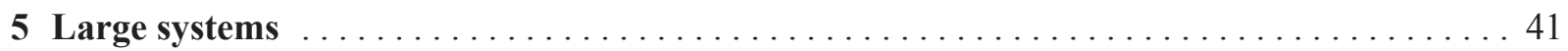

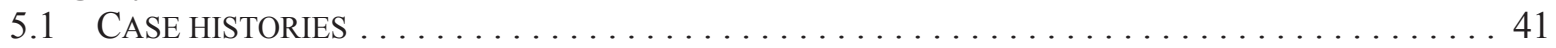

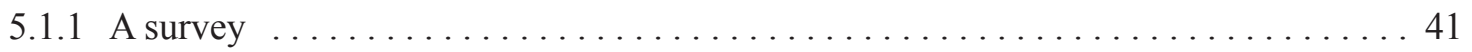

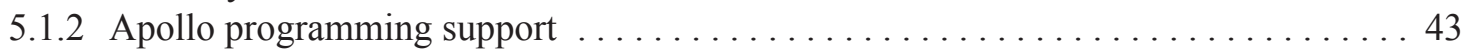

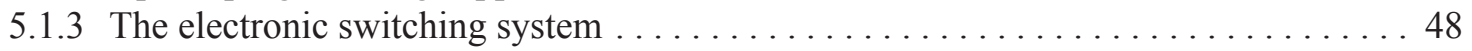

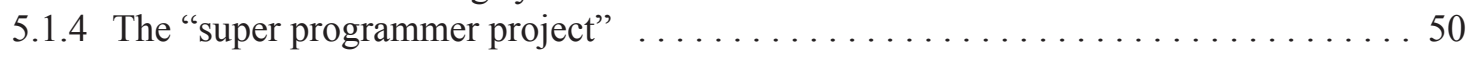

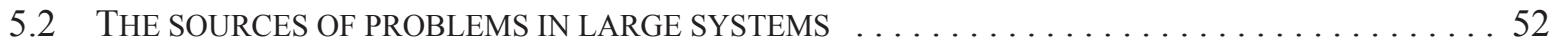

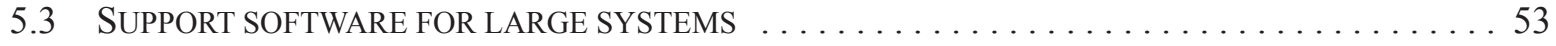

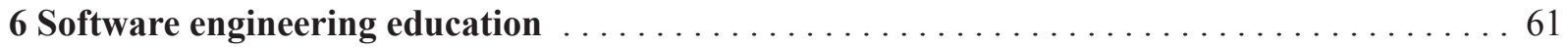

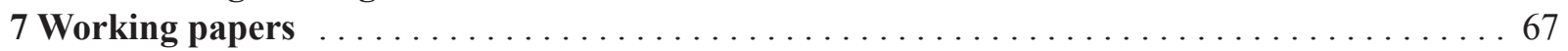

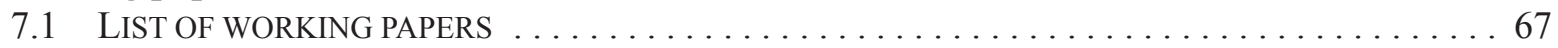

7.2 J. D. Aron: Estimating resources for large programming Systems $\ldots \ldots \ldots \ldots \ldots 6$

$7.3 \quad$ W. S. Brown: Software portability $\ldots \ldots \ldots \ldots \ldots \ldots \ldots \ldots \ldots \ldots \ldots \ldots \ldots \ldots$

7.4 E. W. Dijkstra: Structured programming $\ldots \ldots \ldots \ldots \ldots \ldots \ldots \ldots \ldots \ldots \ldots \ldots \ldots . \ldots 4$

7.5 A. D. Falkoff: Criteria for a system design language $\ldots \ldots \ldots \ldots \ldots \ldots \ldots \ldots \ldots \ldots 88$

7.6 C. C. Gotlieb and G. H. McEwen: System evaluation tools . . . . . . . . . . . . . . . 93

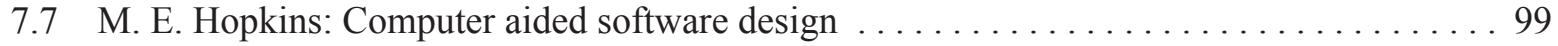

7.8 C. A. Lang: Languages for writing system programs $\ldots \ldots \ldots \ldots \ldots \ldots \ldots \ldots \ldots \ldots \ldots$

7.9 J. M. MacGowan, Jr..: UNIVAC 1108 instrumentation $\ldots \ldots \ldots \ldots \ldots \ldots$ 
7.10 R. M. Needham: Software engineering techniques and operating system

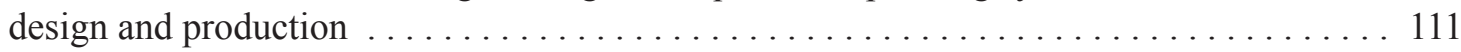

7.11 R. M. Needham and J. D. Aron: Software engineering and computer

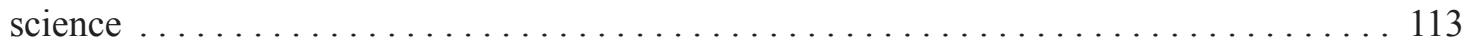

7.12 H. Schorr: Compiler writing techniques and problems $\ldots \ldots \ldots \ldots \ldots \ldots \ldots \ldots \ldots \ldots$

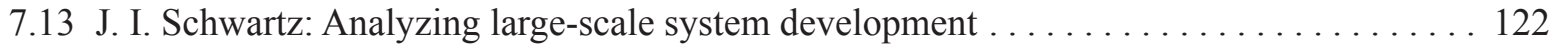

$7.14 \mathrm{~W}$. Teitelman: Toward a programming laboratory $\ldots \ldots \ldots \ldots \ldots \ldots \ldots \ldots \ldots \ldots \ldots$

7.15 W. Ulrich: Design of high reliability continuous operation systems . . . . . . . . . . . . 149

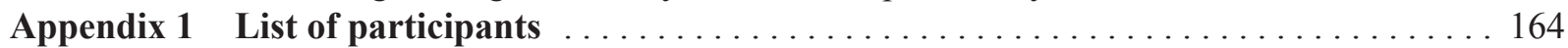

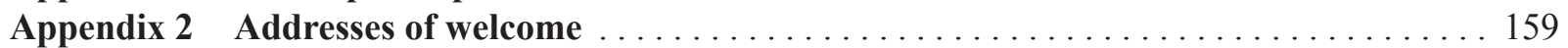

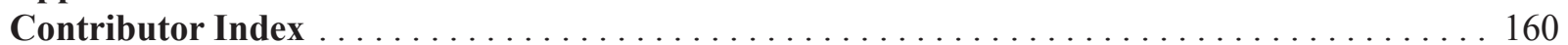

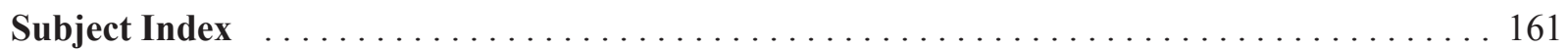




\section{PREFACE}

A working conference on software engineering techniques, sponsored by the NATO Science Committee, was held from the 27th to 31st October 1969, near Rome, Italy. The conference was intended as a direct sequel to the NATO conference on software engineering held at Garmisch, Germany, from 7th to 11th October 1968. About sixty people from eleven countries attended the meeting. A list of participants is provided in Appendix 1.

This report summarizes the discussions held at the conference and includes a selection from the more than 50 working papers prepared by the conference participants. The report has been prepared in much the same manner as was used for the report of the Garmisch conference, and which is described in the preface to that report.

Material from the working papers and from transcribed and edited discussions has been combined under specific headings into sections 1 to 6 of this report. Lengthy working papers that have been selected for the report are reproduced in section 7.

Two sessions at the conference, on the subject of the NATO Science Committee proposals for an International Institute for Software Engineering, which were in the main nontechnical, have not been covered in this report.

The similarities of the structure of this report to that of its predecessor are to a certain extent superficial, owing to the fact that the Rome conference turned out to be rather different in form from the Garmisch conference. The reasons for this, and the effect that they have had on the report are discussed in the introduction. The opinions expressed in the introduction are entirely the responsibility of the editors. In the rest of the report the editors have endeavoured to restrict their role to that of selection and classification of material.

As with the Garmisch report, readers should keep in mind the fact that all those present at the meeting expressed their views as individuals and in no sense as representatives of the organizations with which they are affiliated.

The actual work on the report was a joint undertaking by several people. The large amounts of typing and other office chores were done by Miss Margaret Chamberlin and Miss Ann Laybourn. During the conference notes were taken by Rod Ellis and Ian Hugo, who also operated the tape recorders, and by John Buxton and Brian Randell. The reviewing and sorting of material for the report, and the final write-up were done by John Buxton and Brian Randell assisted by Rod Ellis and Ian Hugo. The final version of the report was prepared by The Kynoch Press, using their computer typesetting system (see Cox, N. S. M. and Heath, W. A.: 'The integration of the publishing process with computer manipulated data'. Paper presented to the Seminar on Automated Publishing Systems, 7-13th September 1969, University of Newcastle upon Tyne, Computer Typesetting Research Project), the preliminary text processing being done using the Newcastle File Handling System (see the paper by Cox and Dews in: Cox, N. S. M. and Grose, M. W. (editors): 'Organization and handling of bibliographic records by computer', 1967, Newcastle upon Tyne, Oriel Press Ltd.). Keypunching of the text and the composition control codes was done by Ron White of The Kynoch Press as part of a study undertaken with the support of the National Graphical Association. The computer time was 6 generously provided by Professor E. S. Page, Director of the Computing Laboratory of the University of Newcastle upon Tyne. During the conference photocopying equipment was provided by the Dupleco Company.

One quotation from the discussion during the closing section of the conference seems appropriate to end this preface.

Randell: Writing this sort of report is like building a big software system. When you've done one you think you know all the answers and when you start another you realize you don't even know all the questions.

John Buxton

Brian Randell 


\section{INTRODUCTION}

The Rome conference on software engineering techniques was intended as a direct sequel to the conference on software engineering held in Garmisch, Germany, 7th to 11th October 1968. The Rome conference took on a form rather different from that of the conference in Garmisch and hence the resemblance between this report and its predecessor is somewhat superficial. The role played by the editors has changed and this change deserves explanation.

The Garmisch conference was notable for the range of interests and experience represented amongst its participants. In fact the complete spectrum, from the inhabitants of ivory-towered academe to people who were right on the firingline, being involved in the direction of really large scale software projects, was well covered. The vast majority of these participants found commonality in a widespread belief as to the extent and seriousness of the problems facing the area of human endeavour which has, perhaps somewhat prematurely, been called "software engineering". This enabled a very productive series of discussions, in both plenary and informal parallel sessions, to take place. During these the goal was to identify, classify, and discuss the problems, both technical and managerial, which face the various different classes of software projects, up to and including the type of projects which can only be measured in man-millenia. Also, the fact that the goal of the conference was to produce a report was always kept in mind. As a result, at the end of the conference, the editors (Peter Naur and Brian Randell), had a comparatively clear idea of what the conference participants hoped for from the report and a detailed initial draft of the structure that the report should have. The role of the editors could therefore be largely restricted to that of selecting and classifying statements made by participants in working papers or at the conference.

The intent of the organizers of the Rome conference was that it should be devoted to a more detailed study of technical problems, rather than including also the managerial problems which figured so largely at Garmisch. However, once again, a deliberate and successful attempt was made to attract an equally wide range of participants. The resulting conference bore little resemblance to its predecessor. The sense of urgency in the face of common problems was not so apparent as at Garmisch. Instead, a lack of communication between different sections of the participants became, in the editors' opinions at least, a dominant feature. Eventually the seriousness of this communication gap, and the realization that it was but a reflection of the situation in the real world, caused the gap itself to become a major topic of discussion. Just as the realization of the full magnitude of the software crisis was the main outcome of the meeting at Garmisch, it seems to the editors that the realization of the significance and extent of the communication gap is the most important outcome of the Rome conference.

In view of these happenings, it is hardly surprising that the editors received no clear brief from the conference as to the structure and content of the report. It seemed to us that the most useful discussions centered around specific problem areas in need of solution rather than specific techniques in search of problem areas. We have therefore structured the report accordingly.

8

The problem areas into which we have classified the material are clearly neither all of the same importance, nor at similar states of development, nor even completely independent of each other. Rather, they constitute, in our opinion, a convenient classification of the discussions and the working material submitted.

Some of the material presented either in working papers or in discussion related directly to areas of the subject that were extensively discussed at Garmisch, such as various managerial and organizational problems. In general this material has not been included unless, in our view, it has substantially extended or thrown new light upon these areas. Furthermore much working material concerned subjects which were not discussed at the conference. In the absence of the guidance which such discussions would have given us, we chose in general not to include such material in this report. The material submitted by the editors themselves was in fact rejected on these grounds. 


\section{THEORY AND PRACTICE}

Most of the material in this section is collected from a discussion which was held on the last day of the conference after many people had expressed the need for the conference to talk about, rather than just suffer from, the effects of the communication gap.

Strachey: I want to talk about the relationship between theory and practice. This has been, to my mind, one of the unspoken underlying themes of this meeting and has not been properly ventilated. I have heard with great interest the descriptions of the very large program management schemes, and the programs that have been written using these; and also I heard a view expressed last night that the people who were doing this felt that they were invited here like a lot of monkeys to be looked at by the theoreticians. I have also heard people from the more theoretical side who felt that they were equally isolated; they were here but not being allowed to say anything. We have seen a document by Aron and Needham essentially about "Can computing science be of any assistance to software engineering, or what can software engineering get out of computing science?" (see section 7.11-Eds.) One can also ask the question the other way round: "What can computing science get out of software engineering?"

This sort of debating point is not helpful. The truth of the matter is that we tend to look with doubt and suspicion at the other side; whichever side of that particular barrier we are. On one side we say "Well, there's nothing we can get out of computing science: look at all this rubbish they are talking". Or we stand on the other side and look at the very large programs and we say "Goodness me; what rotten techniques they use and look: they all fail!"

One of the most interesting things that has been shown at this conference is that these projects don't all fail. It has been shown that some of them have been quite astonishingly successful. I think we ought to remember somebody or other's law, which amounts to the fact that 95 per cent of everything is rubbish. You shouldn't judge the contributions of computing science to software engineering on the 95 per cent of computing science which is rubbish. You shouldn't judge software engineering, from the high altitude of pure theory, on the 95 per cent of software engineering which is also rubbish. Let's try and look at the good things from the other side and see if we can't in fact make a little bridge. Let's see what the real difficulties are and whether we can do something to assist.

It seems to me that one of the difficulties about computing science at the moment is that it can't demonstrate any of the things that it has in mind; it can't demonstrate to the software engineering people on a sufficiently large scale that what it is doing is of interest or importance to them. Take a very simple example with which I think almost everybody in the small scale and more abstract way of thinking about programming would agree; that to use recursive methods is undoubtedly an assistance. No self respecting programmer would dream of programming in a language without recursion. Every large-scale complicated problem (and by large-scale, in this case, I mean something that one person can do) is greatly aided by the use of recursive methods; I think there is no question whatsoever that this is true $\mathbf{1 0}$ and it has been amply demonstrated by all sorts of individual programming efforts. Now so far as I know recursive programming is not used in general in any large-scale software system, with a few exceptions such as the Burroughs people. In fact the statement seems to be completely true about all really large systems. They use methods which do not allow them to use recursion; I think the real reason for this is that they haven't thought seriously of doing it. They've been told to do it and they brush it away apparently because it hasn't got the right sort of software support or because their machine doesn't do it easily or because they don't know about it. How can we convince people who are dealing with hundreds of programmers and millions of instructions that something as radical as changing the basic core of the way in which they program is a good thing to do?

Clearly you can't expect anybody to change a very large project completely in a direction like that merely because you say it's a good idea. This is obviously nonsense. On the other hand it's equally impractical to ask a university department or even a technical institution to mount a demonstration project of 500 man years; they haven't got the money and they wouldn't know how to manage it anyhow. They have no managerial capabilities. 
Incidentally, we all know that many of the operating systems and specialist compilers and things that are written in universities appear to be written with very little effort and in very little time compared with some of the commercial ones. It is very important to realize that the result is still an amateur affair; it is not documented, it is not maintained, it is not maintainable and when the man who wrote it goes away, it collapses. There is a very great deal of difference between the amateur work which is done in the university and the professional work which is done by people who have very large-scale things to do and have commercial responsibilities.

We need something in the way of a proving ground, halfway between the very large projects which must rely on things that the managers already know how to use, and the more advanced techniques which we're quite sure are all right on a small scale but which we still have to develop on a somewhat larger scale. We need as it were a pilot plant and until we can do something like this we shall not be able to introduce into software engineering the speed of development change which is necessary for it to keep up with the extremely rapidly developing theoretical advances in the subject. I don't know how this ought to be done; a development pilot plant is an expensive operation and cannot be done by universities alone. It could be done, I think, by a co-operative effort between the manufacturers, who have the financial resources and the interest in very large systems, and the research institutions. It does require a pilot plant which has to construct a really fairly sizeable thing; a one man exercise redoing a job in a quarter of the time or tenth of the time or whatever is not good enough. You must build a reasonably sized system where management problems will arise and can be demonstrated to be soluble and where new techniques can be used by managers. You must have at least part of the real difficulty of the big problems.

The thing that saddens me about the present situation is that there's not much sign that the large engineering setups have yet been able to change their basic techniques. They can change the peripheral stuff, the editing and the documentation and things like this, but so far they haven't changed the central core of what they are doing: that is to say, the actual programming techniques. Right in the middle it is still, as somebody said the other day, classical programming and classical mistakes. FORTRAN was a very good language when it started but that was a long time ago; it's at least half the 11 lifetime of this subject ago, and that is a long time. If in any other kind of engineering you used techniques that were half the life time of the subject old, you would be very much out of date.

Now I want to preach to people on the other side. It seems to me that the academics have been disgracefully arrogant in neglecting and denying the existence of large problems. For years and years they refused to admit that commercial data processing was a subject which had any intellectual interest at all. It was expressed in horrible language: COBOL is, as most people would agree, a horrible language; it leads to dreadful things like saying DIVIDE CAKE INTO 3 which means you get "three over the cake" and not "the cake over three". In fact commercial data processing had and still has very interesting problems about data structuring, which it had to deal with and started dealing with long before the academic side of programming would have anything to do with it. None of the early academic programming languages have data structures. The reason is that the academics just could not be bothered to look at this large, important area.

I think the same thing is true about very large, complicated programs; it seems to be quite clear that the large software engineering exercises introduce new and important ideas which we must acknowledge. For instance, how are we going to organize programs intellectually, which are so large that they can't be comprehended by one man? This is a very important problem. It's clear to me, or at least I think it's clear to me, that the only hope of doing this is by arranging these very large programs in hierarchical ways. It's extremely important that we should begin on the theoretical task of trying to understand how to mould messy, difficult and inconvenient objects into hierarchical structures. Another principle that comes from study of large engineering projects is that a great deal of the effort must be spent in dealing with malfunctions of some sort: program malfunctions, machine malfunctions or operator malfunctions.

These are things that are awkward to get into an elegant mathematical framework. Nevertheless we must somehow or other form a conceptual framework in which we can talk about these things in a clean and comprehensible way. I think we've got a lot to learn here; I don't know how to do it, I'm just beginning to think about it and I think it's very exciting.

Aron: Part of the gap that has been mentioned here between computer science and software engineering is the recognition that many software engineering projects start and proceed without the planned utilization of scientific

\section{NATO SOFTWARE ENGINEERING CONFERENCE 1969}


methods. The fact is that if we look back we can see that many things were done wrong, but they were done wrong by people who thought they were doing the right thing.

Ross: What does a manager look for in a technique before he adopts it?

Aron: If he is engaged in an operational rather than an experimental project, he will insist that the technique is thoroughly tested; it must be shown to be reliable and shown to have performance parameters that are suitable. He acts on his own experience. He will adopt what you tell him only to the extent that you can demonstrate it to him.

Needham: How big does a project have to be to be a useful pilot project? It clearly can't be 500 men for five years; that's not a pilot project, it's a project. It clearly isn't a matter of one man for one year, because this is precisely what we say isn't good enough. Can we say what kind or size of pilot project, which would be done according to the best principles that 12 computer scientists could devise, would look in some way acceptable to people who are in the really large-scale business, and would stand a chance of being imitated or developed? If we had some answer we might be able to go away and do such a pilot project.

\section{Feldman: MULTICS!}

Ulrich: What is the difference between a pilot project, and an actual project?

Galler: One important difference is that there be no predetermined time limit specified by marketing people.

During the discussion a rather different analysis of the situation was proposed by Sharp.

Sharp: I think that we have something in addition to software engineering: something that we have talked about in small ways but which should be brought out into the open and have attention focused on it. This is the subject of software architecture. Architecture is different from engineering.

As an example of what I mean, take a look at OS/360. Parts of OS/360 are extremely well coded. Parts of OS, if you go into it in detail, have used all the techniques and all the ideas which we have agreed are good programming practice. The reason that OS is an amorphous lump of program is that it had no architect. Its design was delegated to a series of groups of engineers, each of whom had to invent their own architecture. And when these lumps were nailed together they did not produce a smooth and beautiful piece of software.

I believe that a lot of what we construe as being theory and practice is in fact architecture and engineering; you can have theoretical or practical architects: you can have theoretical or practical engineers. I don't believe for instance that the majority of what Dijkstra does is theory-I believe that in time we will probably refer to the "Dijkstra School of Architecture".

What happens is that specifications of software are regarded as functional specifications. We only talk about what it is we want the program to do. It is my belief that anybody who is responsible for the implementation of a piece of software must specify more than this. He must specify the design, the form; and within that framework programmers or engineers must create something. No engineer or programmer, no programming tools, are going to help us, or help the software business, to make up for a lousy design. Control, management, education and all the other goodies that we have talked about are important; but the implementation people must understand what the architect had in mind.

Probably a lot of people have experience of seeing good software, an individual piece of software which is good. And if you examine why it is good, you will probably find that the designer, who may or may not have been the implementer as well, fully understood what he wanted to do and he created the shape. Some of the people who can create shape can't implement and the reverse is equally true. The trouble is that in industry, particularly in the large manufacturing empires, little or no regard is being paid to architecture. 
An alternative to the "pilot project" proposal was put forward d by David.

David: It has been suggested that demonstration systems or systems $\mathbf{1 3}$ experiments are a good approach to solving the problems of large systems. But in many instances, I think, just the sheer size and cost of a demonstration and the difficulty of measurement and of generalization of the results prevents a convincing demonstration; it certainly prevents the conduct of a properly controlled experiment in many cases.

Another possibility for aiding technology transfer lies in studying in detail actual ongoing production efforts. I think a great deal of information on the influence of new methods on production can be gained by a bona fide analysis of project performance. A combination of that kind of hard information with selected experiments and demonstrations might be much more effective than either alone.

There is a great deal of uncertainty about the influence of new production techniques on cost, manpower and time, etc. This uncertainty, I think, rests on a profound lack of evidence and hard data about real cases. Techniques which are said to be the same succeed in some cases and they fail in others. That's no surprise to anybody but the crucial factors which actually contribute to the success or failure have not been successfully identified. They certainly have not been established quantitatively, even approximately. It is clear too, that many factors are involved in success and failure: many more than the discussions at various meetings or post-mortem papers after the end of a project would seem to imply.

Dijkstra I would like to comment on the distinction that has been made between practical and theoretical people. I must stress that I feel this distinction to be obsolete, worn out, inadequate and fruitless. It is just no good, if you want to do anything reasonable, to think that you can work with such simple notions. Its inadequacy, amongst other things, is shown by the fact that I absolutely refuse to regard myself as either impractical or not theoretical.

David expressed the idea that "We can make case studies in industry, and then you can study the results. You can do these analyses." A probable effect of the distinction is that if such a study is made, the output of it can be regarded as theory and therefore ignored. What is actually happening, I am afraid, is that we all tell each other and ourselves that software engineering techniques should be improved considerably, because there is a crisis. But there are a few boundary conditions which apparently have to be satisfied. I will list them for you:

1 We may not change our thinking habits.

2 We may not change our programming tools.

3 We may not change our hardware.

4 We may not change our tasks.

5 We may not change the organizational set-up in which the work has to be done.

Now under these five immutable boundary conditions, we have to try to improve matters. This is utterly ridiculous. Thank you. (Applause)

Randell: There is a well known English saying, which is relevant to this discussion about pilot projects. The saying is as follows: "There's none so blind as them that won't see". A pilot project will never be convincing to somebody who doesn't want to be convinced. A problem out in the real world will never be a problem to a theoretician who doesn't want to look at it. Blindness goes both ways. If you have people who are completely stuck in their own ways, whether these are ways of running large projects without regard for possible new techniques, or whether these are ways of concentrating all research into areas of ever smaller relevance or $\mathbf{1 4}$ importance, almost no technique that I know of is going to get these two types of people to communicate. A pilot project will just be something stuck in between them. It will be a Panmunjon with no way to it, from either side. You have to have good will. You have to have means for people to find out that what the others talk is occasionally sense. This conference may occasionally have done a little bit of that. I wish it had done a lot more. It has indicated what a terrible gulf we so stupidly have made for ourselves. 


\section{SOFTWARE SPECIFICATION}

The various problems concerned with obtaining a specification of a system, which would serve as the foundation of the subsequent system implementation, were debated at length. Much of the discussion arose in response to the following short working paper:

Seegmüller: (from "Definition of systems") "The following is a brief description of possible steps in which the definition of a system and the specification of an implementation of it might be achieved.

By a 'system' we understand a mechanism that is able to execute all of the functions given by its external interface.

1 Irrespective of hardware considerations, write in an informal way a list of all of the desired external functions and objects.

2 Put down performance limits for these functions.

3 Make a first guess whether there is a 'support system' (hardware or software system) which is likely to be capable of meeting the requirements listed under 1 and 2 . If the answer is no, then redesign under 1 and/or 2 or build a new support system.

4 Describe in an informal way an abstract machine (representing the system) which accepts invocations of all functions listed under 1 and can handle representations of the objects listed under 1 . If the result is unsatisfactory, then go to 1 .

5 Formalize the description of the abstract machine (e.g. by means of the Vienna definition method), remove possible contradictions and minimize the complexity of the abstract machine during this step. If the result is unsatisfactory, then go to 4 .

6 Use a software specification language (SSL) in order to define a first breakdown of the implementation of the abstract machine into program modules. Put down performance and size limits on these modules.

7 Use the resulting SSL statements in order to simulate the behaviour of these modules under the assumption of certain workload and support system characteristics. If the result is unsatisfactory, then redesign under 6 and repeat 7.

8 Refine the breakdown into modules.

9 Simulate the behaviour. If satisfactory, then go to 11.

10 Change the refinement. Go to 9.

11 If the level of detail is unsatisfactory, then go to 8 .

12 Take the last refinement as implementation specification."

Engeli: I think that this outline works well for small systems, where it is essentially unnecessary, but does not work, other than in exceptional cases, for large systems.

Oestreicher: No matter how precisely you try to specify a system, once you have built it you find it isn't exactly what is wanted. In real life, an outline like Seegmüller's must contain at all stages the possibility of returning to step 1.

Scalzi: My experience is with OS/360, and I agree with the criticisms of Seegmüller's implication that one must obtain a fully defined specification $\mathbf{1 6}$ before starting implementation. We have been building a growing system since 1966. By definition our design requirements are open-ended and our goal is to maintain a compatible interface to the user over a period of years. 
The discussion was in fact quite lengthy and tended in part to duplicate what had been said at Garmisch. However, several extended comments, describing experience of large system projects were made and are reported in section 5. There was much interesting discussion on the requirements of languages that could be used for specifying and/or implementing systems; this is reported below.

\subsection{Software specification languages}

The first discussion concerned the question of whether there need be a difference between languages used for specifying and for implementing software.

Seegmüller: I assume that a software specification language must be different from one that is suitable for implementation. I think that the Vienna Method is a most promising technique for formally specifying what the external behaviour is to be. It has been used so far for giving a formal definition of several programming languages, but there are now some attempts under way to apply it to operating systems.

Lowry: I think a major reason for a divergence between languages for these two purposes is the lack of sufficiently powerful optimization techniques.

Lucas: There is an important difference between a fully executable specification of a system and what we have done at Vienna. For example, the state of our abstract PL/1 machine has to be indeterminate where PL/1 does not specify a meaning to an action, or specifies only constraints. The axiomatic part of our scheme is an important part, and it is just not executable in any traditional sense.

Feldman: (from "Towards automatic programming") "The first problem one faces when trying to automate the writing of programs is this: how is one to say what is required without writing some kind of program? Workers in Artificial Intelligence have long faced this problem of process description and state descriptions. A state description for the function square root $(Z)$ might be:

The $\mathrm{X}$ such that $\mathrm{X} \times \mathrm{X}=\mathrm{Z}$

A process description for the same function might be an ALGOL program to carry out Newton's method for the solution. The state description above is much simpler than any process description, though this is not always the case. It is easier to describe how to take the derivative of a polynomial than to specify a set of properties that a derivative must have. Similarly, the syntax of a programming language is given more clearly by a grammar than by a set of conditions for wellformedness."

A different viewpoint was expressed by Falkoff, who thought that there was basically room for two languages: one being English or some other natural language and the other being a formal programming language. A paper by Falkoff on this subject, using examples from APL, is reproduced in section 7.5.

\section{7}

Perlis: In the past several years I have had periodic contacts lain with the APL people and I find that they are very much like both ardent Catholics and communists in that, when they get to certain topics, all discussions must be in their framework, otherwise no contact can be made. I wish to expose this as a virtue and not as a vice. There are several reasons why a COBOL compiler can be written in six months using APL as a specification language, as described by Ian Sharp. There is of course the fact that the APL primitives are very rich. However, there is something deeper than that, which is very important for software engineering. The APL people see all programming activities in terms of APL. When you talk about a table, they think in terms of APL algorithms for accessing and maintaining a table. Life is greatly simplified for them by virtue of the fact that all programming questions are recast into the framework of a language that they can carry around in their skulls. I have also observed this in the past with LISP programmers and it has been very important for them. 
Falkoff: What Perlis was saying is in part that, since we have finite skulls, we have to have reasonably concise representations of processes.

A related subject, which was never discussed at length but which was briefly referred to many times during the conference, concerned the interrelationships between program and data.

Schwartz: In my experience on large systems, we have pictured the systems as a flow of data. On these projects we have people whose sole job is to develop and to change and re-do table specifications. Wherever possible we try to have programming languages which divorce the data definition from the actual procedures. That happened to be the philosophy behind JOVIAL, which has been used for some number of these systems. Experimenting with the data flow specification forces an understanding as to what is going on in a system.

Randell: I am reluctant to draw a definite line between the concepts of program and data. One of the nice things about SIMULA is that the concept of process definition in some sense includes, as special cases, procedure and data structure definitions.

Further discussion related to this topic, but primarily concerning questions of program portability, is given in section 4.1.4. A working paper by Hopkins which is concerned with the central part played by data flow and table structures in determining system performance is given in full in section 7. 7.

\subsection{Software implementation languages}

Not surprisingly, there was a certain amount of discussion on programming languages at the conference.

Wirth: It might be thought quite remarkable what proportion of the submitted working material was on the subject of programming language development, although the conference is on software engineering. But in fact languages form our thinking habits and, are our daily tools, and I think it is fair to say that many of the problems plaguing the world of software are more or less directly attributable to the rather poor present state of language development.

18

One of the major points of discussion was the relationship between efficiency and naturalness of expression permitted by a programming language.

Wirth: The main objection to languages which are as distant from the actual computer hardware as the Galler and Perlis proposals, or APL, is that they are usually not very efficient. At the other end of the spectrum there are languages which reflect properties of the machine, so that you can get very efficient programs. I have produced languages belonging to each end of the spectrum. In the distant past I produced EULER, which proved to be an interesting academic exercise, and more recently, PL/360, which is nowadays quite widely used. Now if we are to talk about software engineering, we should talk about languages which have relevance to the reality of practical system development. A good engineering approach is to find a compromise which is as close to the machine as possible, while remaining machine independent.

Dijkstra: I would like to make a comment on Niklaus Wirth's story. I would like to point out that he starts from a tacit assumption which I wouldn't like to let pass unchallenged. His tacit assumption is that elegance or convenience on the one hand, and efficiency on the other hand, cannot go hand in hand ... I just don't believe it, and I would like to point out that from the point of view of Niklaus Wirth, his assumption is just a belief. If I have any interest in computing science at all it is based on my firm belief that very often the most attractive solution is the most efficient one. 
Feldman: (from "Towards automatic programming") "The search for more natural programming languages need not necessarily lead to less machine efficiency. The level of description found natural by people is often a natural one for code economization; the inclusion of extraneous detail proves a hardship to both man and machine."

Lampson: It seems to me that it is somewhat confusing to talk about PL/360 as a high level language. PL/360 is an assembler and that's worth bearing in mind. Many of the issues which arise in trying to decide whether some language which is not an assembler, such as MOL/940, is desirable or not, simply don't arise in connection with PL/360.

Falkoff: I would like to suggest a slight change in point of view, namely: that efficiency goes down as machines become more and more user independent. There is apparently a gap between the way people would like to think, and the way machines do things. To call this gap machine independence and put the onus on the user strikes me as looking at it backwards.

A fairly extensive discussion of the pros and cons of various languages which were designed for writing systems is given by Lang, in a paper which is given in full in section 7.8. At the conference he made the following plea.

Lang: It is an unfortunate fact that so many many systems are still written in assembly language. The perfect machine independent system writing language that we would like to have does not exist yet. On the other hand it is well within our present capability to design a simple machine dependent system writing language for any machine, and to write an efficient compiler 19 for it. We should make use of this capability. Further, a few machine independent system writing languages do exist, which deserve more use.

Buxton: I wish to respond to Charles Lang's plea for people to use simple programming languages more widely. This is a field which interests me because I have been gently growing one of these languages in my own backyard for the last four or five years, and by and large it's no better and no worse than any other such language, but it doesn't come into wide use other than with programmers in my own department. I think there is a quite subtle and rather fundamental reason for this. If you publish this sort of simple systems programming language, a system programmer will not use it because it is simple. He looks at it and says "This is so simple that I can design one of these myself this afternoon, so I'll dash one off and write a compiler in a week or two, rather than bother with somebody else's". It turns out that language design even of apparently trivial looking languages is a formidably difficult intellectual task - to do it right is very hard indeed. And unless he happens to be genuinely expert in language design he will end up with having produced a mess; a tiny mess, but nevertheless a mess. This psychological phenomenon prevents the use of other people's, sanitarily designed, systems programming languages. 


\section{SOFTWARE QUALITY}

The subject of software production and quality was covered in a broad sense at Garmisch. The purpose of this meeting was to concentrate in particular on the technical problems of software; having got a specification, the next problem is to produce a product which is in some sense of high quality. We have separated this section into two main areas; correctness of the product in performing the specified task (3.1) and its performance efficiency (3.2). The magnitude of the problem is indicated by the following quotations.

Hopkins: We face a fantastic problem in big systems. For instance, in OS/360 we have about 1000 errors each release and this number seems reasonably constant. Now why do we have these errors? Does this number relate in some way with the real number of errors in the system? It has been suggested that error reports come in at a rate connected with the system update release cycle; initially people don't report errors because of too many problems and towards the end of a release nobody reports errors as the new release will present a whole new problem. It is also suggested that the number of errors is directly linked to the number of field systems engineers. What sort of errors are made on large systems? What is the error rate in various modules? For instance, is it much higher in supervisor modules? Who makes the errors, new programmers or experienced ones? How long do errors stay in the system? These are very difficult, unanswered problems. There is also another theory: IBM have a large stock of new facilities back in Poughkeepsie waiting to be added to OS. Whenever the bug rate falls below 1000 per release, which seems to be about the user toleration level, they put in a few more facilities which has the effect of keeping the bug rate constant.

Schorr: It is not quite right that the rate is constant; it seems in fact to be slowly increasing!

\subsection{Correctness}

Section 3.1 is further subdivided into 3.1.1 on Formal Correctness (or, how to prevent bugs from ever arising) and 3.1.2 on Debugging Techniques (or, how to catch them when you have got them.)

\subsubsection{Formal Correctness}

Wirth: (from "The programming language PASCAL and its design criteria") "I believe that the art of computer programming is based on a relatively small number of fundamental concepts. It became clear during the development of PASCAL that most of these concepts are already present in mathematics in some form, usually surrounded by a terminology different from the one used by programmers. It is necessary to demonstrate this duality in the expression of concepts and to make the mathematician's knowledge of handling structures available to programmers. This knowledge 21 will be particularly useful for the development of techniques for program verification, i.e. for proving the correctness of programs. In fact, the idea of program verification has influenced the design of PASCAL considerably. Several commonly used features of other languages have been deliberately omitted from PASCAL, because they appear to be too sophisticated for presently known methods of proof. It became clear that those features which are least tractable to proof techniques are exactly the ones which are most difficult to define rigorously and which in practice present the most dangerous sources of programming error."

Feldman: (from "Towards automatic programming") "This question, correctness of programs, has been studied for some time and a number of significant results are being produced. One technique is to attach predicates to various parts of the program, the last one of which describes the desired result. Although there is no adequate survey of this material as yet, London has produced a comprehensive bibliography." (London R. L. 'Bibliography on proving the correctness of computer programs', Tech. Rep. No. 64, Computer Science Dept., University of Wisconsin, June 1969.) 
Hoare: One can construct convincing proofs quite readily of the ultimate futility of exhaustive testing of a program and even of testing by sampling. So how can one proceed? The role of testing, in theory, is to establish the base propositions of an inductive proof. You should convince yourself, or other people, as firmly as possible that if the program works a certain number of times on specified data, then it will always work on any data. This can be done by an inductive approach to the proof. Testing of the base cases could sometimes be automated. At present, this is mainly theory; note that the tests have to be designed at the same time as the program and the associated proof is a vital part of the documentation. This area of theoretical work seems to show a possibility of practical results, though proving correctness is a laborious and expensive process. Perhaps it is not a luxury for certain crucial areas of a program.

Perlis: Much of program complexity is spurious and a number of test cases properly studied will exhaust the testing problem. The problem is to isolate the right test cases, not to prove the algorithm, for that follows after the choice of the proper test cases.

Dijkstra: Testing shows the presence, not the absence of bugs.

Lucas gave a brief summary of the Vienna method (see P. Lucas and K Walk: "On the formal description of PL/1" Annual Review in Automatic Programming, Vol. 6). He gave an example of an application of the Vienna method, which revealed an error in the mechanism for dynamic storage allocation of AUTOMATIC variables in the F-level PL/I compiler (see P. Lucas: "Two constructive realizations of the block concept and their equivalence" TR 25.085; also W. Hanahpl: "A proof of correctness of the reference mechanism to automatic variables in the F-Compiler" LN 25.3.048; both of which are available from the IBM Vienna Laboratory).

Lucas: The error was not found by the compiler writers; it was not found by product test and it would not easily have been found by any random tests. I am quite convinced that making this proof was cheaper than the discussions I have heard among highly-paid people on whether or not this allocation mechanism would cover the general case.

22

Galler: I think this relates to a common mathematical experience; when you cannot prove a theorem very often the sticking-point is the source of the counter-example.

Llewellyn: How often in practice do these obscure errors turn up?

Lucas: Nobody knows how often these cases turn up; that is just the point. A compiler writer is not a prophet of all computer users. Secondly, even if only one user meets the error it causes him a real problem and it causes a full system update mechanism to go into action. Even for only one such case, it is cheaper to make the proof than mend the bug.

Galler: Engineers use the "worst case" approach to testing. Has this been done in software?

Lucas: I can't see any possibility of specifying worst cases in this kind of field.

Reynolds: The difficulty with obscure bugs is that the probability of their appearance increases with time and with wider use.

Dijkstra described some basic features of his work over the past several years on the problem of making programming an order of magnitude easier and more effective. This is described in full in his working paper in section 7.4.

Perlis: Do you think the methods you have developed can be taught to those programmers who have always coded first and justified last?

Dijkstra: No one survives early education without some scars. In my experience, the intellectually degrading influence of some educational processes is a serious bar to clear thinking. It is a requirement for my applicants that they have no knowledge of FORTRAN. I am not being facetious. 
Falkoff: How do you describe a set of computations precisely and completely without using a program in some language?

Dijkstra: The most basic aspects are, on the one hand, what a routine does for you and, on the other hand, how it works. My point is that on no level of abstraction are both of these aspects simultaneously of relevance if the routine is not recursive. These aspects can therefore be clearly separated.

Bauer: I agree entirely with Dijkstra; some current languages are doing considerable harm in the educational process; one can't help mentioning PL/1 here.

Randell: And ALGOL 68.

Bemer: I agree with Dijkstra on the value of elegance and simplicity in one's thought processes and in programming. I agree also on the difficulty of retraining people out of old habits and I wonder what percentage of the total programming population he would like to retrain?

Dijkstra: One hundred and ten per cent!

\section{3}

Lang: You describe some intellectual tools which you say have increased your own programming efficiency about five times. Have you been able to transmit these to your colleagues with a similar effect?

Dijkstra: Yes.

\subsubsection{Debugging}

This important topic occurred many times in discussion during the week The working paper by Teitelman (given in section 7.14) is particularly relevant and much discussion centred round the work; in particular, on the relative advantages of on-line versus off-line debugging and on the distinction between debugging within individual modules and testing the total status of large systems. The latter distinction was expressed by Aron.

Aron: We are discussing two different things. One is debugging: the term we use for an individual finding errors in his own work within the design he created. The other is testing: the term applies to identifying and resolving problems that arise in the system as a whole rather than in individual components. Testing is done by groups, as an individual programmer lacks the total knowledge needed to test large sections of the system. The emphasis on testing should never be on individual modules and as long as system testing is confused with debugging we can expect test plans to be incomplete.

The paper by Needham given in section 7.10 is also relevant to the subject. Comment which, in the editors'opinion, relates specifically to that area defined by Aron as "testing" will be found in section 5.

\subsubsection{On classical techniques}

Brown: There are two classical techniques: the snapshot during execution and the core dump after the program has died.

Hopkins: Programmers call their errors "bugs" to preserve their sanity; that number of "mistakes" would not be psychologically acceptable!

I think that the higher level the language used in programming the better. This was clearly understood by Shakespeare who was against assembly language coding:

"Bloody instructions which being learned, return to plague the inventor" (Macbeth).

Programming languages should have structure. The user must be helped to write in a proper style which will improve the standard of the resulting program. Machines don't have side effects; neither should languages.

Dijkstra: I would like to applaud Hopkins' remarks! 
Oestreicher: One has to remember the need to test exhaustively all conditions which might arise; things which seem locally impossible may well happen due to global effects. Testing for impossible errors is a fact of life.

Lampson: There is sufficient know-how available by now to make it possible to design a machine that would make the writing of reliable programs easier. Does anyone know of a manufacturer seriously doing this?

24

Mention was made of work by John Iliffe and of Burroughs equipment. An entirely different, but equally 'classical', technique was described by Burkinshaw.

Burkinshaw: Get some intelligent ignoramus to read through your documentation and try the system; he will find many "holes" where essential information has been omitted. Unfortunately intelligent people don't stay ignorant too long, so ignorance becomes a rather precious resource. Suitable late entrants to the project are sometimes useful here.

\subsubsection{On on-line and off-line debugging techniques}

Wirth: I would like to discuss the trend towards conversationality in our tools. There has been, since the advance of timesharing and on-line consoles, a very hectic trend towards development of systems which allow the interactive development of programs. Now this is certainly nice in a way, but it has its dangers, and I am particularly wary of them because this conversational usage has not only gained acceptance among software engineers but also in universities where students are trained in programming. My worry is that the facility of quick response leads to sloppy working habits and, since the students are going to be our future software engineers, this might be rather detrimental to the field as a whole.

Perlis: I hold the opposite belief: that conversational techniques aid and abet systems programming and improve the programmer. Better use can be made of the support software used in systems work with full interactive contact; and system programmers require to be on-line. By its very nature, programming is done by development. Languages like ALGOL don't allow this; the program modules have each to be written in one fell swoop. People don't think like that; conversational systems permit programmers to work from the top down and to compute even with incomplete specifications.

Galler: Electric toothbrushes provide an interesting analogy. Like conversational systems they were regarded with great suspicion when new. However, it turns out that people using them have less dental caries just because the electric toothbrush is a better tool.

Teitelman: I agree with Perlis. My own experience with conversational systems is that rather than leading to sloppier use, if anything they promote a more efficient, systematic approach to debugging and testing. The fast response encourages users to checkout their programs thoroughly, e.g. run the program again after making a fix to see if the symptoms really go away. On the other hand, when system response is slow due to overloading, users get frustrated and try to second-guess their programs and then they get sloppy.

Ross: If you are worried about sloppiness on-line, make your users pay real money; this helps!

Needham: In dealing with systems of such complexity that the man fixing a bug doesn't understand too much of the system, you must avoid making it too easy. If it is too easy to walk up to a console and put in a fix you run the risk that it will be locally right but globally wrong.

\section{5}

\subsubsection{On automatic analysis methods}

Lowry: I will start with a strong statement of opinion. I think that ANY significant advance in the programming art is sure to involve very extensive automated analyses of programs.

- If you want better debugging of programs, then the computer must analyze the programs and find the bugs. 
- If you want efficient code then the computer must analyze programs and restructure them from a simple flexible form to a highly stressed efficient form.

- If you want briefer, more natural programming languages then the computer must analyze the programs to provide accurate contextual interpretations of the new forms or expressions.

- If you want better documentation then the computer must analyze the programs to produce abstract representations of the programs from a variety of points of view.

- If you want programs which adapt easily to changing environments then the computer must analyze the programs to perform the adaptations - perhaps working from separate environmental descriptions-so that old code may be more effectively reused.

- If you want to decompile (or decouple) code to a less committed form then the computer must analyze the code very thoroughly (or psychoanalyze it as Perlis suggests).

- If you want more informal conversational computing then the computer must analyze the code to insert implicit constructs and recognize and query irregularities.

Now I know that some of you are currently developing or applying techniques for analyzing program structure-but I think it is mostly nibbling at the periphery. To those others who are trying to make a significant contribution to the programming art in a way that does not involve extensive automated analyses of programs, I would suggest that you perhaps climb a tree—or seek some other solitude—and consider whether you have a chance.

Doing thorough analyses of programs is a big job. I don't think you can just diddle at it. It requires that you use a programming language which is susceptible to analysis. I think other programming languages will head either for the junk pile or to the repair shop for an overhaul, or they will not be effective tools for the production of large programs.

The main point I wish to make is that the analyses required to obtain the benefits I described are closely related and the more of these benefits we attempt to obtain in a single context, the more effectively all of them can be accomplished.

Perlis: What properties of language are important in the context of the need for thorough automatic analysis of programs? It seems to me that some languages may not be susceptible to analysis; can you give some examples?

Lowry: Assembler languages are very intractable. APL and PL/1 have some difficulties in flow analysis but these can be cured fairly easily. FORTRAN is easier to analyse.

Teitelman: However clever the analysis program is it doesn't know what the user really wants. One needs a snapshot of the right words and the only way to do this is by on-line debugging with the programmer present.

26

\subsection{Performance measurement and improvement}

The subject of software performance was covered in some detail at Garmisch and so relatively little material was presented at this conference. Papers were presented on a summary of system evaluation tools (by Gotlieb and McEwan) and on 1108 hardware instrumentation (by MacGowan). These are given in sections 7.6 and 7.9 respectively; for editorial reasons, the latter is given in part only.

Bauer: On a large dual processor system we studied recently we used hardware monitors for two purposes. One was to see what the hardware was doing and as a result we could reconfigure the hardware quite extensively. This gave us better performance and we could send much of the hardware back to the manufacturers. An additional benefit we found later was that the application programmers could make good use of the detailed hardware traces. They found it easy to correlate the traces with different parts of their programs; they then redesigned their programs and improved performance quite considerably. 
Schorr: An interesting thing to try is using measurements on existing systems to extrapolate to new systems. This turns out to be very difficult. One problem is that programmers adapt readily to the machinery available and it is hard to tell whether you are measuring the characteristics of the hardware or of the user's adaptability.

Teitelman: Timesharing systems need to provide aids to measurement for user programs. Obvious things are access to a real time clock and a computation time clock so the user can hand tune his program. Our system has several facilities like this which are widely used.

Ross: Firstly, every computer should have a finely divided clock which is accessible to programs. Secondly, in the early days one had audio signals and store displays with which humans could recognize patterns in many ways and derive a great deal of valuable run time information. We still need such tools. Thirdly, is it possible to categorize in a handbook the useful things one needs to measure?

Galler: The ACM SIGOPS group is working on this problem.

Aron: One purpose of measurement is to tune up a program you expect to run often enough to warrant the expense of tuning. The second purpose is to evaluate a changing configuration. This is much harder to do; a designer faces a complex topological problem in trying to estimate the effectiveness of a range of configurations which he is considering. It is difficult at present to see what measurements would most assist.

Needham: Systematic techniques for measurement are only appropriate in some cases, such as when a system is doing a fixed job. It doesn't help in the changing behaviour patterns of the average big job shop.

Gotlieb: I disagree; I think that even in a university shop these user patterns have more consistency than you might think. This is exactly why we want measurement: to resolve such differences of opinion!

Galler: We have taken many statistics recently and find our users' 27 behaviour surprisingly consistent. The statistics are shown to the users and are very helpful in enabling them to get more out of the system. Whatever is useful to the systems programmer should be made available, in general, to the user who needs similar tools.

Randell: Unless your multi-access system is large it is debatable whether there is enough statistical averaging for the measurements to be useful. There is a different problem: is an individual user to be allowed to improve his own utilization to the direct detriment of others? Preferably not! Avoiding this situation should be a design aim and until it is achieved maybe he is better off without the tools which would allow the problem to break out.

Ercoli: Computers and software are service systems of much complexity. Average measurements are difficult but the analytical tools are both inadequate for the study of this kind of system and often not familiar to software engineers.

Sharp: Scheduling multiprogramming systems is one of the least understood areas in the subject; with machines like ILLIAC IV coming along the situation will get worse. This alone seems to me to justify measurement; without some statistical evidence one cannot design. How, for instance, do IBM do it?

Schorr: Statistics are usually so biased by local conditions that in practice they are seldom of much real use. 


\section{SOFTWARE FLEXIBILITY}

The material under this heading has been classified under two somewhat arbitrary subheadings: Portability and Adaptability.

Adaptability is concerned with enabling a given system to grow and change, as discussed for example by Warshall. A related problem is that of moving an existing large system without taking it out of service, as discussed by Hopkins. Portability is the more restricted problem area of moving a specific system from one environment to another.

\subsection{Portability}

\subsubsection{Definitions of the problem area}

Perlis: Portability is the property of a system which permits it to be mapped from one environment to a different environment; adaptability concerns the alteration of a system to fit the needs of a user without necessarily changing it from one machine to another.

On the definition of the terms "system" and "environment" I should like to make the following comment. There is a layering, like an onion, that cascades from out to in. The interface between any two layers is always characterized by a language and indeed if one does not have a language one does not have an interface. A system is constructed on an inner environment and prescribes an outer environment for the user.

Dijkstra: A very tiny both linguistic and educational remark concerning terminology we use. Please don't use the term "onion shaped," and I'll tell you exactly why. Because you're talking about something that is essentially a linear layer. When I was at MIT I heard people talking about protection rings and it turned out that as soon as you started talking about it they had to jump to the blackboard and make rings using a two dimensional representation for an essentially one dimensional hierarchy. If you now introduce the term "onion" you'll appeal to a three dimensional picture of essentially a one dimensional structure and it might clutter up your thinking, because there might be another dimension to be added to it. So please, no onion.

\subsubsection{Description of techniques}

Perlis: Technically we are concerned with the investigation and production of support on new machines sufficient to accept systems transported from old machines. Systems need support and should be adjustable and tunable if the new support turns out to be not too good.

The examples presented to illustrate portable systems were discussed under these headings, classified by the type of support to be provided in the new environment.

\section{9}

\subsubsection{High level language support}

This assumes a common high level language between the old and new machines. See the working paper by W.S. Brown on the ALTRAN system in section 73.

Reynolds: A language can be unsuitable in two ways; if it is not sufficiently concise then that can be overcome by macros but if it does not effectively allow you to write the program or represent the data at all then macros will not get you out of trouble. 
Brown: Macros are used to overcome the inability to express target machine characteristics in a FORTRAN program. Limitations with regard to data representation are got round by using primitives.

Perlis: The representation of any kind of data as integers in an array can be done if you have enough primitives around.

\subsubsection{Bootstrap from the old system}

Ross described the AED system developed by the MIT Computer Aided Design Project over the last several years. We summarize the presentation here.

Ross: AED-0 is based on ALGOL 60 with data structures and other extensions. Its compiler is mainly written in itself (95 per cent) and was designed with portability as one of the aims.Portability can never be both complete and efficient and it is therefore best to tune up critical modules after the move to get an efficient result. This tuning can to some extent be automated. The transfer is carried out by a complex bootstrapping approach including a stage of "half-bootstrapping" in which code for the new machine is generated on the old one.

Strachey: Martin Richards' BCPL was transferred from MIT to the KDF9 at Oxford as two decks of cards and some scruffy notes. It was got running in about a week. The entire compiler is in BCPL and is designed to be machine independent. The support system, including I/O is in assembly code. The code generator was rewritten in ALGOL to provide an initial means of generating code.

\subsubsection{Summary of techniques}

Feldman: When one goes from one machine to another there'll be a large set of environmental specifications: the size of a word and the kinds of direct access, peripheral and tape storage and various other things of this sort. This caused Stan Brown, for example, a reasonable amount of difficulty in parameterizing his system in such a way that all the pertinent parameters could be incorporated. Another thing is the transportability of data. Now, of course, with a small piece of data that isn't a large problem. If, however, there are large elaborate data files that are segmented in such a way as to be compatible with certain strategies because of certain direct access devices and one goes to another system in which the devices are entirely different and one has to entirely restructure the files, then that is a very large problem. The data itself must be entirely restructured.

Two other points. Firstly, once a program has been moved it becomes part 30 of the support for the other programs to be moved. Secondly, not only does a portable system have to have its support specified but it is very important that the program that is about to be moved is written in such a way that it can tolerate less than perfect support. If one expects a certain exact support in order to move a program, one is going to be hard pressed. The program itself should be modifiable to make up for imperfections in the support of the target system. Furthermore, this flexibility will always be important in the second process, which is getting a system to run well. Assuming that one has got it running somehow, you can always get it to run much better by making changes in the way it goes about its business. So this flexibility in the way the program itself is written is necessary in order to move it in case the support is not perfect. This is what happened with the BCPL example; there was an ALGOL compiler on the target machine and BCPL was written in a sufficiently transparent way for the code generating rules to be written in ALGOL and put on the machine quickly. The text that was implemented was not identical to the text that was sent.

\subsubsection{On portability of data}

Bemer: (from "Straightening out programming languages") "John Haanstra has said that compatibility is not a goal, but rather a property which enables the goal of data and program portability to be achieved. I have my own lemma that 'if the data is not transferable, the program cannot be transferable'. It is quite evident now that the separate divisions of COBOL facilitate program portability. However, we can and must do more for COBOL along this line and, more importantly, carry it to the other programming languages, both procedure and problem oriented. Let us concentrate on the distinction that information can be obtained only when one knows the 
conventions of data representation. This brings to mind a curious sequence of events, actually, a cycle. The name CODASYL (the coinage of which was my small contribution) incorporates 'data'. When we started the standardizing bodies, we got a little fancier and said 'Computers and Information Processing'. With the marriage to communication and data bases, the plain facts are that we will process data and, incidentally, some information. Computer based systems can move data around from place to place, put it away, find it again on the basis of its packaging, and (as in the case of cryptography, for example) perform transformation upon the data, all of those absolutely independent of the information content.' Data is our raw material. Software and hardware are only tools for manipulation. In some way the higher level languages, in the vacuum of not knowing enough about data structure, have achieved a disproportionate importance and a warped direction; one direction per language, in fact. Indeed, if I have a process to perform upon data, I may choose one of several information procedure languages. Conversely, more than one user of the same data should be allowed to operate upon that data by various information procedure languages."

\subsubsection{On design for portability}

Perlis: In a system such as Brown's, the assembler and linking loader are as important as the FORTRAN compiler. I suggest some principles to characterize the design of portable systems:

1 Every output can be an input. (Closure)

2 There is a set of properties that one can define to characterize a "block", and the coupling between blocks.

31

3 Then blocks may be gathered together so as to produce a new collection more intensely coupled than the originals.

4 This gathering process is subject to ever finer decomposition, i.e., it may operate within blocks, and interruption in the sense that it is a sequential process with memory-prior gatherings may be utilized in subsequent gatherings.

$5 \quad$ Naturally all gatherings may be named (identified).

6 Furthermore there is a set of properties called parameterization rules, which permit weak coupling ("coupling at a distance").

Then the problem is:

1 How to characterize blocks.

2 How to characterize permitted coupling.

3 How to characterize degrees of coupling, including parameterization rules.

4 How to characterize the sequential machine (control, operations and memory) which accomplishes the "gathering" task. A new component, scheduling and resource allocation, is introduced when segmenting and parallel execution is possible. This is the new dimension introduced within operating systems.

One final point is that it is well worth looking at the work on "Generalized Programming" by Holt and Turanski.

Dijkstra: (from "Structured Programming") "Any large program will exist during its lifetime in a multitude of different versions, so that in composing a large program we are not so much concerned with a single program, but with a whole family of related programs, containing alternative programs for the same job and/or similar programs for similar jobs. A program therefore should be conceived and understood as a member of a family; it should be so structured out of components that various members of this family, sharing components, do not only share the correctness demonstration of the shared components but also of the shared substructure."

\subsubsection{On writing portable software}

Lampson: What can we do to help the man writing portable software not to make mistakes? 
Ross: Educate him. (Laughter) There is no other way. We should note that a simple system can indeed be transferred in a few days but bootstrapping a complex system cannot be made simple.

Lampson: The major problem is avoiding doing things that are machine dependent; for example, on word length. It does not seem unreasonable to hope that in the future one might have languages that will discourage one from writing this sort of thing and compilers that will detect when you have done it.

Brown: We have two tools of great value in writing portable software. One is a compiler which enforces the use of our subset of FORTRAN. The other is the mere existence of the macro facilities, which encourage the writing of clean code - by this I mean avoiding things like equivalence through mismatched COMMON statements.

Ross: A new word length is not a problem; utilizing new resources in a complex system is a major task.

32

Perlis: It seems to me that the first solution that one can see to this question of not making mistakes (premature bindings) is the recognition of a class of functions where only one function existed before. For example: a system programmer develops a system for a computer with one register, the accumulator, and wishes to map the system on to something like the PDP10 or the IBM S/360 where there are multiple registers, without losing efficiency. Clearly he should have designed the system so that the number of registers utilized was a variable; so that the number of registers of a particular machine may be a parameter when the system is mapped on to the machine. Now if he doesn't allow for the possibility that a single-register machine is a special case of n-register machines then portability is going to require a major rewrite of the system. How you anticipate possible machine designs, the logical design of machine systems, nobody I think can really say. So I think that you're always going to have to backtrack when transporting a system, but in backtracking the way to do it is to recognize that, in situations in which you have (say) two data points instead of one, the value "two" is still only a special case. Some of the things are obvious, such as machine core size, because we know that all machines don't have the same number of machine words. The number of registers is now obvious. I guess people are now saying that parallelism is obvious but take a machine like the ILLIAC IV where you have an arbitrary number of accumulators all of which work in parallel. In this case we're not talking just about (small) parallelism in data transfers. How could someone building a system in, say 1960, anticipate that the ILLIAC IVs might be setting the tone for a whole new line of machines in the 1970s? So I think that there's probably going to be a limited time range when we won't be making silly mistakes that make portability difficult. Beyond that limited time range we'll find we've made some disastrous mistakes.

Strachey: I want to come back to this business of how can we help people to make systems, write systems, in such a way that they can be transportable. It's not entirely a question of not putting in features that we haven't thought of - not putting in the right number of registers or something like that. I think one of the chief difficulties is that the general standard of programming is extremely low. Now this is a thing which I know Dijkstra's been talking about and this is actually what Doug Ross was saying. I think that I would like to suggest again, that the general standards of programming and the way in which people are taught to program is abominable. They are over and over again taught to make puns; to do shifts instead of multiplying when they mean multiplying; to multiply when they mean shifts; to confuse bit patterns and numbers and generally to say one thing when they actually mean something quite different. Now this is the standard way of writing a program and they take great pleasure in doing so- "isn't it wonderful? It saves a quarter of a microsecond somewhere every other month" Now I think we will not get a proper standard of programming, we will not make it possible to have a subject of software engineering until we can have some proper professional standards about how to write programs; and this has to be done by teaching people right at the beginning how to write programs properly. I don't know that we know how to do this yet but I think we should make a very serious effort to do so and I think one of the things we ought to do perhaps at this conference is to make a great point about trying to improve the standards of programming in general. I'm sure that one of the first things to do about this is to say what you mean and not to say something quite different.

\section{3}

Perlis: I think it is inevitable that people program, and will continue to program, poorly. Training will not substantially improve matters. Using subsets of language doesn't help because people always step outside the subset. We have to learn to live with it. 


\subsubsection{On effectiveness of portability in practice}

Brown: A sufficiently radical change of hardware, e.g. the move to highly parallel multiprocessing, may render the algorithms themselves obsolete so that even if portability is achieved, the new hardware may be used hopelessly inefficiently.

Feldman: If you are using a system very hard and pushing it to its limits, you cannot expect that system to be portable.

Poole: It seems to me that inefficient software on a machine is better than none at all.

Feldman: (from "Towards automatic programming") "The idea of extending a language through macro expansion is appealing and is certainly useful for minor syntactic changes. The problem is that to add a significantly new construct to a language with reasonable efficiency, one must change the compiler in nontrivial ways. The solution is to write compilers so that they may be altered without undue pain. Several translator writing systems make this possible. Alternatively one could write a clean compiler in a base language and extend the compiler when needed. Our understanding of compiler writing is reaching the point where it should be possible to design extensible compilers. This is a topic that deserves considerably more attention."

In connection with this topic, see the paper by Schorr, reproduced in section 7.12.

\subsubsection{On transfer of know-how}

Ross: We have never yet set foot in a user's premises to help put the AED system in. Furthermore, users are able to write systems they can bootstrap to new machines with little help from us. But knowing how to achieve a full bootstrap of the AED system itself is quite another thing. We can't tell people how to do that yet.

Brown: We hope to solve this problem by putting in a lot of effort on documentation and system support software.

Buxton: I want to make one or two slightly cynical remarks on the subject of portability.

Since I've recently left the ivory towers of industry and returned to the sordid commercial life of running a university department, I feel a little cynical as a result. My impression is that we've been treated to an estimable and very interesting review of good, solid, working, successful techniques for transporting software around the place. On looking back, it occurs to me that some of these techniques really have been around for years-I can recall, no doubt most of us can, early bootstrapping between machines being done in the late 'fifties. An interesting question, which occurs to me then is to wonder: why isn't portable software with us? So I ask myself the 34 following questions. Firstly: who wants it? The answer is the users. Secondly: who has to produce it? The answer is the system designers - it seems very sensible to say that if you really want portable software you must design your software system with that aim in view from the ground upwards. Now, why is this not done? The answer here is, I suggest, that the system designers by and large reside in the manufacturers who actually produce the vast majority of software used by the average user. This software is not portable because the manufacturers do not regard it as in their best interests to have portable software. This is why we haven't got any. The portable software we have is, in the main, produced by users who are also systems designers, working in mainly academic environments. The only places where it tends to go into general commercial use is in fact in the bureau business. The business of a manufacturer is to get his clients and then to keep them and this he does by a process known as "Free competition in the open market". This is implemented by taking the clients he's managed to get and then totally insulating them from all other possible external influences. This is done by a process known as education. If this process is successful then with any luck his clients will see only his future range of machines. Portable software would be a disaster because they might be able to take useful application programs elsewhere. What a manufacturer might conceivably want is software that is portable on to his new range of machines, when he's thought of it, and only on to that new range, not any other machines. 


\subsection{Adaptability}

One of the major contributions on the subject of adaptability was given in a talk by Warshall, who made reference to a report entitled "The Representation of Algorithms" available from Applied Data Research, 450 Seventh Avenue, New York, N.Y. 10001, U.S.A.

Warshall: When discussing portability we saw three examples that I regard as sensitive and competent applications of a conventional technology for moving programs. I would like to talk about the philosophical picture of portability that underlies that conventional technology. I claim that technology rests very critically upon the fact that the targets between which portability is occurring are very similar in structure. In any of these systems or in any system which facilitates transport, what is being transported is, ultimately, descriptions by people of computational processes. People describe problems in some kind of language. It is a characteristic of languages which describe computational processes that they are a mixture of two things. First of all they implicitly define the mapping which you are trying to perform by executing the process. Second of all they define a certain intended allocation of resource to accomplish that mapping. If you are dealing with two more or less similar machines as targets for translation of your language, the trick throughout the conventional technology is to attempt to strip out of the linguistic form exactly those representational commitments which have to do with the differences between the two machines. If, for example, one machine has an instruction which does floating-point multiply and another machine does not, you raise the language level and you do not permit the language you write in to dispose in detail of this level of resource. The language speaks about "floating-multiply" and the translation is different in the two systems; in one case it is to a unitary operation and in the other to some kind of subroutine call.

To every part of the expression of the mathematical process in the "higher level language", there corresponds a structure downstairs in each of the targets. It is true of the conventional technology that the two programs in the two targets always look very similar to each other for that reason. They both reflect the structure of the program description in the higher language, and by higher level here, I do not mean more closely connected with the problem: I mean containing fewer details of resource commitment.

What happens when the target machines or the target environments become very dissimilar? Then two programs to express an identical mathematical function, for instance, on the two targets would look quite different from each other. For example, if you imagine an information retrieval system on a machine which has a couple of tapes for secondary storage, then the program will look like an enormous contortion to avoid dead rewind time. Now a program to perform the same function on a machine with random access storage would be written in an entirely different way. In effect, when the target machines for your translation become more and more dissimilar and you have to strip out more and more representational commitments from the linguistic form, you always end up in the same trap. The linguistic form you are working with when you get through is so far away from either of the targets, in that you have made no representational decisions worth talking about, that the software's problem of mapping down is epic in its proportions. For example, you might write all programs as though you had infinite memory and then let some automatic device worry entirely about storage strategy and resource strategy. That's certainly a harder job for the software than the job it has now.

The only way that we know out of this trap is to take the two dissimilar equipments and make them look similar. That is to say, we introduce a layer of software on top of either of the machines or both of them and regard these simplified machines, defined by the interposing software, as the real machines we are mapping to. They are more similar, the maps become more plausible, but of course you have introduced some degree of inefficiency; inevitably, because you are now regarding as atomic units things that are really combinations of atomic units, and solutions that involve breaking them up are no longer available to you. I claim we are already in the situation of dealing with targets that are in fact so dissimilar that a conventional approach to transport forces us to choose between an absurdly difficult compiling problem on the one hand and impossible inefficiency on the other. I think that is the reason why the conventional approach has shown no fruit at all when dealing with, for example the use of secondary storage as a resource.

\section{NATO SOFTWARE ENGINEERING CONFERENCE 1969}


I would like to say next that I am not claiming that you will ever reach a situation where the two target machines are so dissimilar there exists no language "high enough" to permit mapping down to both of them. I am merely asserting that if that language ends up looking like a Gödel numbering system for programs, I am not sure it is a useful logical construct for us, or even for mathematicians. Incidentally I would like also to say that it is not clear that such a "higher level language", if the target machines are sufficiently dissimilar, has very much to do with a suitable language for human beings to write in. "Higher level" here really means "less committed representationally" and has nothing to do with closeness to natural expression of problems. This is a confusion of issues which I think is found throughout our literature.

Having said that there are intrinsic limitations in conventional methodology, one might ask whether there is any alternative. I claim there is an alternative, although I certainly can't prove it is the right one, and I 36 would like to give you an example of what I mean. This example comes from what may superficially seem a strange place: code optimization. Imagine that you are attempting to compile a program in, say FORTRAN, for a machine that does not look like the abstraction: the FORTRAN machine, and that in fact looks seriously dissimilar to that machine in a very crucial way. In particular the target of the compilation might be a machine that is not a sequential synchronized processor. Clearly, your program for the FORTRAN machine contains representational commitments about sequence and synchrony which are just plain wrong for the target. A group of people, friends of mine, have been working for some time on this problem. This group found that conventional techniques simply would not work. By conventional techniques I mean starting with the FORTRAN program as the highest level language dealt with and essentially compiling down in successive stages, each of which was more representationally committed than the previous stage. They found that the fruitful way to look at the problem was as an instance of the portability problem, i.e. to envisage it as an effort to transport from the FORTRAN machine to an asynchronous processor. What they did in effect was not to map down but to map up, i.e. perform a process which they themselves call "decompilation". Having isolated the most crucial representational commitment that was getting in their way, namely the commitment having to do with sequential synchronous processing, they developed automatic techniques for methodically transforming a program in the FORTRAN language to something at a "higher level". This does not mean something closer to the problem or closer to a human being's understanding of the problem, but merely something with fewer representational commitments. They convert the FORTRAN program into what might be regarded as an asynchronous automaton which happens still to have some structural features that came from the initial organization of the FORTRAN program. This expression in the form of an asynchronous process has the additional virtue of being deformable; that is to say, by formal techniques it is possible to alter a process with a different degree of asynchrony. In essence, instead of finding a language that a man can write in and that is "high enough" and then mapping down in all possible directions, they started with something in which a man could write that was not high enough, they mapped up, then across and then back down again.

I would submit that given our notable lack of progress in applying a current technology to situations in which the targets are seriously dissimilar and given the kind of remarks I made earlier about why you might expect this to be so, it might be an extremely worthwhile thing to break out of the mental trap of assuming that the highest level language in the system has something to do with a problem oriented language. We should consider the use of software as a device for eliminating representational commitments; maybe trying that strategy for a while will help us in solving this very difficult portability problem where the targets are systems that are quite dissimilar from each other.

Bauer: I don't quite understand what you mean by decompilation. Does this involve more than relaxing the strict ordering sequence of instructions?

Warshall: The representational commitments that derive from the model of the FORTRAN processor as a sequential synchronized device go much deeper than simply the problem of the ordering of instructions. In particular, one of the most crucial things to disintegrate in the source program is the concept of "variable name". The name "A" for example in a 37 program is the name of a class of values no two of which are interesting at the same time; that's why they can share storage and why they can share a name. If you want to explode the process, that is to say take advantage of increasing degrees of asynchrony, it is necessary to explode the variable name to a set of other things, and how many other things is a very subtle function of how the program is constructed. 
Perhaps it would be more informative if I gave you an idea of what kinds of deformation occurred. This group performed amongst other things the following:

1 Elimination of common sub expressions on a global basis, not on a flow block basis.

2 Loop flattening; by loop flattening I mean the following. Imagine that a loop has three computations in a row. In the $\mathrm{i}$ 'th iteration it first computes $\mathrm{A}[\mathrm{i}]$, then using $\mathrm{A}[\mathrm{i}]$ computes $\mathrm{B}[\mathrm{i}]$, then using $\mathrm{B}[\mathrm{i}]$ computes $\mathrm{C}[\mathrm{il}$. The optimizers do tricks like, for example, changing the initialization to make it more extensive so that, instead of starting out with $\mathrm{A}[0], \mathrm{B}[0]$ and $\mathrm{C}[0]$, you start out with $\mathrm{A}[2], \mathrm{B}[1]$ and $\mathrm{C}[0]$ and then the three lines of computation can march along in parallel.

3 Decision postponement; it is sometimes more economical not to decide which of two things to do but to do both of them at the same time before deciding which result to use.

4 Arbitrary degrees of internal buffering to interface parts of the process; for example, if you have a bunch of decisions, you have iterative decision making in one place while the results are being developed somewhere else. These two things can get arbitrarily out of phase with each other.

5 Most important of all, full pipelining at all levels. This means that if, for example, you have a machine which is operating on inputs and producing outputs, it is not necessary for the machine to wait until an output is produced before the next input starts being processed. Pipelining is used here in the same sense in which it is used in hardware in systems like the CDC 7600.

We conceive of a program as something that can, with the proper equipment, be solving more than one problem at a time and we have to provide the machinery for getting the right answer associated with the right question as we get through. Now, it is clear that this kind of explosion of a process is a good deal more extensive than the partial ordering of FORTRAN statements.

Schorr: The process of going up to a higher representation, then across and down again sounds like a nontrivial job. I wonder whether, in the case of FORTRAN for example, the cost might not in some sense be lowered by perhaps providing a "sift" from one FORTRAN to another followed by a correction process?

Warshall: One possible realization of this approach that we have considered is the idea of what we call the "FORTRAN laundry", based on our knowledge of the way in which two different manufacturers' compilers work. We take FORTRAN written for one machine and translate it to FORTRAN written for the other machine.

The more important and crucial thing to get across, however, is that I think there are three essentially different techniques that I am talking about. One- the conventional—is to have the language in which the programmer writes be the highest language, i.e. the least representationally committed language, in the system, and to restrict the software to mapping 38 down. The second is to decompile, i.e. to eliminate some class of representational commitments, and deform, which is not the same as compiling down. You go across and then down. The third is to go all the way up to something so decommitted that you can sensibly compile down from it towards either target.

The remarks I made at the beginning suggest that we have reached the limits of the first technique and that perhaps the second will prove more fruitful than the third; it is the second that we are in fact involved with. The decompilation process is certainly extremely laborious, complicated and space consuming and unless you are really concerned with optimization would not recommend using it. However, I think the application of this technology to other situations like, for example, the allocation of secondary storage, could have very fruitful results.

Bauer: Adaptability means the modifying of programs to relate them to specific users. This is of particular interest to those with large application programs. Aron pointed out the difficulty of getting a good initial specification and the need for continual changes at late stages in the design process in ways which are neither predictable nor even reasonable.

Where you are concerned with a large program and a large data base, the data structure must be included in the system design consideration, otherwise you are lost before you start. The logic of the data structure is tied into the logic of the program. Two extreme methods of including the data structure in the system design are:

1 Attach a full description of the data structure to the data base.

\section{NATO SOFTWARE ENGINEERING CONFERENCE 1969}


2 Represent the structure of the data entirely in the program logic. Neither extreme is particularly successful. What do we do about this problem?

See also "On portability of data" in section 4.1.4.

Lampson: (from "On Reliable and Extendable Operating Systems") "If a system is to evolve to meet changing requirements, and if it is to be flexible enough to permit modularization without serious loss of efficiency, it must have a basic structure that allows extensions not only from a basic system but also from some complex configuration that has been reached by several prior stages of evolution. In other words, the extension process must not exhaust the facilities required for further extensions. The system must be completely open-ended, so that additional machinery can be attached at any point.

Secondly, the relations between a module and its environment must be freely redefinable. This means that any module, provided its references to the outside world are documented, can be run inside an envelope which intercepts all of these references and reinterprets them at will. In order to ensure that external references are documented, it must be normal and indeed compulsory practice to specify each module's environment precisely and independently of the module's internal structure.

Thirdly, it must be possible to introduce several layers of re-interpretation around a module economically without forcing all of its external references to suffer reinterpretation by each layer. In other words, a capability for extension by exception is required. Furthermore, the system's basic mechanisms for naming and for calls must permit a number of higher level modules to utilize other modules, rather than forcing each new module to create and maintain its own inventory of objects.

To summarize, a usefully extendable system must be open-ended, must 39 allow each module to be isolated in an envelope and must encourage economical reuse of existing constructs. Such a system has some chance of providing a satisfactory toolkit for others to use."

Finally, we include this description of the way in which a system was transferred, while continuing to provide service.

Hopkins: I would like to comment on how to improve a given system to make it portable from a real, live, and successful example. My example is the American Airlines' SABRE system which in 1965 was handling about 2200 inputs a minute on a modified 7090. Their projection from the growth of the airline business was that by 1970 it would be handling 3000 inputs a minute. I don't think many of us have on-line real time systems that have to perform that way; this is a real example of software engineering. The problem was that the 7090 was completely saturated in 1965 and the system consisted of over 300,000 assembly language instructions. In its favour, the system was highly modular and had such things as standard communications areas, standard names, all the programs used copy facilities from libraries and it had supervisor system linkages which were all standardized. Now consider what the problem really was-they couldn't just pull the plug out of that system some day and stop selling tickets—airlines get very excited if they can't sell tickets for a week or more. The system itself had reliability problems which occur in any system where you've got 300,000 instructions. It was a growing system, in that the business changed and so the program had to change; this all meant that the system went down 3 or 4 times a day. They introduced new errors into the system 2 or 3 times a week; sometimes they did not flush them out for a month or more and they learnt to live with this.

Now imagine trying to take this system off a 7090 and get it on to a 360/65; this was a sort of nightmare and the solution was interesting. First of all, they attacked the problem in one very practical way. If you have a really bad problem, you spend all you can on hardware. They bought a system hookup consisting basically of a pair of 7090s, which have communication lines going into them, inputting into a pair of 360/65s. I believe they have twelve 2314 disc units, and they have a whole raft of other secondary storage units.

The short term solution was to simply take the 360 and put a small control program in it and let it watch the 7090s' I/O request queues and do all the drum I/O and the LCS transfers on the 360 large capacity core storage. This gave them about a 20 per cent improvement on the old 7090 system and they were able to get over the next Christmas rush. The long term solution was to bring up OS/360 on the 65 and write a control program on top 
of this which essentially duplicated the same functions as the 7090 control program. Then what they did was to gradually transfer modules over from the 7090 to the 65 .

They were extremely upset about having written 300,000 lines of assembly language code the first time around and they were determined to write in a higher level language the second time. There was no suitable language which was available, which could interface with their systems so they wrote a new compiler, which was called SABRE PL/1, for a PL/1 dialect with low level features. It provides, and this is very important, a mechanism for having non-standard interfaces. In this kind of environment it is very difficult to persuade programmers who are designing the control program and the systems services not to design non-standard interfaces. If you have the prospect of 3,000 inputs a minute and your system has always been just $\mathbf{4 0}$ one step ahead of the sheriff, you are not going to tell people that a microsecond isn't important; too many times they have had to rewrite code to save a microsecond or two. However, they are willing to live with a very few assembly language modules because they recognize the fact that most of the work is concentrated in a very few modules. The higher level language is machine independent in the sense that it knows nothing about the internal registers of the machine; however, it does know how to write nonstandard calls to systems services and it is machine dependent in the sense that it knows that a byte has 8 bits on a 360 . The performance is very good with no global optimization. This is because, as has been pointed out elsewhere, system programs really don't have very many loops and it doesn't pay very much to move things out of loops because the loops just aren't particularly important.

I venture to guess that one of the most important things in gaining acceptance of the product was the fact that it produced an assembly language type listing which showed after each statement exactly how much code was generated. As a sales gimmick, people could see that the compiler was producing clever code in certain instances and this pleased the assembly language programming types. It also proved to be a tool which enabled people to see when code being generated was disastrous; they could see it and make adjustments.

I think, in conclusion, that I'd like to point out that while I treat this as an eminently satisfactory exercise, I shudder to think what will happen if American Airlines have to go to a machine with a different word size or a radically different type of systems services. The machine dependence of the SABRE PL/1 language is rather high in those areas. If you've got this constant fight against time, you're going to have to constantly improve the system. I guess I'd like to conclude also with the fact that at this time I believe approximately half, closer to three quarters, of the system has been recoded in PL/1 and is now functioning on the 360. They are approaching the time when they will be the only people in the world who have a 64k word 7090 as a frontend machine for a $360 / 65$. 


\section{LARGE SYSTEMS}

In this section we have attempted to collect together material that is specifically related to the problems of designing very large systems. By way of introduction, the following comment by Schwartz has been excerpted from the quotation given in full in section 5.1.1.

Schwartz: I must admit that I have frequently asked myself whether these systems require many people because they are large, or whether they are large because they have many people.

\subsection{Case Histories}

Several rather extensive reports were given at the conference summarizing experiences gained in the design of large systems. These reports and the ensuing discussion are the subject of this section.

\subsubsection{A survey}

This section is a transcription of a talk by Schwartz, who also provided a working paper "Analyzing Large Scale System Development" reproduced in section 7.13.

Schwartz: In the early 1950's, a small closely-knit group of skilled programmers working with 256 words of immediate access storage and relatively limited file storage and peripherals, produced a computerized aircraft surveillance system which included a limited set of radar inputs and tracked a few aircraft simultaneously. Based on this successful effort, this small, closely-knit group, in cooperation with the U.S. Air Force, decided to produce a somewhat more complete system. This system would consist of a set of duplex computers covering the entire United States with communication between adjacent sectors, new radar equipment which would permit finer tracking, a number of additional functions, a wide variety of alphanumeric and vector displays and automatic backup between the various sectors as well as within the duplex computers. The capacity of the system was to be considerably larger than the prototype development, and the computer was to have $8 \mathrm{~K}$ words of core storage. Any illusions that the original group had about retaining their past pleasant, intimate, technically exciting working relationship was soon shattered. Within a year approximately 1,000 people were involved with the development of the SAGE System. People were recruited and trained from a variety of walks of life. Street-car conductors, undertakers (with at least one year of training in calculus), school teachers, curtain cleaners and others were hastily assembled, trained in programming for some number of weeks and assigned parts in a very complex organization. The core storage of the computer was expanded to $64 \mathrm{~K}$ words. The originally hoped for capacities of the system were cut considerably. The system was first delivered over a year late at considerably more cost than was originally expected and not too long before people began to realize the 42 threat of the manned bomber was beginning to decrease considerably as long range missiles began to become a reality.

So began the era of the large scale system engineering efforts.

Not too long after this actually reasonably successful start, another rather major effort was begun for an important military function. Those responsible for this one hired and transferred approximately six hundred people within a very short time. After this first step (hiring) was completed, the next problem was to determine what this system was to do. This took, to some people's chagrin, several years. Man's ingenuity being what it is, however, a large number of these people were not wasted. They were put to work on the support (utility) system which would assist the operational programmers when they got around to writing these programs. The support system for SAGE had approximately 1,000,000 instructions and was actually very good, considering the time at which it was built, but it was decided to improve upon the support system in many ways. It didn't make it: about two years later, the support system was not quite ready to handle the operational programs, which were finally being coded. 
In 1961, a small group of highly skilled programmers produced, at MIT, one of the first general purpose time sharing systems, called CTSS. This system evolved into one of the better known and highly successful time sharing systems. Based on this experience, in 1964 ideas began to be formulated for a considerably advanced system called MULTICS. This was an ambitious effort, encompassing a number of new concepts, new hardware, and considerably more system programmers than were required for CTSS. I think it would be fair to state that this system has not proceeded with the same feeling of pleasantness nor satisfaction that accompanied CTSS.

These are a few of many experiences in large-scale system development which have taken place over the past 15 years. Very few of these have brought unanimous cheers from producers and customers because they were on time, met the original specification, stayed within original hardware and remained on budget.

We have heard today a number of reasons as well as solutions for problems in the production of these systems. From many of these experiences, we have learned of techniques and guidelines necessary to approach these massive software undertakings.

Pervading all discussions are several key words, one of these words is control. Control is needed by managers, so that they don't begin a task before the initial specification is clear. Programmers must be controlled, so that they don't invent beyond the requirements which are assigned to them. Control must be exercised over the testing of the system, so that tests for all levels of the system, including the smallest components, have adequate test specifications prior to their production. These tests must then be executed and verified. Control must be exercised over new versions of systems, to make sure that they are at least as good as the previous version, before they are unleashed on the world. Documentation must be controlled but must constantly keep pace with the system development.

Another word is flexibility. Managers must be willing to adapt as situations arise requiring changes in scope or technique. The basic system must, through such means as data description divorced from procedures, centralized data maintenance, and data-independent programming languages, be flexible enough to permit change and evolution without reprogramming. People must be flexible enough to tolerate rather drastic changes in direction.

The third word is people. A wide variety of levels of skills in people are required in this kind of effort. People differ enormously in capability and $\mathbf{4 3}$ working styles. This presents considerable management problems. Better measurement techniques would be very valuable. Some subset of the people must be able to communicate outside of their own sphere, which might be software, documentation, testing, engineering, or administration.

Lastly, there is the word management. To handle large numbers of people, organized in a fairly complex structure, keeping them motivated while under quite stringent control, to understand the mass of details concerned with the production process, to make decisions based on too few or too many inputs of a sometimes highly emotional body of people, to be able to face superiors or customers with occasionally drastic recommendations for change in scope; all of these require the large scale system manager to be skilled, flexible, tolerant, informed, extremely tactful and, unfortunately, rare.

I must admit that I have frequently asked myself whether these systems require many people because they are large, or whether they are large because they have many people. There is little question that they could be done with fewer people than have sometimes been employed, given better initial planning, better key people and a more intelligent starting phase. But it is also true that these systems require many parallel activities outside the development of the central operating program; unrealistic goals and schedules are specified, communication problems exist at many levels. All of these factors and others beside conspire to create much larger organizations than seem theoretically necessary. Thus, although experience speaks against massive efforts, it also indicates they will continue into the future.

\subsubsection{Apollo Programming Support}

This section was transcribed from a talk by Aron which covered, at greater length, information that he gave in a working paper entitled "General Remarks on Apollo Programming Support"

\section{NATO SOFTWARE ENGINEERING CONFERENCE 1969}


Aron: I'd like to discuss the Apollo project, primarily for the purpose of giving a feeling of scale on a particular task which, through its visible results, can be seen to have been a successful project. The Apollo 11 mission, which actually landed men on the moon, was evidently successful because you all saw it. Within that project, which was an immense hardware/software/organizational activity, there was a large software system. In fact there were several large software systems. I'll talk about the ground support of the space mission and primarily about the portion of that which was conducted at Houston, Texas. There were other portions conducted by a number of different manufacturers at Cape Kennedy, at the Manned Space Flight Center, at the Jet Propulsion Laboratories, and at a number of tracking stations round the world; and of course in the space ship itself, where you had computational capability and direct communication with the ground. The significance of the many locations is that you have a much more difficult interface co-ordination problem because you have geographically separate activities that must work in synchronization and must trade information in real time during the mission.

As a first step in giving you a feeling for scale, the Houston, Texas operation by itself ranged over a period of years from 300 individuals to about 600 individuals, and at the largest point there were in the region of 300 programmers. The remaining people were in support or in administration associated with the job. This group started their experience in space activities about 1959 on a project called Vanguard, a Navy satellite project that put a very small satellite in earth orbit. This was then taken over and merged into the American space agency's (NASA's) program, called 44 Mercury, which put men into space, followed by Gemini, which went into the two man space mission, followed by Apollo, which had as its objective the landing on the moon. The initial project required a portion of a 704 to track and maintain trajectory and orbit information on the Vanguard satellite. The system, which was used on Apollo 11, ran on one IBM S/360 model 75, but the support system requires a total of six for its development, so in Houston there are six model 75's. In addition there was a model 50 for program support. All these machines were associated directly with the ground support programming, which excludes communications and activities associated with data reduction and planning; these are done on other NASA computers.

I mentioned the 10 year history primarily to indicate that there has been an opportunity here for the programming and NASA staff to get to know each other. Many of the people who worked on the original Mercury project are currently working on the Apollo project. They have developed an understanding of each other as individuals and this rapport between individuals is in our opinion a very significant factor in arriving at agreed upon specifications from which one can proceed to build a programming system. I should point out that we have other projects which lack this rapport, either because of personnel turnover in the military or because people don't like each other, and it is quite evident you cannot have an effective software engineering activity when you lack rapport.

It is obvious that Apollo was a real time system. What may not be obvious is that the system consists of a very large number of inputs and outputs, each of which is potentially unique and each of which changes for the next mission. There are many, many telemetry messages brought in from the space vehicle and from the tracking stations. The system supports a very large slide projection capability which can superimpose static displays over dynamic displays, all of these under program control. There is a very large number of displays which are monitored by the NASA monitors, usually assisted by one of the programming staff. These output displays are tailored to the individual who is going to sit there. Part of the design philosophy is to have the actual designer and engineer be responsible during the mission for supervising what he designs. That means that almost always your outputs are tailored to the personal interests of the man who is going to sit in front of the display console. It should be further pointed out that, at the time that programming started, it was not always possible to determine what the display unit would be in terms of hardware, so that in general the display systems required a symbolic generator. All display output programs were written in a standard form and a special program was written to convert the displays from the standard form to the proper device format.

The programming techniques were primarily assembly language and FORTRAN. However, it was necessary to build a special operating system called RTOS which initially was intended to look like OS/360 with real time appendages for device control. Because of schedule difficulties there was a divergence early in the development of RTOS so that, in fact, it is different from OS and is incompatible with it, but it has many of the same functions. 
Five of the six model 75's are close to one another. They all have large capacity storage and are interconnected through storage and through input/output devices. NASA's safety requirement is that a single mission be supported by a portion of one machine, leaving room for peak load, but backed up by other machines. If the primary machine that is running the mission fails, the job is switched to a secondary machine. The reason for the six machines is to support the mission, which may last several weeks, to support program development and to support simulations of future missions.

During mission simulation and during real missions, these machines are connected to a very large display and control room as well as to the input/output devices. The result of this is that during checkout of the real time programs, it is essential to dedicate some portion of this complex. That leads to each individual programmer using, on the average, 10 or 11 hours of computer time on a model 75 every month. This interested us because it is quite a high amount, but it turns out that it is not much higher than that for our other real time systems. We run an average of seven to eight hours a month on data-based systems. The systems run in batch mode for debugging. In some of our locations we use remote entry, at the moment in Houston we still use couriers. The programmers get one or two shots a day, but they know what to expect and in most cases they find that they can adjust their work to fit this type of schedule.

Let me turn to the question of the use of modelling techniques to help program development. Modelling has been used extensively in the Apollo support system project. A group of programmers was convinced that you should have a model of the real time system and they went about producing it. They came up with some things that were sufficiently convincing for NASA to permit them to build a model to run alongside the system itself.

They started on a 7094. They are now working on a S/360. Their model consists of a simulation of the hardware, the real time software, and the application programs. The model can be operated at various levels of detail; it can treat the mission programs as black boxes, simply looking at the inputs and outputs, or the program itself can be substituted to see how it actually works. Because this model is developed in parallel and runs constantly it is useful for such things as determining when you are going to run out of memory capacity or CPU speed. It has been used as a guideline in changing the hardware configurations. In addition it has been used for evaluating programming proposals and trying to determine which one looks most promising and should go to the head of the priority list.

Now it is always difficult to validate such a system and people tend to lack confidence in it until it is validated. This involves measuring the modelled system. In order to measure the operation of an actual system, a program called the Statistics Gathering System was built, which runs in cohabitation with the real time operating systems. It records information has continued to depend upon the simulation and has supported a group of some 25 people over the years just for this purpose. I indicate the size of the group because it indicates that not everybody is going to be able to support the kind of simulation that has been successful at Houston.

Finally, a comment on the question of schedule control procedures. Control consisted of, among other things: defining small modules, assigning decision making responsibility for modules to individuals who would commit to solve the problem, reviewing progress weekly, maintaining a detailed specification and change document, measuring and/or predicting system performance, monitoring financial progress, and requiring advanced reservation of computer test time. At one time NASA required that we develop a PERT chart. They have since withdrawn that as a requirement. In its place, a development and test plan is maintained and reviewed weekly by the IBM and NASA counterparts. It was useful, however, in the design stage when it provided a tool to identify things that had to be done and to show their interrelationships and the activities they depended upon and those that depended on them.

\section{6}

Ross: Your statistics on amount of debugging time and number of programmers indicate that each debugging run takes an average of 15 minutes. Is this right?

Aron: This is an average. In fact some runs will last for many hours or a day or more, when one is involved in testing the overall system rather than debugging modules. Considerable attention is being paid to finding ways to reduce this figure. 
Lang: What are the proportions of machine language to FORTRAN programs in the system? How was it decided which language to use for a given program?

Schorr: I understand that basically the orbit calculations, etc. are in FORTRAN. The modifications to OS/360 to produce RTOS were in machine language and that was basically the division. At the time I saw the project, there were 40 to 50 programmers working on RTOS, and approximately 250 programmers working on the application modules, and these people were writing almost completely in FORTRAN. About forty per cent of the total code was in FORTRAN.

Brown: I would like to know how many supervisory personnel are involved, how many levels of supervision there are, how many of these levels consist of people who are part time supervisors and part time programmers and how many levels consist of people who are able to read programs and do read the programs for which they are responsible?

Aron: This particular organization is heavy in programming background. It is a well known situation for an engineer to be managing programmers and not understand their product. Houston has been fortunate in having been built up for the purpose of programming. The typical organization starts with a first-line manager who will have six to ten people working for him. In general the first-line manager does not write portions of the program but he is responsible for the quality of the program. He always is capable of reading it and understanding it, and he may pick certain difficult parts of it to do himself. Above the first-line manager is a second-line manager. He will divide his time primarily between giving direction to the managers below him and coordinating the requirements with the user and his own boss. He is probably competent to read the code but he spends practically no time reviewing actual programs. I would assume that he gets involved only when there is a problem in testing and it is necessary to revise the design in some way. There are then two levels above that. The head of the project is a fourth-line manager; he is primarily an administrator, although he has a programming background.

Thompson: Could you comment on the division of responsibility between NASA and IBM, and on the problems associated with acceptance by NASA of the finished product?

Aron: The vendor in this case had total responsibility for the ground support programming package, meaning that NASA did not tell us how to do the programming and they did not look over our shoulders. On the other hand, as I explained before, it was impossible for us to make a step forward without understanding the user requirements, so there had to be close co-ordination 47 between the user and the IBM personnel. In general every group had a contact in NASA.

We did not do the mathematical analysis for the most part. NASA itself defined the problem, determined how the problem could be solved and gave it to us to program and verify. This is quite common in our business; we have to have people who are very broad in their ability to understand applications, but they are not often asked to generate the initial solution to a scientific problem.

On acceptance: any mission required from one to two months of actual real time simulation. The simulation during the latter period involved the actual NASA people who had to run the system. To ensure that the inputs were realistic they were recorded from actual instrumentation and fed in on tape or, at certain stages, they would be transported to the remote sites and fed in from the tracking station to simulate the actual communication links. At the end of this period of simulation, if you were ready to run the mission, you had acceptance. In addition, a formal acceptance of system components took place when test reports were approved.

Haller: What kind of specifications did you get from NASA?

Aron: That's hard to say. It is primarily a specification written out in English, with formulae, describing a procedure. It is not a programming specification in any sense. The problem has changed over the years; therefore they continually give us new inputs. The original problem was to put a single vehicle into an earth orbit. A succeeding problem was to put more than one vehicle into orbit and to find a way to transfer from one orbit to another in order to perform a rendezvous in lunar orbit, plus the control of a lunar landing. You will perhaps have noticed from television that the ground support center was always in communication. Part of the specification requires that this system be foolproof and as a consequence there are a tremendous number of safety features built in so that, if something unexpected happens, there are people sitting on the ground who will make decisions so as to 
correct the situation. The ability to support decision making on the ground is built into the program or at least into the program documentation.

Schorr: The human aspect of that is, I think, very important. There is no attempt to do it all by program reliability alone. For example, during one of the missions they found an error involving a module not releasing core store when it should. It just kept on claiming more and more core, and this was not noticed in the simulations. As a result, when the system was running, after a few days it got more and more sluggish. It was working, but nonessential functions were being dropped, because of lack of core space. Over a period of eight to ten hours a second system was brought up, by hand transfer of inputs to the system, very laboriously, and then a cut-over was made to this system.

Bugs are taken out up to the last day. Very often expedient decisions are made right before a mission to avoid using particular modules.

Falkoff: How do you construct the modules in such a manner that you can at the last moment leave some out and still have the system operate?

Aron: The easiest part of the system is the algorithm for solving the Newtonian equations. Where you really run into difficulty is in the human interface and in trying to understand what should be put out on the 48 displays and what should be put out on the radio links. This leads to a very early recognition that you must modularize things in such a way that if you had a telemetry experiment that you couldn't test you'd have to delete it. You couldn't allow it to interfere with the more formal, mathematical parts. A second consideration was that the schedule is much more important than the experiments, so that the management priority was to sacrifice experiments in the mission in order to satisfy a particular schedule for launching a vehicle. The modularity simply follows good rules of interface definition. The real time operating system was completely independent of the "problem modules". That meant that these modules could be extracted from the total system without too much trouble. Each named problem module could be worked on independently of other modules and it could be installed or extracted from the library master program, which could then be reloaded in the system. In addition, through the displays, you have the power to select which modules should actually be used.

Randell: It would seem to me that one of the main reasons for the undoubted success of the Apollo programming support system is its environment. It is part of a much larger effort, the total Apollo project, involving many other technological problems that had to be solved, and which could only be tackled a few at a time. Hence the support system has been done over many times; each time there has been a considerable change but it hasn't been a revolution. What worry me are systems which don't benefit from such a constraining environment, where software is the only technology that would be involved in a huge leap forward.

\subsubsection{The electronic switching system}

The Bell Telephone Laboratories system, subject of a paper by Ulrich reproduced in full in section 7.15, caused some discussion. The overall reliability of the system to date was summarized as follows.

Ulrich: We had an initial objective of downtime for the system of no more than two hours every 40 years, remembering that downtime is only caused by situations in which both processors are down or where there is such massive memory mutilation that we can't handle calls. Actually, we didn't adequately anticipate the second when we made our downtime objective. We thought primarily of how often we could reasonably afford to risk having both processors fail simultaneously, and we felt that perhaps once in 40 years was a reasonable objective. I might add that we've had in the order of 50 years of experience, accumulative over all our central offices, and we have not had an instance of this particular kind of trouble yet; so that prediction may not have been too far out of line. In terms of our overall achievement, however, instead of having two hours downtime in 40 years, in this 50 years of experience we've had a total of about 60 hours of downtime. Again, that's not bad; 60 hours in 50 years is about one hour per year, which is a fairly high degree of uptime for any computer system. This is the overall statistic since our first installation. More recently of course we've been quite a bit better. Our more recent statistics indicate 15 hours downtime per 40 years for large offices and perhaps five hours per 40 years in smaller 
offices. One of our big problems was to make sure that we would not get into a situation where we had massive memory mutilation, otherwise known as a crash, which would prevent us from setting up telephone calls, without our 49 recognize the situation. So we had to build facilities to make it possible to have a reliable scheme for recognizing that on an overall basis the program was cycling properly. The particular algorithm we used was to check the time required to go through all tasks, and if this time exceeded a certain specified amount, we became worried and we started performing progressively more serious initializations. I might add that our final step of initialization is to tear down all telephone connections, clear the memory, and start from scratch. This was not too favourably received by the Chairman of the Board of our corporation. When he first heard about this fact (after it had happened, of course) he is reputed to have remarked, "What idiot could have designed a system which deliberately tears down telephone calls?" He was not an experienced software man so he didn't appreciate the subtle beauties of initialization as a means of getting out of trouble.

The main types of programming error that caused crashes were the following:

1 We use pointers quite extensively and we interpret them literally without checking for range, primarily because of their extensive use and because of our need to conserve time on our main program legs. If a pointer is falsely overwritten, you quickly get into a situation where you start propagating trash throughout the memory and this can get you into trouble.

2 Another situation which has occurred in a number of cases is the unchaining of a lengthy list of pointers. This unchaining might occur because of almost simultaneous events which, when tested in the laboratory, did not show up. But when the random situation in the field turned up, the almost simultaneous event in effect violated what turned out to be a guard interval and caused problems.

3 One thing which happened a number of times and was a little embarrassing is that we falsely switched in a store which turned out to be faulty and which was perhaps being tested with a test pattern. You can imagine what happens when you interpret a chequer board pattern as the data concerning all the telephone calls in the system!

Hoare: I derived much encouragement from Ulrich's description of two of the most common kinds of programming bug, because it throws some credit on the people who organized a NATO Summer School in Grenoble a few years ago. I gave a paper on techniques for ensuring by some form of absolute, rather than run time, check that pointers are being used correctly. Edsger Dijkstra gave a paper on techniques for ensuring that unusual sequencings of events didn't cause unpleasant results. If there is a conflict at this conference between the theorists and the practical men, I hope this example will help to resolve it.

The problems of maintaining compatibility also came up.

Ulrich: Dijkstra's remark about puritans falling in love with the source of their misery also applies, I think, to telephone central office designers. One of the important sources of our misery is that we have to live and communicate with a very extensive existing telephone plant that is quite old and that was never designed to work with a modern system. This is a classic example of having to be downward compatible for an indefinite period. For example, the telephone instrument is really an improved version of an instrument designed for the Strowger system, which was the first automatic telephone central office, invented in 1889, and which is still highly competitive in the small office range.

50

Schorr: Ulrich's point about the necessity for the central office to be designed to be downward compatible with all the existing telephone company equipment is all too relevant to software engineering in general. We are getting into an era in software production where we will have to be very careful about how we design our interfaces so that we are downward compatible. Going from second generation to third generation it was simple to do some emulation. Maybe now the operating system interfaces and so on will be another piece of baggage we'll have to carry along as we go further and further.

\subsubsection{The "Super-programmer project"}

One further case history was given, this being of an experiment in which a single extremely expert programmer attempted to produce a system which was, though small by some peoples' standards, of considerable size. The 
report was given by Aron during discussion of the scheme proposed by Seegmüller (see section 2) for the design and implementation of a system.

Aron: I would like to describe an experiment we performed in which a single programmer, Dr. Harlan Mills, undertook to reproduce a project that had been started in a typical "army of ants" fashion and was still under way. He hoped to do in six man months what was regarded as being essentially a 30 man year project. His approach was essentially similar to Seegmuller's scheme; his language was PL/1 and he used it from the top down. He started out using PL/1 as a pidgin English; he simply wrote out his abstract ideas in an English form which was closer to PL/1 than it was to conversational English and, as he proceeded into more detail, he generated the remaining parts of the PL/1 statement. The essentials to his procedure were (1) develop the programming system structure and (2) develop the data files and the intercommunication system within the program. He found that, having done that, it became fairly straightforward to fill in the algorithms within each program module. Now, we tried to study this project in order to determine whether it was a success or failure but we ran into difficulties; namely, that he didn't finish it in a single effort. There were interruptions, other projects along the way, so that it wasn't clear exactly what the results were. But it was clear that it didn't take six man months to do the job; it took more like six man years, which still wasn't bad since it was substantially better than the other group. On the other hand, during the period of the experiment, the other group's objectives changed substantially because the customer changed what he wanted. The program was called the "definitive orbit determination system" and its purpose was to build a history of orbit programs and orbit observations for space agency and air force satellites. It was to be an interactive system whereby scientists could come up to a computer, request the assembly of information from old satellite activities and study it to determine such things as the geodetic shape of the earth, distances from the earth to the moon, etc. The system itself was, I would guess, around 50,000 instructions and the file was rather large. The structure of the information in the files was diverse because it was picked up from miscellaneous jobs, none of which had been performed with the intent of storing the results in this file. Dr. Mills had the role of chief programmer and his function was to do everything regarding generation of the program down to the final code and testing of the code. In order to assist him, however, he trained his secretary 51 to be his interface with the computer. Naturally, he found that he had to develop a small programming support system in order to enable her to communicate with the computer, the way a secretary would, rather than the way a programmer would. And he had a little help to get that programming system built. The second thing he found was that in order to write a program in PL/1 he had to spread out, like, 36 manuals on his desk. In order to get around this, first of all he requested us to assign him (part time) an expert PL/1 programmer, a programmer who was expert on the internal details of OS/360 and a few other consultants. Finally he acquired what we call a programmer-technician, a trainee programmer without a degree, whose job was primarily clerical support.He found that, in order to simplify the languages that he used (PL/1, JCL and linkage editor), he had to rewrite the manuals for JCL and linkage editor. This turned out to be fairly worthwhile because it was then easy to turn the description of these procedures mechanically into a set of control cards that his secretary could conveniently use.

The estimate of the man-power required increased from his original estimate. A second point that was far more significant was that, because the job he was doing was running parallel to an existing job, we chose not to give him a direct interface with the customer. We didn't want to confuse the customer about how many different IBM groups were supporting him, so we took one of the systems analysts from the contract project and made him the simulated interface with Dr. Mills. This turned out to be a mistake, in my opinion, because it shielded Dr. Mills from all of the actual problems of customer relations.

We have a second project of this type now, dealing with an information bank for a New York City newspaper and here we do have direct contact with the customer. Progress is substantially slower, and it is obvious that Seegmuller's first point is an extremely difficult one to reach since we are dealing, at least in the case of application systems, with users who don't really know what they want; moreover, they don't realize what they want until they're into the project itself.

Now let me turn to the question of why we don't use this technique in our many other projects. First of all a chief programmer such as Dr. Mills is a very unusual individual We started out referring to him as a superprogrammer; the term may not be accurate but it is descriptive because among the other 2,000 programmers there are not more than a few of equivalent capability. The capabilities required are extremely good program- 
ming knowledge, extremely good application knowledge and a desire to work very hard on this type of problem. It was necessary in the orbit determination system to understand sufficient mathematics to develop the orbit equations and so forth, as well as understand programming. In addition it was necessary to understand a subject that is dominant in many of our application systems, namely, the problems of interface with displays and with analog input such as radars and data acquisition devices. Now, in our remaining projects, because we cannot depend upon the existence of a super-programmer in any given project and, because we must transfer our people around in order to apply the right number of resources at the right time to a contract, we have found that it is important to build our systems without dependence upon any particularly strong individual. So we set up a management and control organization that makes the best out of the resources that are normally available. If we find that, within a project, we do have a great strength then we find that the individual concerned naturally introduces more advanced ideas. But we find that it is impractical to plan our project with the confidence that we'll find such an individual in 52 the beginning. Our current goal is to fit the best implementation approach to each new project.

\subsection{The sources of problems in large systems}

One of the subjects that was discussed at length was the extent to which there are technical as opposed to managerial problems in the design of large systems. (Debate on managerial problems per se, has not in general been reported on; though working material which, in the editors' opinion, significantly supplements the material given in the Garmisch Report has been included in section 7, i.e. the paper by Aron.)

Aron: We made a study of about a dozen projects, though not in a very formal manner. However, our results were convincing enough to us to set up a course on programming systems management. The nature of the study was "Why do our projects succeed or fail?" We took as "successful" a project that met its requirements on schedule within the budgeted dollars and satisfied the customer. On this basis, out of 10 or 12 projects that we examined, we had one success and a whole lot of failures. We analyzed the reasons for failure, as given to us by the project managers, and by people who had performed previous evaluations of the projects. They gave various reasons behind the failure of the projects, virtually all of which were essentially management failures. We ran into problems because we didn't know how to manage what we had, not because we lacked the techniques themselves.

Buxton: There are serious management problems of course-but are all the problems managerial in nature? I get the strong impression, from Aron for instance, that his view is that it is completely and totally a management problem and that his establishment is entirely satisfied with classical assembly language and FORTRAN programming techniques, which were well developed many years ago. Have you no need ever for, say, recursive programming techniques, for example?

McGowan: The problem is managerial in the sense that it is a managerial responsibility to learn new techniques and develop new tools to solve our problems. However, the Exec 8 system generation routine is essentially an example of a technical, rather than a managerial, solution. We have problems with multiprocessor interlocking and scheduling, and with storage allocation, for example.

Scalzi: One of the reasons that OS/360 is such a big management problem is that there are technical problems to be solved. We should not need to use anywhere near the same number of people again if we re-do OS/360. One of the major problems is testing the system; particularly since different components are produced in widely separated areas. Many of our problems arise from our inadequate means of specifying software.

Aron: I was not trying to claim that all problems are managerial in nature; rather that management decisions are required to take advantage of any technology that is available.

Perlis: Take, for example, OS/360 and its Job Control Language. I have yet to hear a good word about JCL. What kind of managerial structure is it that 53 doesn't filter a new proposal against existing knowhow so that, for example, JCL would have the right properties of a language? It is failures like that which are technical failures on the part of managers, and which are largely responsible for major difficulties in the system. I think that, by and large, the managers wouldn't know a good technique if it hit them in the face.

\section{NATO SOFTWARE ENGINEERING CONFERENCE 1969}


Hoare: Basically all problems are technical. If you know what you want to do and you have the necessary technical background, there is no point in making a great management problem out of it. Obviously a certain amount of resource control and personnel work have to go on but that's all. On the other hand you might have a task which is difficult in that you don't know what you have to do, and that even if you did you wouldn't know how to do it. Since this is a very difficult task you can start out with the hypothesis that it will take a great number of people. Following down this slippery slope of reasoning, as soon as you have a very large number of people you have a very large management problem. But it all originated out of the fact that you didn't know what you were doing, or how to do it. Perhaps because we don't have large teams in Europe we tend either not to understand or perhaps to understand too well what the Parkinsonian slope is in this case; and we feel more inclined to cry that the Emperor has no clothes.

A subject that was discussed at much less length than its importance would seem to merit was the real meaning and significance of the concept "module"

Falkoff: On what basis, according to what discipline, have these people who built large systems decided what is a module?

Buxton: 4096 bytes!

Aron: For example: to take a very empirical look at the question Falkoff asked, a programming module can be defined in a very large system by looking at what a designer hands over to a programmer to implement. In general, we found that he hands over a programming module which ends up as about 400 to 1,000 machine language instructions when it is finally built. That was the smallest unit of the system. The size depended upon the degree of detail that was considered important by the designer, and it varied from one man to another. It also varied according to what he thought the capability of the implementing programmer would be.

Ulrich: For some of our programs this was a relatively straightforward process. A module was the response to a particular telephone event. For example, one of our modules is the response to the recognition that a customer has picked up his phone and wants to get a receiver into which he can dial. This was a reasonable algorithm for a portion of our program. However, it did not help us at all so far as setting up the large body of common programs.

\subsection{Support software for large systems}

During the conference a brief presentation was given by H. M. Brown of a system being used inside IBM to assist in the development of large-scale programming systems. The slides that he used during the presentation are reproduced as Figures 1 to 11.

Brown: CLEAR, standing for "Controlled Library Environment and Resources" is a programming development support system designed to help 54 IBM development programmers manufacture large systems. It aims to assist the administration and the accomplishment of the program development cycle. The second slide (Figure 2) gives more detail as to the administrative support provided.

The third slide (Figure 3) indicates some of the facilities provided for programmers. The preprocessor interface, for example, allows the programmer to use simple verbs, rather than complicated and lengthy JCL programs, for such jobs as modifying a piece of source code, followed by reassembling it, and possibly testing it. The What Used/Where Used mechanism will scan a module and tell you the macros, LPSW instructions, supervisor calls, etc. that it uses. The so called "Delta Concept" allows you to avoid changing the actual source text but instead make your changes in a "Delta" data set, which is an increment to the source text. Therefore anything which was previously released is (hopefully) unchanged and will still be there with the next release of the system.

This so far has just been the CLEAR system, a batch processing system, which is several years old by now. The CASTER system (Computer Assisted System for Total Effort Reduction) is much newer, and is an exten- 
SLIDE 1

Major Objectives-CLEAR System

I. Administration of Program Development Cycle

11. Assistance in Accomplishing Program Development Cycle

* Specification

* Flowchart

* Development

* Document

* Integrate

* Release

* Maintain

Figure 1

SLIDE 2

I. Administration

* Programming Parts Warehouse

* Flexible, Efficient File Maintenance

* Common Inter-Center Environment

* Disaster Recovery Capability

* Maintenance of Statistics

* Authorization Scheme

* Minimum Hardware Requirements

* Time Accounting

* Management Report Network

Figure 2

\section{SLIDE 3}

II. Development

* Preprocessor Interface

* Central Library Facility

* Powerful—Efficient File Maintenance

* Positive Level Control

* What Used/Where Used Mechanism

* Delta Concept

* Unique Sequencing Scheme

* Powerful-Flexible Data Extraction

* Statistical Data Maintenance

* Authorization Scheme

* Support for any OS/360 Program

* Convenient Data Recovery Facility

* All Features of OS/360 Available 
sion of CLEAR: the two together being known as CLEAR-CASTER. This system provides on-line access to the information which the CLEAR system maintains using both typewriter and simple graphic display terminals. It also provides a communications centre for passing messages between people.

The "Node Structure" referred to on slide 6 (Figure 6) is the means by which a filing system can be defined in a hierarchical structure, probably closely akin to the development group's organizational structure. Modules are attached at nodes. Conversational processing covers such things as syntax checking. You can also use conversational mode in connection with standards checking and flowcharting.

SLIDE 4

$\mathrm{CC}$

Computer Assisted

System for Total

Effort Reduction

Figure 4
SLIDE 5

CASTER

An Extension to Clear

A Development Tool

An Integral File System

An On-Line Keyboard-Graphic Terminal System

A Communication Center

Figure 5

The whole of the data base for your programming system is contained in structural form in the filing system, as an interlinked set of nodes. The $\mathbf{5 6}$ possible classes of node are given in slide 7 (Figure 7). For example: a node can contain a source code module, documentation to be used by technical writers, standards to be adhered to in writing code, specifications, etc. The second column indicates the types of data set available: source code, delta, object code, listing of an assembly or compilation, information data set, etc. (The information data set contains statistics concerning, for example, changes made to modules.)

\title{
SLIDE 6
}

\section{CASTER Functions}

\author{
Node Structure \\ Terminal Data Entry and Manipulation \\ Conversational Processing \\ Remote Job Entry and Reply
}

Figure 6

\begin{abstract}
The next slide (Figure 8) indicates the facilities provided for programmers by the system. When a programmer creates a module he copies into the beginning of it a set of module specifications which existed as a skeleton data set in his library. Keywords have to be answered according to prompting information. Eventually this will be joined by the source code and flowcharting information that he produces. Facilities for syntax analysis of individual program statements are provided. A keyword data set can be given at a major node of a library to hold a set of standards for the source code in that library. For example: labels might have to start with a certain sequence of letters, program jumps relative to the current values of the instruction counter should be of limited size, etc. A command could be used to check that the source code adhered to these standards. Conversational debugging, which should be available in six months, is designed to help in the early development of a module rather than during later stages of system development, since object code is executed interpretively. The phrase "Library for Integration" relates to facilities for integrating source code modules developed in various different programming centres around the world into a single system.
\end{abstract}




\section{SLIDE 7}

File System

\begin{tabular}{l|ll} 
Node & Class & Type \\
\hline \multirow{1}{*}{ Module } & SRC \\
Macro & DEL \\
Plan & OBJ \\
Preface & LST \\
Specification & IDS \\
Message & KWD \\
Documentation & TXT \\
Control Info & Module Class- \\
Form & Module Specs \\
Standards & PL/1 \\
& & Source Code
\end{tabular}

Library . Comp. Module . Class . Type . Gen

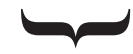

Node Name

Figure 7

\section{SLIDE 8}

Support for Development/Implementation

Programmers:

Correlation Among Module Specifications, Flowchart, and Source Code

Syntax Analysis

Standards and Convention Checking

Conversational Debugging

Library for Integration

Figure 8

On slide 9 (Figure 9), a Defined (keyword) Dataset is in effect a questionnaire which is copied to a node and then filled in, e.g. module specifications. Answers could be machine checked. Designers can update module specifications and send messages alerting people to the changes. Similar facilities, concerning control information, are directly available to management personnel.

\section{SLIDE 9}

Support for Designer/Planner/Architect

Defined Datasets Attached to Major Nodes For All Types of Information

Maintain Continuity of Design Information Throughout Development

Figure 9 
SLIDE 10

Support for Management

Easy Access of All Control Information

Reporting

Automatic Message Transmission

Figure 10

SLIDE 11

Writers

Entry of Data

Entry of Control for Formatting Processor

Immediate Access of All Source Material

Text 360 Processor

Figure 11

58

Finally slide 11 (Figure 11) indicates that technical writers can also have access to information concerning, for example, module specifications and flowchart statements associated with pieces of source code, and can use a text formatting processor (currently TEXT/360) to help format documentation, written at the terminal.

Oestreicher: It would appear that the CLEAR-CASTER system could be very valuable to users, and could reduce the machine time they use in developing large programs; is it available to them?

Brown: It is an internal tool, and to my knowledge there is no current intention to release it. A system like CLEARCASTER requires extra machine time, which has to be balanced against the benefits that might accrue in the long run to a complete development project. We haven't proved yet, one way or the other, whether it will significantly reduce the time taken to complete a project.

Scalzi: OS/360 is developed in a decentralized manner, using groups in 15 to 20 different places all around the world. Our major problems have been not knowing all the interactions which exist between components, and not 59 having a structure which would enable us to define an interface formally and then police its use. (For example, we have found that tables that we laid out in 1966 are no longer adequate and we want to change them but are not completely sure in all cases who uses them.) We are trying to get tools that will help with these problems.

We use the CLEAR system. We have a central library in Poughkeepsie where everything is collected. Everyone sends programs into the central library for each release. When we come up with a mechanism for interface control and monitoring we will do it in this one central location. One of our practical problems with CLEAR is turnaround time. We are therefore very interested in CLEAR-CASTER, which is essentially "on-line CLEAR".

Aron: We find that our projects often develop a CLEAR-like library control system which keeps track of what is going on. We have an experimental timesharing system which is used for building system programs. The concept here is a little bit different to the CLEAR-CASTER concept in that our system is intended for use from the beginning of a project to the end, and it is intended to be an interactive program development tool in addition to being a library control tool. Its major weakness at the present time is that you can't extract yourself from it in order to go into a batch operation. 
Kanner: (from "Software engineering tools") "If the premise is accepted that computer manufacturers' establishments have the most to gain from the introduction of software engineering tools, it is pertinent to ask how this introduction might be accomplished. Some enlightenment might be gained by observing the initial experiences of a project manager trying to develop such tools, this work being done in a small research department of a not so small computer manufacturer.

The project, which started out with the title System Programming Tools, was a direct response to pressures from immediate management that might be paraphrased: 'Make some tools for those guys out there,' and quickly discovered that those guys out there really did not want any tools other than possibly those of their own construction. In fact, the existence of about five small, possibly bootleg, implementation language projects was discovered within two software production divisions of the company. The project manager came to the conclusion that:

1 He should positively not produce any type of small tool that was already in production elsewhere, and should probably not devote much effort anyway to individual tools.

2 He should direct his efforts to producing an integrated collection of tools operating as a single system in the sense of, though not necessarily as ambitious as, CLEAR.

3 In any event, he should devote considerable time to studying the problems of software production with the purpose of identifying the areas for which possible tools might provide the greatest assistance.

About this time, the name of the project was changed to Software Engineering Tools, and the bandwagon had a passenger!

The obvious theme of these remarks is that there has as yet been regrettably little experience with the employment of tools in software production. There are gross uncertainties as to their cost, effectiveness and reliability. In parallel with research on tools and techniques as such, a much more mundane sort of research should be prosecuted: the systematic experimental application of software engineering tools to real or contrived software projects for the purpose of evaluation and improvement thereof." 


\section{SOFTWARE ENGINEERING EDUCATION}

A session at the conference was devoted to discussion of this topic. In an opening speech Perlis proposed three questions:

$1 \quad$ Is there a real distinction between software engineering and computer science?

2 Given that the answer is yes, is there a need for education in software engineering as a separate discipline?

$3 \quad$ What form should university courses in software engineering take?

The discussion that followed ranged widely over these and other topics, including the question whether computer science is well enough established to be taught to undergraduates and whether it has any discernible basic principles.

The majority of the discussion is reproduced, subject to the customary amount of editing, in the following section. (A quotation from Strachey given in section 4.1.6 is also relevant to this subject).

Perlis: The first question to which we might address ourselves is: Is there a difference between software engineering and computer science? I suspect there is a big difference between them and so do some other people. Doug Ross, for example, has devoted a great deal of effort in his own bailiwick and has been driven out of the temple (by the money changers) still firmly believing there is something called software engineering. What he practices, be it software engineering or not, is certainly quite different from computer science.

If there is a subject called software engineering, I think we ought to ask ourselves if we need an output from our educational system different from that which we are now producing. I would argue that, in the United States at least, we are not producing software engineers; indeed our general organized Ph.D. programs are going to make it difficult for us to produce software engineers and the situation is likely to become worse as time goes on. So, if there is a distinction between computer science and software engineering and a need for people trained in the latter, then half the universities had better start doing something about it.

In computer science, at least in the United States, we do have a large number of quite good Ph.D. programsgood in the sense that they are rapidly accelerating towards ossification, which establishes in a reasonable way their legitimacy. I think that all of us who are in universities at least have a pretty good understanding of the nature of a computer science doctorate program: a healthy dose of logic, automata and computing theory, a diminishing dose of numerical analysis, one or two courses in advanced programming of one sort or another, some artificial intelligence, and some of this and some of that. Then one does thesis topics in any of the areas that are covered by Computing Reviews.

Evidently computer science is able to generate its own problems, understood and appreciated by only its own people. There doesn't seem to be any shortage of Ph.D. thesis topics in the Ph.D. area; there doesn't seem to be any shortage of positions for people educated in such programs in 61 other Ph.D. programs in computer science, so everything is really quite healthy in the academic sense.

But is there something else: a different point of view, a different emphasis that we find in computer science programs both in industry and in the universities?

Bauer: We have had a committee in Germany working on a curriculum. We have called the subject "Informatik". The course will include both theoretical work and workshops in programming. We expect our students will have the choice of becoming either computer scientists or software engineers.

Feldman: I occasionally do a certain amount of work in industry and find out some surprising things in this way. For example: practical people do not read the literature, even though sometimes there are real solutions to their problems to be found there. The people who have been through an educational program in computer science seem more effective in this sort of respect. 
We are trying to face up to this problem at Stanford by setting up a degree course in applied computer science, aimed at producing effective people for industry. Now suppose we give this a first degree, can people really choose between theory and practice as a career? I think not; I think it is very hard for people with a natural tendency in one direction to move in the other.

Perlis: I agree with Feldman that one does not become a theoretical person by studying mathematics or a practical one by ignoring it. That's not really an issue. The real issue is: what are the important problems and how much effort do we sink into them? The way Ph.D. programs are organized in computer science it is inconceivable for a professor and for his students to sink, for example, five to seven years on a long term project. In almost no department of computer science was it required that every student take a course in operations research and management science and yet I claim that to be a fundamental course in software engineering; as fundamental as automata theory and more fundamental than any mathematics course I can think of.

Galler: I don't think it's unheard of for a professor to work with his students for several years on a project. Some of our students have been around for seven years working on system projects; some of them get so involved they never finish.

I would like to describe a course which we've given twice now, and which I think is a software engineering course. We have taken either two or three professors as a team with 20-25 students and given them a project. The first was a mathematical graphics system, the second was a BASIC system. We've given them the complete job of designing, stating the specifications, dividing up into groups of two or three each, implementing, describing their work to each of the other groups, designing interfaces, documenting and making the thing work. In each case the product was something that was almost useable; in one semester you can't necessarily complete something of that scale. This kind of experience goes beyond writing a small class project type compiler and gives a kind of software engineering experience. I think it can be done, and I think maybe one should begin to recommend that for a curriculum.

Ross: This is the same as teaching electrical engineering by building a 63 crystal set, then a one-tube receiver and then something else. That was indeed the way it started but not the way it is now. It isn't the proper way to set about teaching an engineering subject.

Falkoff: I think that software engineering is too restrictive a name for the curriculum; it must include some hardware work. Also, I always thought that engineering had to do with economics questions; it seems quite essential that a discipline which has to do with problems of complex use of resources should contain some operations research and related courses.

Needham: I have a cautionary tale which supports in many ways what Perlis said at the beginning. A few years ago our graduate students took part in a large project; they did things that fitted in with other people and they worked all hours and they wrote a lot of programs in an imaginative way, but with very strict discipline on the interfaces. Academically it was not a success. It didn't lead to prompt or very excellent theses, but on the other hand the people produced were perhaps the best contribution to the software industry we could have made.

Aron: The field of software engineering is mainly concerned with people writing programs for money. The expense on education is an overhead which is normally minimized to a standard level; programmers understand how to apply one high level language or maybe a macro-assembler to a real problem. The deeper concepts may never reach them. .

McClure: Universities have a considerable problem with which I sympathize and yet I condemn them. I have watched this at close range. Let me see if I might illuminate the problem to see where some of the solutions might possibly lie. I think it's at the heart of whether there is a dichotomy between software engineering and computer science. I believe that the computer science departments are turning out a reasonably high grade of individual, you might call them computer scientists, most of whom go into stocking the faculties of other computer science departments. This has been true of a number of other disciplines as well-the medical schools have had the situation. In fact some medical schools specialize in turning out faculties for other medical schools. I think that part of the problem lies in the fact that there are really two classes of universities, at least in the United States. We might describe them as those that are in and those that are out. Having been associated with one that would have to consider itself out I can speak with some authority. It seems that the ones that are in are determined to maintain their in-status. Those that are out desperately want to get in, which they feel they can best do by

\section{NATO SOFTWARE ENGINEERING CONFERENCE 1969}


emulating those that are in rather than charting for themselves an independent course of action which would suit their local purpose.

Most of the ones which are spread around the United States are community and industry supported, and industry urgently needs their output. The intent of these graduates is to go into and man the works of all these places as they do in electrical engineering and other disciplines. Now in fact, however, external support of universities hinges to a large extent upon their relative reputation. The industries don't make independent judgements for themselves as to the quality of the product but look at the universities' relative ranking among other academic institutions. These, of course, are judged by other academics.

Let me say why I think this leads into a difficulty. Graduates with bachelors degrees in engineering traditionally are equipped with a bag of 64 tricks that they're willing to perform for their employer. Their employer knows that, if a man has a bachelors degree in engineering, he is capable of doing certain things. A masters degree means that he is capable of doing more tricks, and a doctorate in engineering means that he is capable of yet additional tricks. Now on the other hand, Ph.D's are supposed to be capable of extending the state of the art, as contrasted with doctors of engineering who are merely capable of performing high class tricks. At the present time most people feel that they cannot succeed academically without getting Ph.D's. In fact graduates themselves only want Ph.D's because doctors of engineering are looked down upon-when in fact industry most desperately requires doctors of engineering because it has shops that it must man. It wants people not capable of extending the frontiers in science in some abstract theoretical sense but people who are knowledgeable about what really goes on.

We have said that teaching OS/360 is in a sense bad, because OS/360 is a bad system. On the other hand, medical schools reliably teach pathology — disease structures. The only way one can learn to recognize mistakes is by studying things that are wrong. That means that you must study listings and study documents much as a medical student spends many hours poring through a microscope. This is very painful and unfortunately it's not glamorous but it's at the heart of what I call software engineering. Until universities get around to believing that in fact there is a requirement for people who really understand how things happen we'll not make any progress in an engineering discipline. We may make a great deal of progress in computer science, but we'll not make any progress in software engineering.

Teitelman: I agree with McClure's remarks on the relative status of the Ph.D. and doctor of engineering. Speaking from personal experience, when I had to choose what program to follow in school, one of the factors that influenced me was the knowledge that the Ph.D. meant something in terms of status and freedom that might perhaps outweigh the merits of an engineering program.

Ercoli: Those who have to be trained to use computers in accountancy don't need to study automata theory or some theoretical substitute for it. We need to train these people: it is far more urgent than training more scientists or engineers to use computers.

Oestreicher: I think that one thing which is happening in universities is that they are becoming more down to earth. I have often heard of compilers and even operating systems coming out of universities which, if they hadn't been done by so few people, might have been thought to be large software projects.

The only contribution we can make in industry is to expose young people to the problems they meet in industry, without discouraging them too much. They need to be allowed to make their own mistakes in order to learn; they need to be exposed to customers and so on. Quite often they respond by doing things you thought to be impossible!

Dijkstra: A Dutch definition of a university professor is someone who casts false pearls before real swine!

Otherwise my position differs greatly from that which Perlis describes. I am engaged in teaching, at graduate level, in producing one variety of "mathematical engineers". The most powerful test that I know of for an 65 applicant to be one of my students is that they have an absolute mastery of their native tongue: you just need to listen to them.

I find it difficult to find worthwhile research subjects and think it wrong to teach material which I know will be obsolete in a few years. What finally remains isn't so much. You have to teach a grasp of method, a sense of 
quality and style. Sometimes you are successful. This cannot be all of computer science or software engineering. It may well be that it is a rather small subject but it may take very good people to work in it.

A neglected part of software engineering is the area of hardware design. If a software engineer cannot specify hardware design, who can?

Strachey: Computing science has been under some attack on the grounds that it isn't software engineering. I propose to attack it on different grounds. I think we should seriously ask ourselves the question: is computing science?

Recently I did a small survey as to whether computing is suitable as an undergraduate subject in an English university. I did this by grading all the topics I could think of under the headings of relevance and state of development. The premise is that it is clearly wrong to teach undergraduates the state of the art; one should teach them things which will still be valid in 20 years time: the fundamental concepts and underlying principles. Anything else is dishonest.

The gradings for relevance ran from "clearly relevant and essential" to "part of another subject" (like numerical analysis) and those for state of development from "well developed with theorems, laws and text books" to "a fruitful field for research". Note, incidentally, the importance of text books. They are designed to be taught from; they are quite different from treatises and even further from research papers. Now, it turned out that all those subjects which score highly for relevance score very low on state of development and vice versa.

Until we have a sufficient body of topics which are important, relevant and well developed we cannot call the subject a "science". I am quite convinced that in fact computing will become a very important science. But at the moment we are in a very primitive state of development; we don't know the basic principles yet and we must learn them first. If universities spend their time teaching the state of the art, they will not discover these principles and that, surely, is what academics should be doing.

I do not for a moment underestimate the importance of the state of the art in engineering. Clearly it is essential and furthermore from engineering practice we must get our experience and material from which we develop theory. But, before teaching students we must get our basic principles right.

Woodger: I wonder what a basic principle in computer science would look like if I saw one. Seriously, in what terms might it be expressed? It's no good using unexplained terms like "language" or "compiler"; we have very few clear terms in which to express any fundamental principle of computer science. Would any professor here tell me a single basic principle?

Perlis: Some principles: every interesting program has at least one variable, one branch and one loop. An interesting program is one whose number of execution steps is not linearly proportional to the length of the text.

Strachey: And at least one bug!

Perlis: No, I don't accept that that is a principle.

66.

Woodger: Well, the concept of "variable" is far from trivial, at the least.

Falkoff: I'm not a university professor but I would like to say something about basic principles. Last summer we assembled a group of school mathematics teachers and set out collecting material to give them a basis for working with APL in teaching high school mathematics. We found there were only a few essential notions that the pupils needed in order to use a programmable machine. These were:

1 The notion of assignment, which I think covers the idea of a variable.

2 The notion of a conditional expression or branch.

3 The notion of function definition.

I propose that these three ideas are central to computing and will remain so.

Gotlieb: One basic principle with regard to programs is that execution time and store utilization are interchangeable. In a good program, you can improve one only at the expense of the other. 
The problem of lack of text books was discussed by Lampson.

Lampson: In my experience of trying to teach this subject in university, one of the most annoying aspects is that there is nothing to teach it from. You sit down to teach a course, any course, and you have to start from scratch. You have to think for yourself about what to do, and the amount of time it takes to do a quarter-way decent job is more time than you can afford to spend on preparing one course. It seems to me that the situation could be improved. There are several areas in which very substantial contributions could be made by a small group of people, working for two or three months, trying to produce a document which would describe, in some sense, some essence of the state of the art. I suggest three areas in which this could profitably be done

1 What programming is all about.

2 The basic technology for writing compilers (not just for describing programming languages).

3 The basic principles for writing operating systems.

The right group of three or four people, working for two or three months, could produce a document of perhaps 150 to 200 pages that would really serve as a standard for the field, in that it would provide a base from which one could teach, and in many cases a base from which one could talk and think. This would eliminate a great deal of our terminological difficulties and a great deal of the re-thinking that one has to do when one embarks on a new project.

The attempt should be to tell what is known, not to break new ground and not to produce a complete or finished work in any sense; that would be disastrous. Neither do we need to produce anything like Knuth's books, for which I have the greatest admiration, but which can only be viewed as an encyclopaedia. I feel very confident that this could be done.

Schorr: I could provide facilities for such an enterprise. 


\section{WORKING PAPERS}

\subsection{List of working papers}

Aron, J. D. Estimating resources for large programming systems

Aron, J. D. General remarks on Apollo programming support

Bauer, F. L. and Goos, G. Requirements for programming languages for basic software

Bauer, $R$. Towards computer-aided production of large application programs

Bemer, R. W. Straightening out programming languages

Bemer, $R$. W. A note on software specifications

Bemer, $R W$. A note on implementation correctness

Bemer, $R$. W. A plea for consideration of computing as it will be, not was

Brown, W. S. Software portability

Burkinshaw, $P$. How to avoid some pitfalls in software project management

Buxton, J. N. A note on sharing software

Clingen, $C . T$. Program naming problems in a shared tree-structured hierarchy

David, E. E. Adoption of new tools and methods in creating large systems

Dijkstra E. W. Structured programming

Donner, H. Systems for multiple or changing environments; properties required for software portability

Ebbinghaus, $K$. Organization and human factors

Falkoff, A. D. Criteria for a system design language

Feldman, J. A. Towards automatic programming

Galler, B. A. Construction of generators to produce programs for specific applications

Galler, B. A. and Perlis, A. J. Criteria for the design of a language

Gotlieb, C. C. and MacEwen, G. H. System evaluation tools

Hoare, C. A. R. An axiomatisation of the data definition features of PASCAL

Hoare, $C$. A. R. The problems of software development

Holmann, F. Computer-aided software production for process control

Hopkins, M. E. Computer aided software design

Italiani, $M$. On progress control of a software project

Kanner, $H$. Software engineering tools

Kanner, $H$. Tools

Kjeldaas, $P$. M. Facilitating efficiency and generality by using run time information in compilation

Lampson, $B$. W. On reliable and extendable operating systems

Lang, C. A. Languages for writing system programs

Lemma, A. and Rossi, E. The production cost of the software for little programming systems

Lowry, E. S. Proposed language extensions to aid coding and analysis of large programs

MacGowan, Jr., J. M. UNIVAC 1108 instrumentation

Needham, $R M$. Software engineering techniques and operating system design and production

Needham, R. M. and Aron, J. D. Software engineering and computer science

Oestreicher, M. D. Operating systems

Poole, P. C. and Waite, W. M. Machine independent software

\section{7}

Randell, B. Interacting sequential processes

Reenskaug, T. Some notes on portable application software

Reynolds, J. C. A set-theoretic approach to the concept of type

Ross, D. T. Reproduction of slides for bootstrapping of the AED system

Schorr, H. Compiler writing techniques and problems

Schwartz, J. I. Report on literature search on management 
Schwartz, J. I. Analysing large scale system development Seegmüller, G. Definition of systems

Sharp, I. P. Systems design for a changing environment

Teitelman, $W$. Toward a programming laboratory

Thompson, $K L$. Software quality control at Michigan State University

Tixier, $V . O / S$ writing systems

Ulrich, $W$. Design of high reliability continuous operation systems

van der Poel, W. L. A simple macro compiler for educational purposes in LISP

Warshall, $S$. Software portability and representational form (abstract)

Wiehle, H. R. Means of communication in operating system 1/TR440

Wirth, $N$. The programming language PASCAL and its design criteria

Woodger, M. and Duncan, F. G. An overdue comment on ALGOL 60

\title{
7.2 Estimating resources for large programming systems
}

by

J. D. Aron

\begin{abstract}
This paper presents a Quantitative Method of estimating manpower resources for large programming jobs which represents a simple, feasible guideline. The method combines program structural data with historical data on the productivity of programming groups. This data is then adjusted to cover non-programming resource requirements. This is not a precise method and, in fact, it is not as good as an estimate based on sound experience. But it is offered as an aid to estimators working in unfamiliar areas and as a verification of estimates obtained by other methods.
\end{abstract}

\section{Introduction}

It is very common for the cost and schedule of large programming systems to exceed initial estimates. This is most often due to changes in the functional specifications but, even where the specification is fixed, it is difficult to estimate software resource requirements accurately. Not only does the main resource-manpower-vary widely in productivity and quality but the secondary resources, such as machine time and publications support, are frequently unavailable at the appropriate times. Most important, every large system is an aggregate of so many elements-program modules, organizational interactions, logistics, etc.- that no manager can precisely determine the amount of work to be done. On the other hand, managers of some large jobs, such as the Mercury-Gemini-Apollo mission support systems, have learned how to meet rigid schedules successfully. By examining the methods used in successful areas, it is possible to draft guidelines for use on new projects.

\section{9}

This approach has been taken by IBM's Federal Systems Division (FSD). It draws on FSD experience in many large jobs since 1959 as well as on other relevant background. The result is a set of guidelines that, while subject to error, are useful for arriving at a quantitative estimate of manpower resources. The method is taught in the FSD Programming Project Management Course, where it has been recommended as an aid to estimating projected work (during proposal or early design stages) and as a cross check on estimates of work in progress. Among the more than four hundred managers who have taken the course were many experienced managers who corroborated the quantitative guidelines and few who contradicted them.

\section{Large systems defined}

For the purpose of this paper, a large programming system is a "structured aggregate of elements that satisfies a set of functional and performance specifications" and that requires

- $\quad$ more than 25 programmers

- $\quad$ more than 30,000 deliverable instructions

- more than six months' development time

- more than one level of management. 


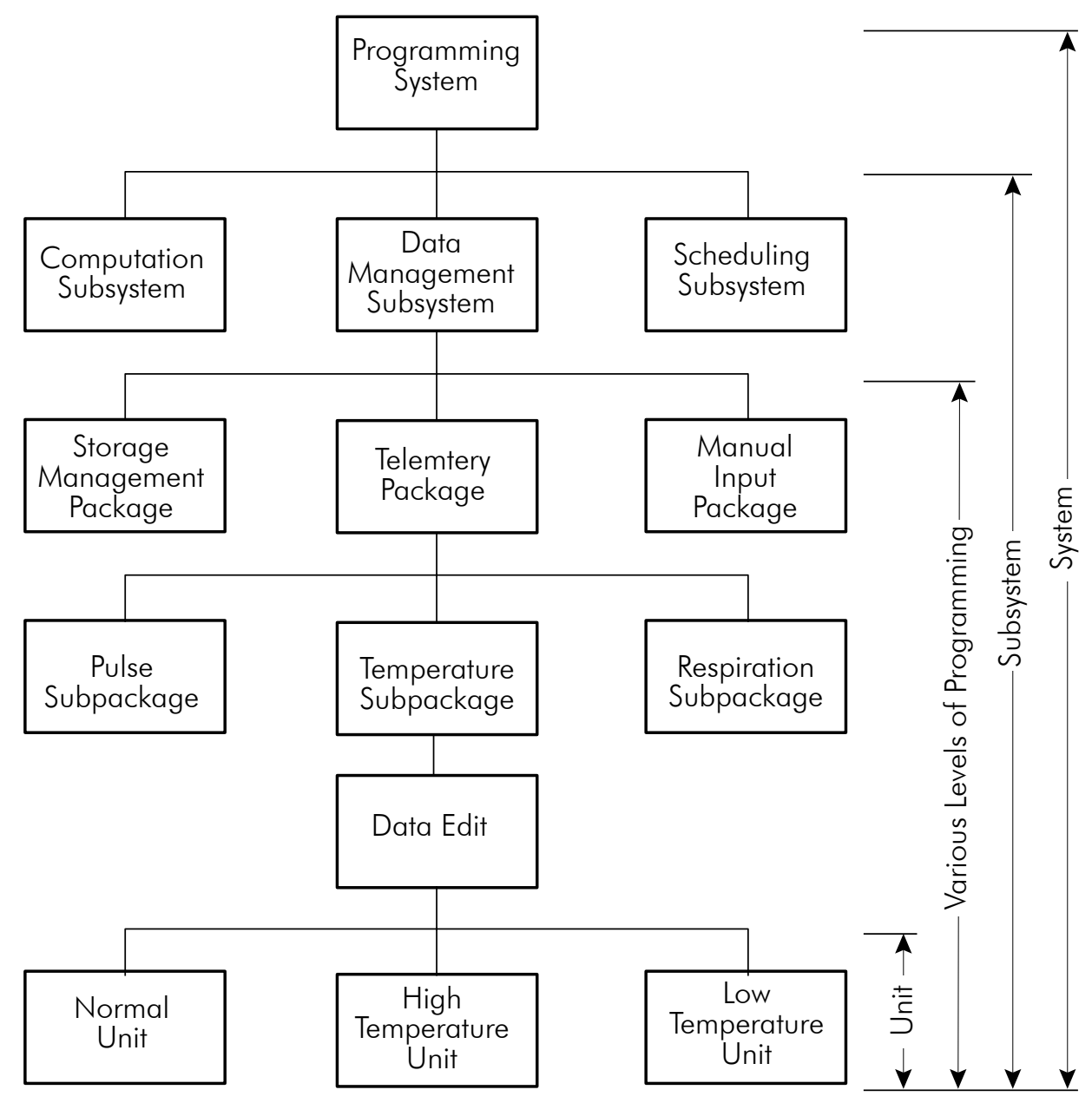

Figure 1. Organization of system components

\section{0}

These arbitrary parameters describe a system which has enough elements interacting within it to create a management problem. Such systems are large enough to absorb the imprecision of a quantitative estimating system. For example, if there are 25 programmers it is possible to ignore their individual differences and use an average productivity parameter in a quantitative formula. The very size that creates large system problems is turned to advantage.

A system contains subsystems which are further divided into smaller hierarchical structures until the smallest programming element is reached (Figure 1). The smallest element is referred to as a "unit" in later sections.

System design consists of defining the components and their interactions; the definition is a "specification." Detailed design consists of defining the subsequent lower levels of the structures until each unit is described in terms of its function and performance. Detailed design of a single unit is done by the implementing programmer; therefore, the unit can be viewed as the smallest element of interest to the system architect. This last point is significant because, although different designers work to different levels of detail, each designer is rather consistent in deciding when to stop designing and hand the work over to the programmer: 


\section{System development life cycle}

The system is built according to a life cycle, as shown in Figure 2.* The life cycle shows the activities that must take place in system development and the level of manpower required to do the job. The chart is truncated at both ends, omitting concept formulation at the beginning and maintenance and improvement at the end. The slope of the boundaries in the upper half of the chart represents the fact that activities overlap; some programming may 71 start before all packages have been designed or some test and integration may begin before the last unit is debugged.

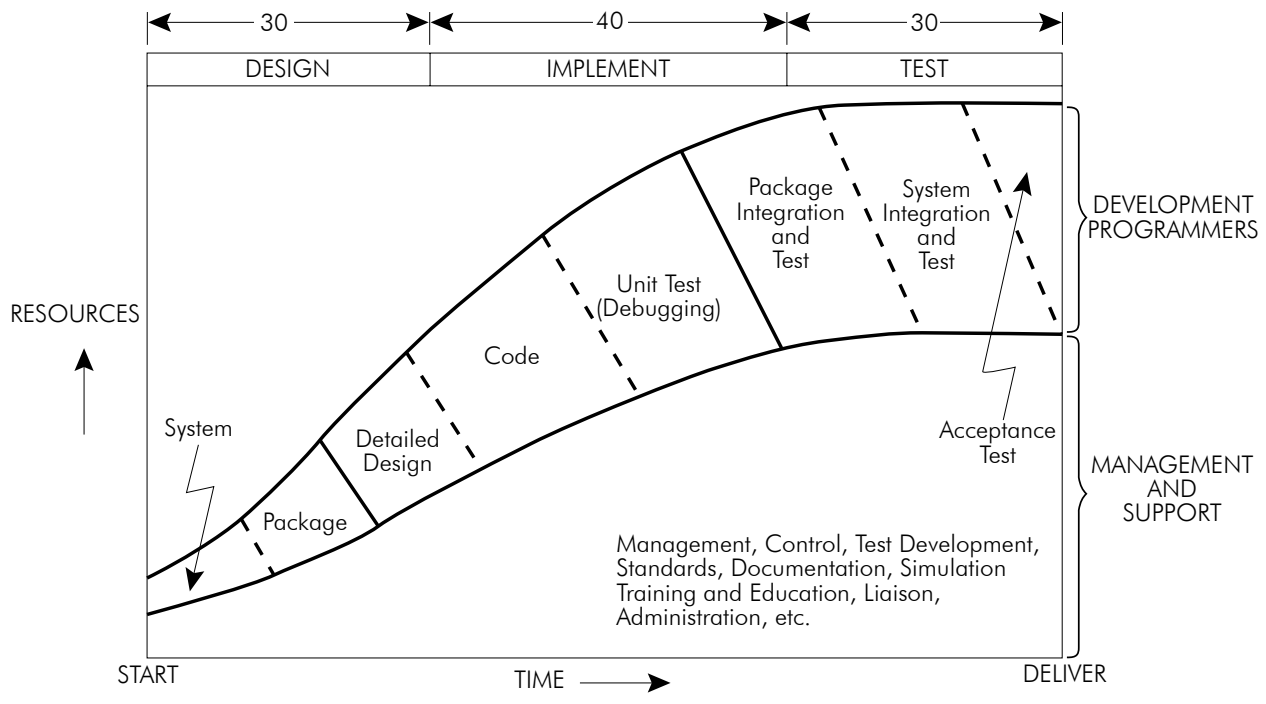

Figure 2. System Life Cycle

Certain gross features of the life cycle are important when using a quantitative estimating guideline because the result of the estimate applies only to certain parts of the chart. The total estimate is an extrapolation based on the following simple ratios:

1 The ideal project is planned so that about 30 per cent of the elapsed time is used for design, 40 per cent for implementation and 30 per cent for test. (Maintenance, which is omitted from consideration here, continues throughout the life of the program.)

2 The management and support resources are 40 to 50 per cent of the total resources.

3 System design resources are negligible compared to total resources. (This point is made simply because the quantitative method does not consider system design resource requirements explicitly since they can be ignored for practical purposes.)

4 The duration of the project is determined by the manager to meet a delivery commitment or to allow orderly recruiting. (The formulae produce estimates of man-months. It is up to the manager to spread them according to a schedule.)

5 The life cycle chart includes all the manpower resources needed to do a specific job. It includes management and clerical support assigned full time to the job but does not include headquarters management or other manpower that may have an occasional responsibility in the project. Neither does it include overhead activities, such as machine operations, publications, etc., which are used as a service.

\footnotetext{
*This chart and much of the background material is adapted from Mr. A. M. Pietrasanta's work at IBM on programming system development. The System Development Corporation's (SDC) Programming Management Project, under Mr. V. LaBolle and others, and the FSD Programming Project Management Course and Guide, prepared by Mr. P. W. Metzger, were also referenced.
} 


\section{Methods of estimating}

Before describing a quantitative guideline, it is necessary to re-emphasize that a "guideline" is just that. It cannot be more than an aid to decision making. As Mr. Pietrasanta said in his paper "Resource Analysis of Computer Program System Development,"

"The problem of resource estimating of computer program system development is fundamentally qualitative rather than quantitative. We don't understand what has to be estimated well enough to make accurate estimates. Quantitative analyses of resources will augment our qualitative understanding of program system development, but such analyses will never substitute for this understanding."

This is why, when methods of estimating are ranked, the list is headed by the Experience Method Experience on a large programming system can be carried forward to similar systems. An ability to anticipate resource requirements and a cautionary attitude toward contingencies are part of one's experience. As long as a manager does not try to apply his experience to a larger or substantially different job, he can do no better than to base his estimates on experience. The remaining methods are substantially less reliable, including the Quantitative Method described later. In order of desirability, the methods are: Experience, Quantitative, Constraint, Units of Work.

\section{Experience method}

This approach takes advantage of experience on a similar job. In order to use the method, the new job must be clearly specified, at least down to the package level. This permits the estimator to compare the new system to one or more completed systems. At this point, the estimator can assume that like tasks take like resources. He can obtain the base data from his own 72 experience or from that of other people as long as he knows he is comparing genuinely similar projects. If the two projects are alike in size and content, minor differences in algorithms or utility routines can be allowed for by adding a contingency factor to the total estimate: the contingency factor should be less than 25 per cent. As in any method, it is wise to lay out the design in detail to permit the men who must implement the job to make their own estimates on their portion of the job. Their estimates will also be based on experience and should be more precise than the total estimate. In other words, two similar orbital mission systems of 150,000 instructions each may be within 25 per cent of one another in effort. Two telemetry data processing programs of 1000 instructions each may appear in these systems. A specialist in telemetry data processing should be able to estimate his tasks to within 10 per cent.

The major problem in the method is that it does not work on systems larger than the base used for comparison. System complexity grows as the square of the number of system elements; therefore, experience with a small system cannot account for all the things that will have to be done in a large system. Neither will the Experience Method apply to systems of totally different content. The Quantitative Method is applicable in such cases.

\section{Quantitative method}

The Quantitative Method to be described in this paper is based on programmer productivity in terms of the number of deliverable instructions produced per unit of time by an average programmer. The deliverable instructions include the source statements and data descriptions written by the programmer in a macro-assembly language. The method is not precise; it is necessary to adjust the answer by large amounts (see Factors affecting the estimate). The estimator should never allow himself to treat the answers as anything other than approximate representations of system size or manpower requirements.

\section{Constraint Method}

This method is equivalent to taking an educated guess. Based on schedule, dollar, or manpower constraints, the manager simply agrees to do the job within the constraints. His decision is unrelated to the design complexity of the system. The merit of this approach is that it is often possible and, in some cases, beneficial for the user and the developer to reach mutual agreement on the constraints. Once agreed, they can proceed to define a set of specifications which can be achieved within the estimate. In cases in which either party does not understand the consequences of constraint estimating on product specifications there is great risk of overrun.

Referring again to Mr. Pietrasanta, he points out that a given set of specifications will require a certain number of man-months to produce. These man-months can be spread over a short or a long period of time. If the man-months are not available, though, the specifications will have to be reduced in scope or the job will not get done. The Experience

\section{NATO SOFTWARE ENGINEERING CONFERENCE 1969}


Method and the Quantitative Method assume that specifications are fixed and that the man-months are estimated to satisfy the specification. The Constraint Method, if properly used, holds the man-months fixed and varies the specification to fit.

\section{Units of work method}

The history of small programming efforts, particularly in administrative and business data processing, shows far better estimating performance than large systems. This seems to be due to the use of a special case of the $\mathbf{7 3}$ Constraint Method, which is called "Units of Work" for lack of a more descriptive name. In this method each programming task is defined in such a way that it takes one programmer 4 to 8 weeks. Each task is designed, implemented and tested independently of all other tasks. This approach eliminates the interactions that cause trouble in large systems but programs written this way cannot be linked to form a large system. The purpose of mentioning the Units of Work Method here is to highlight its limitations and to suggest a better method to estimators with large systems to build.

\section{Factors affecting the estimate}

An estimate prepared by any method is based partly on fact and partly on assumption. It also contains errors due to imprecise inputs. Managers are advised to make sure that every estimate they prepare or review contains enough information. As a minimum they must know how the estimate was prepared and what the input data was. In addition they need the assumptions and the contingencies used by the estimator. With this data the managers can evaluate the estimate and make knowledgeable judgments. If they decide to reduce the estimate, they know how far they can go in trading cost for risk. They also know where they can reduce risk by changing an assumption, for instance: substituting a more experienced programmer for a weak one. The result of their evaluation will be a commitment to perform the job for a given price. (Whereas an estimate may contain a contingency factor, a commitment does not.) Since managers depend for their success on the successful performance of their people, good managers invariably use the assumptions and contingency data to improve the project. The employee must have enough confidence in his manager to believe this, or else he will hide a little "fat" in the estimate and not admit it.

The variables in estimating are so large they cannot be ignored. The SDC Programming Management Project spent several years analyzing a large amount of historical data to identify the factors that affect programming cost the most. Starting with 104 variables in the programming process, they obtained statistical data showing 11 to be significant enough to use in estimating indices. Although the Quantitative Method does not use the indices, it does recognize that the variables can affect the estimate and it permits major adjustment of results. The key variables fall into three groups:

1 Uniqueness

2 Development environment

3 Job type and difficulty.

Uniqueness is concerned with the familiarity of the development team with the hardware, software and subject matter of the project. If they are unfamiliar with any of these because they lack experience or because the item is new in the field, the cost of the project will increase by an unknown amount. Development environment involves customer/ programmer capability, working relationships and organization. Development environment also involves geographic dispersion and working facility quality. Weak people, poor communications channels due to organization or dispersion, unpleasant facilities, etc., increase cost by reducing productivity. These two groups of variables must be evaluated by the manager and reduced to a single factor, which he uses to modify the estimate. The adjustment in the Quantitative Method can range from about -25 per cent if all the factors are favourable to +100 per cent if all are unfavourable. The adjustment is chosen subjectively, based on a judgment of how much the factors differ from the norm for the estimator's organization.

\section{4}

Job type and difficulty deal with the number of system interactions due to program and data base elements and the relative difficulty of various types of programs. These variables are accounted for in the quantitative formula. 


\section{Quantitative estimating procedure}

The quantitative estimating procedure starts with a design. The degree of detail in the design depends on how much time is available. At proposal time the system design may be completed only through the package level. After the job has entered the design phase, re-estimates can be based on lower levels of detail. At any stage, some structural detail is required for the size of the system to be determined. At least one package in the system should be laid out to the unit level. The package selected for this purpose should be the one considered the most difficult by the estimator. The detailed design of this computation accomplishes two things: it ensures that there are no mathematical or logical problems in the package that cannot be handled and it gives a measure of system size. It is important that, since a large system involves many man-years of effort, it is worth the trouble of a good design at the start.

After the first step of system design, the process proceeds to:

- Estimate number of deliverable instructions

- $\quad$ Estimate difficulty of programs and duration of project

- Determine man-months for programming

- $\quad$ Adjust for higher-level language

- Extrapolate man-months for the project

- Adjust result

- $\quad$ Schedule effort.

This procedure is repeated at a frequency of the order of once a month to obtain increasingly better estimates as the project develops.

\section{Estimating number of instructions}

The key to estimating system size is found in the design. Since the system is an aggregate of elements, its size can be determined by counting the number of elements and multiplying the result by the average element size.

Deliverable instructions $=$ number of units $\times$ average unit size

The number of units is estimated from the design by carrying at least one package design down to the unit level. This shows how many units are required for that particular package. The number of units in other packages or programs can be estimated by:

- $\quad$ Experience with similar packages

- $\quad$ Sketching the design for the package

- Using the same number obtained for the base package. The number of units in the system is simply the sum of the units in the packages.

The average unit size depends on the operating habits of the designers. Typically, units written in assembly language contain 400 to 1000 instructions. The average length is becoming smaller as modular programming is emphasized. Units written in high level language are about one-fourth the size but generate deliverable programs of comparable size. Although this is a wide range, any individual manager, designer, or architect tends to normalize his design so that the resulting units fall within a narrow range. By examining his habits he can determine the average unit size that he assigns to his people. The average is perfectly adequate for the purposes of the quantitative estimate.

\section{5}

\section{Estimate difficulty}

Some programs take more time to program than others. Three levels of difficulty: easy, medium and difficult, are used to improve the estimate. The three levels result from many factors in the job, but they are conveniently

characterized by the number of interactions found in various program classes.

1 Easy-very few interactions with other system elements. The class includes most problem programs or "application" programs. Any program the main function of which is to solve a mathematical or logical 
problem is probably in this class. Easy programs generally interact only with I/O programs, data management programs and monitor programs.

2 Medium-some interactions with other system elements. In this category are most utilities, language compilers, schedulers, I/O packages and data management packages. These programs interact with hardware functions, with problem programs, with monitors and with others in this class. They are further complicated by being generalized enough to handle multiple situations: e.g., I/O from many different I/O devices or management of data files with variable numbers of indices.

3 Difficult-many interactions with other system elements. All monitors and operating systems fall in this class because they interact with everything. Special-purpose programs such as a conversational message processor may be in this class if they modify the master operating system.

As a project proceeds, the implementers learn their job better. The "learning curve" is the rate of improvement. The productivity data in Figure 3 shows that the rate of improvement for easy and medium programs is good, but difficult programs are still hard to produce after many months of experience.*

In the Quantitative Method the degree of difficulty determines the productivity values to be used. Figure 3 shows the levels of difficulty (rows), numbered 1,2, and 3. One way to estimate difficulty is to pick a value from 1 to 3 representative of the overall character of the system. If the result is a decimal fraction, interpolate between rows of the table., The second way to estimate difficulty is to break the instruction count into three parts, estimating the number of easy units, medium units, and difficult units separately. Then calculate the man-months for each batch and total the results.

\begin{tabular}{|c|c|c|c|c|c|}
\hline & Difficulty & $\begin{array}{c}\text { 6-12 } \\
\text { Months }\end{array}$ & $\begin{array}{c}12-24 \\
\text { Months }\end{array}$ & $\begin{array}{l}\text { More Than } \\
24 \text { Months }\end{array}$ & \multirow{5}{*}{$\begin{array}{l}\text { Very Few } \\
\text { Interactions } \\
\text { Some } \\
\text { Interactions } \\
\text { Many } \\
\text { Interactions }\end{array}$} \\
\hline Row 1 & Easy & 20 & 500 & 10,000 & \\
\hline Row 2 & Medium & 10 & 250 & 5,000 & \\
\hline \multirow[t]{2}{*}{ Row 3} & Difficult & 5 & 125 & 1,500 & \\
\hline & & $\begin{array}{c}\text { Instructions } \\
\text { per } \\
\text { Man-Day }\end{array}$ & $\begin{array}{l}\text { Instructions } \\
\text { per } \\
\text { Man-Month }\end{array}$ & $\begin{array}{c}\text { Instructions } \\
\text { per } \\
\text { Man-Year }\end{array}$ & \\
\hline
\end{tabular}

Figure 3. Productivity table

\footnotetext{
* The data in this table summarizes historical data from IBM projects involving system programs-1410, 7040, 7030, System/360 OS/PCP-and application systems - Mercury, SABRE, banking and brokerage, FAA. It has not been formally validated, but it has been informally checked against other IBM and some non-IBM estimates. It is interesting to note that the contents of the table seem as good in 1969 as they were in 1960. It is not clear why this is so. There are several speculations to explain the apparent lack of any increase in productivity in the industry:

I The capacity of the computer to give improved debugging service limits the programmers' output.

2 Untrained programmers enter the field at such a rate that the average ability to use programming knowhow is equivalent to about one year's experience.

3 Programmers fail to learn better methods.

And, the thought that I favour:

4 Programs are getting more difficult at the same rate that programmer skills improve. This implies that as computers become more powerful we use them to tackle harder problems.
} 


\section{Determine man-months}

Using Figure 3, it is a simple matter to select a productivity rate and divide $\mathbf{7 6}$ it into the system size to obtain the resource requirement for writing programs.

$$
\text { Man-months }=\frac{\text { Deliverable instructions }}{\text { Instructions per man-month }}
$$

The selection of a row in the table was described as a function of job difficulty and job type. The selection of a column depends on the proposed length of the project. There are two reasons for multiple columns:

1 The numbers are more convenient to use because they are more appropriately scaled to system size.

2 The columns are adjusted to include learning.

\section{Example}

At this point, a number has been generated representing a portion of the system resource requirement. Suppose the system of interest has seven major packages. One of these is concerned with data management, which contains three main programming activities. One of these is telemetry, which is unique to the development group so it is selected for detailed design. In developing the detailed design a set of subpackages is laid out, each of which is broken into its basic units. There are a total of 50 units. Lacking other data, the number of units in the system could be taken as $7 X 3 X 50=1050$. More likely the other packages are better known and specific unit counts can be generated for each. The total unit count this way is, say, 800. Suppose now that a poll is taken of the key men who will design and assign units to programmers. Each man reviews his previous results and explains how large his unit assignments turn out to be on the average.

The average for all responses is 500 assembly instructions and associated data descriptions. Further, assume that one package (150 units) is judged to 77 be difficult, two (200 units) to be medium, and four (450 units) easy. One year is planned for system development. The procedure will produce an estimate as follows:

$$
\begin{array}{ll}
\text { Deliverable instructions } & =800 \times 500=400,000 \\
\text { Difficult } & =150 \times 500=75,000 \\
\text { Medium } & =200 \times 500=100,000 \\
\text { Easy } & =450 \times 500=225,000
\end{array}
$$

Using column 2, an answer in man-months will be obtained.

$$
\text { Man-months }=\frac{225,000}{500}+\frac{100,000}{250}+\frac{75,000}{125}=1450 \text { man-months }=120 \text { man-years }
$$

\section{Adjust for higher-level language}

Up to this point, only assembly language source statements have been considered. Productivity using higher-level languages such as FORTRAN, COBOL and PL/1 is highly variable. An experimental factor of 2:1 improvement over assembly language is suggested. Thus if the estimator counted deliverable instructions generated from source statements he could divide the man-months obtained from the table by 2 to adjust for the advantages of the higher-level language. If he entered the table with the number of high-level source statements and data descriptions, he could multiply the resulting man-months by 2 . This allows for 4 deliverable instructions to be generated, on the average, by each high-level statement.

\section{Extrapolation}

Figure 4 illustrates the activities in the life cycle that are estimated from support are not included. They must be brought in by extrapolation, as follows: 


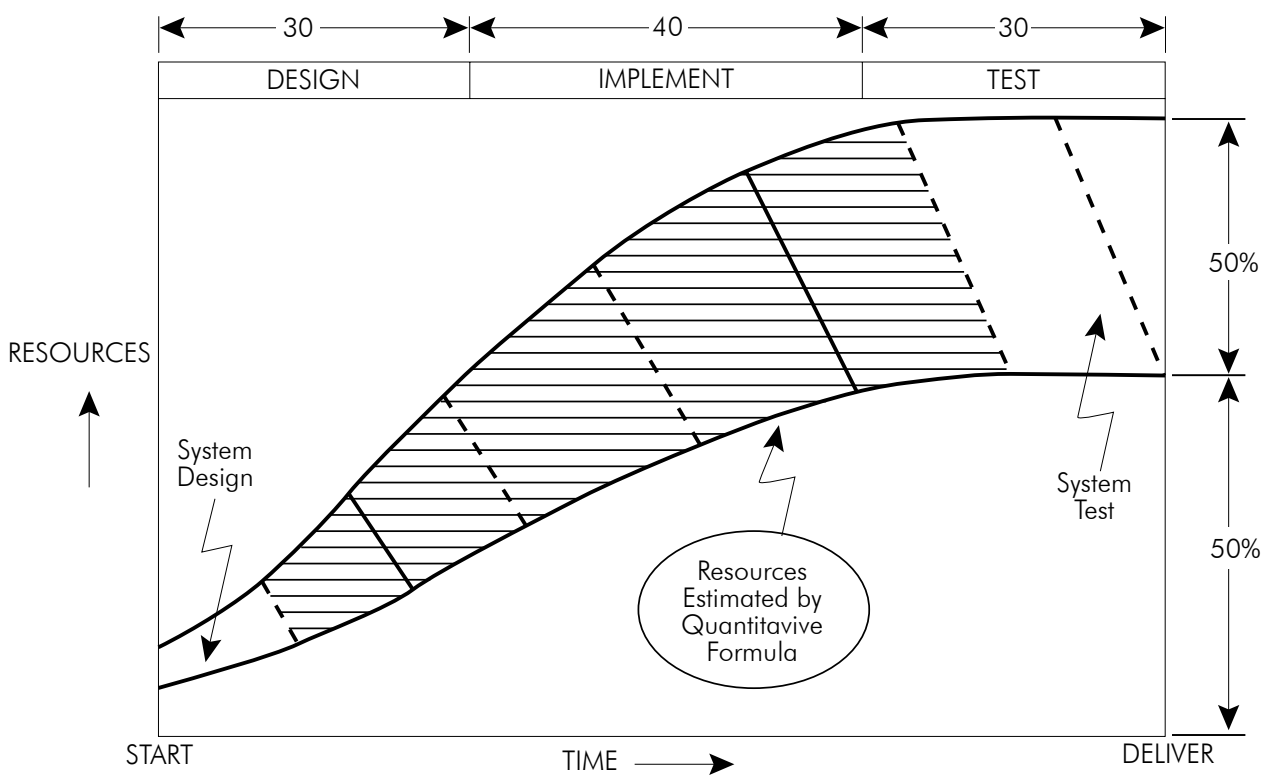

Figure 4. Scope of quantitative estimate

1 System design can be ignored-it is less than one per cent of an effort the size of the above example.

2 System test represents about half the test time or 15 per cent of the schedule. But since the number of people on hand at that time is very high system test actually uses closer to 20 per cent of the resources, not counting management and support.

3 Management and support utilize approximately half of the system resources.

4 The extrapolation is done by the following formula:

$$
\begin{aligned}
\text { System man-months } & =2\left(\frac{\text { man-months }}{0.8}\right) \\
& =2.5 \times \text { man-months }
\end{aligned}
$$

In the example of a 400,000 instruction system the total resource estimate would be 3625 man-months or over 300 man years.*

\section{Adjust results}

The productivity table assumes the use of average programmers organized in a normal pyramidal hierarchy. That means that there is a range in experience from many junior people to fewer senior people. The formulas also assume average uniqueness and development environment. No adjustment of the result is necessary unless the project deviates in an obvious way from the norm. Such an obvious deviation would be the assignment of all junior people with no experienced technical people to lead them. Another would be the requirement to use the first machine of a new line-one that has never been used before in an operational environment. The amount of adjustment appropriate is entirely subjective, since it represents the manager's confidence that he can overcome the effects of deviation.

\section{Scheduling}

The development of an actual performance schedule and budget will not be discussed in this paper except to point out that the estimate projects only resource needs. Plans, schedules, budgets, reviews, etc., are needed to get the job going in a controlled way. The form of the schedule and budget varies from organization to organization, so no format guidelines are uniformly acceptable. A couple of general points are useful:

1 Schedule documents should include activity networks (PERT charts) and bar charts.

* A The method can be used for much smaller systems, although it is not advisable for jobs under 10 men or under six months' duration. These cases are not big enough to cancel out the inherent errors of the quantitative method. 
(a) Activity networks are essential in design because they are the best tool for showing system interactions.

(b) Bar charts are good at all times because they show who is assigned to each task and when significant events (checkpoints and milestones) occur.

As shown in Figure 5, management attention is directed to interactions during design and test. It shifts to the components themselves during implementation. The activity network is most valuable, then, during design and test but it is a burden during implementation. If it is not properly maintained it should be dropped in implementation for economy and the bar chart should be retained.

2 The schedule of manpower follows the life cycle. Other activities that must be scheduled include machine time and other job shop services such $\mathbf{7 9}$ as publications. These activities tend to fall in the last onehalf to one-third of the life cycle.

(a) Machine time can be estimated in gross terms by knowing how many machine-hours each man in the organization uses each month. (A survey report by Mr. Ralph Arthur and this author showed that in FSD 7 to 8 hours debugging time per man per month was predictable from the history of several dozen projects.)

The gross estimate can be spread by allowing 2 to 3 hours during implementation building to about 20 hours in test, but averaging 8 hours per man per month over the last 50 to 70 per cent of the project.

(b) Other service activities are best estimated by the specialists in those areas. This means that their inputs must be requested during the estimate process.

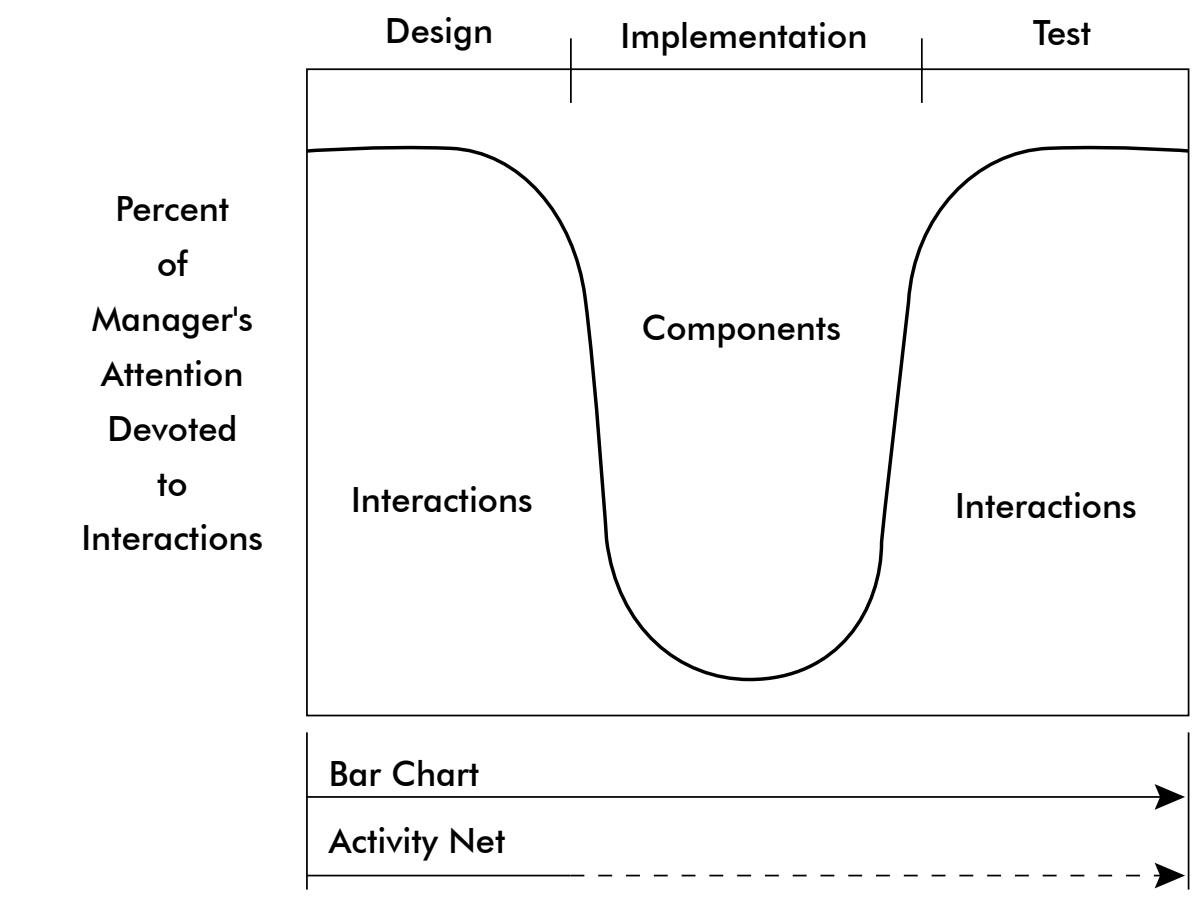

\section{Useful Tool}

- - - - Desirable Tool

Figure 5. Manager's attention curve

\section{Conclusion}

The Quantitative Method of estimating manpower resources for large programming jobs is presented as a simple, feasible guideline. It is not being presented as a precise method and, in fact, it is not as good as an estimate based on sound experience. But it is offered as an aid to estimators working in unfamiliar areas and as a verification of estimates obtained by other methods. 


\subsection{Software portability}

by

W. S. Brown

\section{Abstract}

A program or programming system is called portable if the effort required to move it into a new environment is much less than the effort that would be required to reprogram it for the new environment. This paper consists of a general discussion of software portability, followed by a description of the portability plan for ALTRAN-F, a special purpose programming language now being implemented.

\section{Introduction}

A program or programming system is called portable if the effort required to move it into a new environment is much less than the effort that would be required to reprogram it for the new environment.

Most software is not portable. The resulting situation is becoming an increasingly serious obstacle to progress in computer science. Many programmers spend much of their time doing work that has been done better elsewhere before. More often than not, programs described in journals are unavailable to the readers. Every project must be implemented from the ground up and, when a computer is replaced, many running programs are effectively lost.

Problem oriented languages were originally expected to provide a cure for this situation but they have succeeded only to a very limited extent. In Section 2 of this paper we examine the reasons for this. In Section 3 we classify software according to difficulty of achieving portability, and in Section 4 we describe the portability plan for ALTRAN-F (see Reference 1), a special purpose programming language now being implemented.

\section{Problem oriented languages}

A problem oriented language is a language which allows the programmer to describe a procedure in terms of the problem rather than the machine. It was originally hoped that programs written in a problem oriented language could be moved from one machine to another, simply by recompiling them, but experience has made us aware of several serious pitfalls:

1 Problem oriented languages come in many dialects. In fact each compiler defines a somewhat different source language from every other. A program that runs well on one machine is very likely to collapse on another. Furthermore, because of inadequate documentation, it is often difficult to learn what the differences are.

2 There maybe no existing language which is well suited to the task at hand. For example, one may need to handle numbers, character strings, lists, structures and input/output. Most languages tend to specialize in one or two of these. FORTRAN and ALGOL are excellent for numerical computing, while SNOBOL is especially designed for the manipulation of character strings. LISP specializes in lists and COBOL is excellent for structures and for input/output. PL/1 and ALGOL 68 do all of these things but both are ponderous; furthermore PL/1 is supported only by IBM and ALGOL 68 has not yet been implemented at all. Dialects often incorporate extensions to overcome some of the deficiencies, but a program using the extra facilities cannot easily be moved.

81

3 Only a few languages are widely available.

4 The use of a problem oriented language may entail an unacceptable loss of efficiency. If a compiler is to produce efficient object programs then the language must permit the programmer to express his intentions clearly. If he is forced into circumlocutions, the compiler may compile efficient code for what he said, but it can hardly be expected to compile efficient code for what he meant. A compiler can go to tremendous extremes of program analysis and searching for special cases in order to optimize its object code, but if so the compiler itself will be bulky and slow.

5 A problem oriented language cannot readily provide portability if any significant part of the problem at hand is machine dependent. 


\section{Classes of software}

We define four classes of software in increasing order of the difficulty of achieving portability.

\section{Small procedures}

A small procedure performs a conceptually simple computation on (or transformation of) arguments provided by its invoker. Input/output can usually be avoided, and there is a good chance of an adequate language being available.

\section{Large subsystems}

A large subsystem provides for the solution of a multitude of problems, each of which can be described without reference to the computer. Facilities for input/output must be provided and there may be no available language which permits natural expression of all parts of the subsystem.

\section{Compilers}

A compiler presents all of the difficulties of a large subsystem and also includes a module, the code generator, which is inherently machine dependent. In spite of the obvious difficulties there is hope and even some evidence that portable compilers can be produced. If so, both the necessity and the harmful consequences of having many dialects of each language may be eliminated.

\section{Operating systems}

An operating system must not only deal directly with the machine, as a compiler does, but it must also satisfy service requests which are explicitly stated in terms of the machine. The hope for portability rests on the observation that most service requests are not so stated and that large parts of typical operating systems involve ordinary processing of numbers, strings, lists, arrays and structures.

\section{Portability plan for ALTRAN-F}

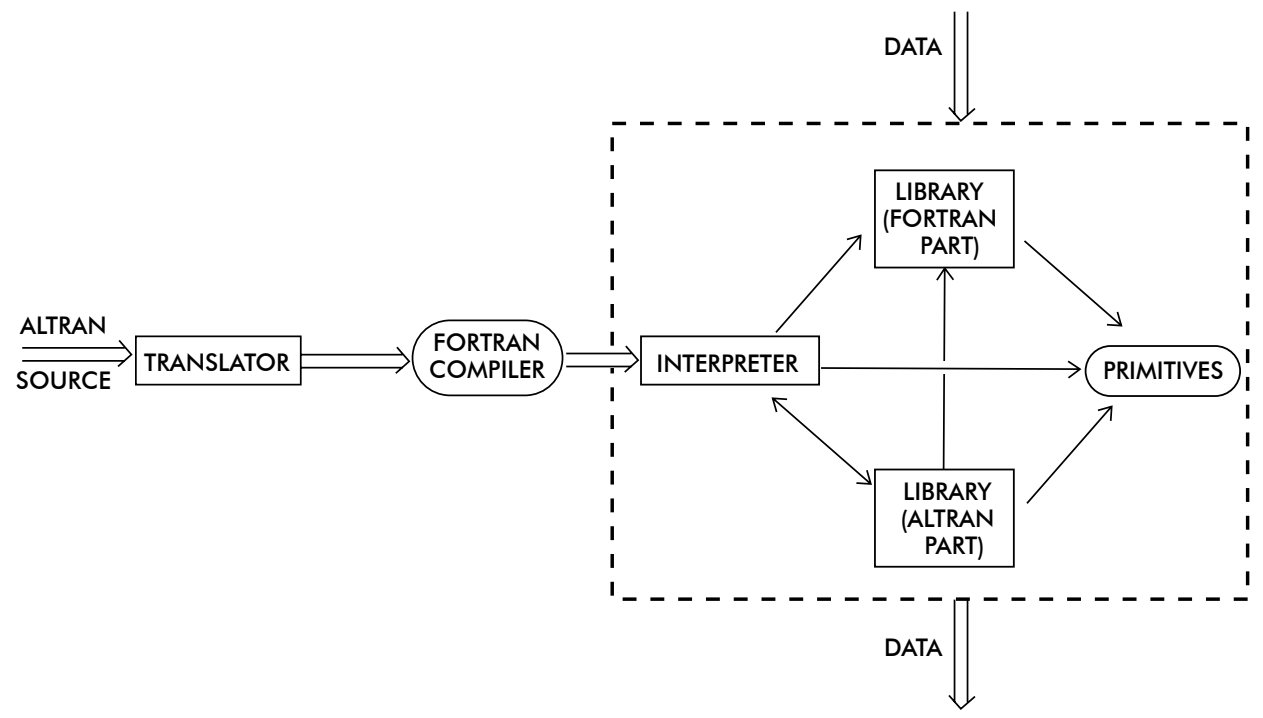

Figure 1. Overall structure of the system

* Because of this reliance on FORTRAN, the translator is not a compiler in the sense of class 3 above; hence the entire ALTRAN system fits into class 2. 
ALTRAN is a FORTRAN-like language incorporating certain symbolic algebraic data types and operations on data of these types. A new version called ALTRAN-F is now under construction, and is intended to be portable. The overall structure of the system is illustrated in Figure 1. The translator translates ALTRAN source into FORTRAN source, which can then be compiled by a FORTRAN compiler.* The resulting object deck consists 82 primarily of an encoding of the source program into a highly compact intermediate form. Identifiers are replaced by code numbers and their names and attributes are stored in a symbol table. The intermediate text and the symbol table are represented as integer arrays, and are decoded by the interpreter. Algebraic operations are performed by library routines - some written in FORTRAN and others in ALTRAN. A few machine language primitives, written separately for each installation, are also required.

The implementation language is a carefully defined subset of FORTRAN IV. A program unit is composed of (up to) seven parts in a prescribed order. Some statement types have been excluded, while others have been restricted. This language is supported by the standard FORTRAN compilers on the IBM 360, GE 625/635, CDC 6400/6600 and UNIVAC 1108 computers. To escape from its limitations, both macros and primitive subroutines are used. Macros permit extensions of the implementation language, while primitives allow for the efficient coding of critical operations.

A macro processor called M6 (see Reference 2) is included as a part of the ALTRAN system. This processor is written in the FORTRAN IV subset discussed above. The ALTRAN translator and interpreter and the FORTRAN part of the ALTRAN library are written in a machine independent macro extension of this subset. When these modules are processed by M6, the output is pure FORTRAN (in the subset), but is no longer machine independent. Certain macro definitions must, of course, be supplied separately for each installation.

The primary use of macros is to permit the introduction of machine and implementation parameters into the language. For example the number of characters per machine word is represented as a macro, to be used in FORMAT statements, DATA statements and the manipulation of character strings. The relative sizes of different data types are important for the proper use of EQUIVALENCE and COMMON. Implementation parameters such as the size of the work space, the length of an identifier and the unit numbers for various data streams are also represented as macros. Similarly macros permit the use of nonstandard types such as INTEGER $* 2$ on the 360 . Finally, macros permit the easy insertion and deletion of optional debugging 83 statements, and the macro calls that separate decks on the system tape can be used to generate control cards.

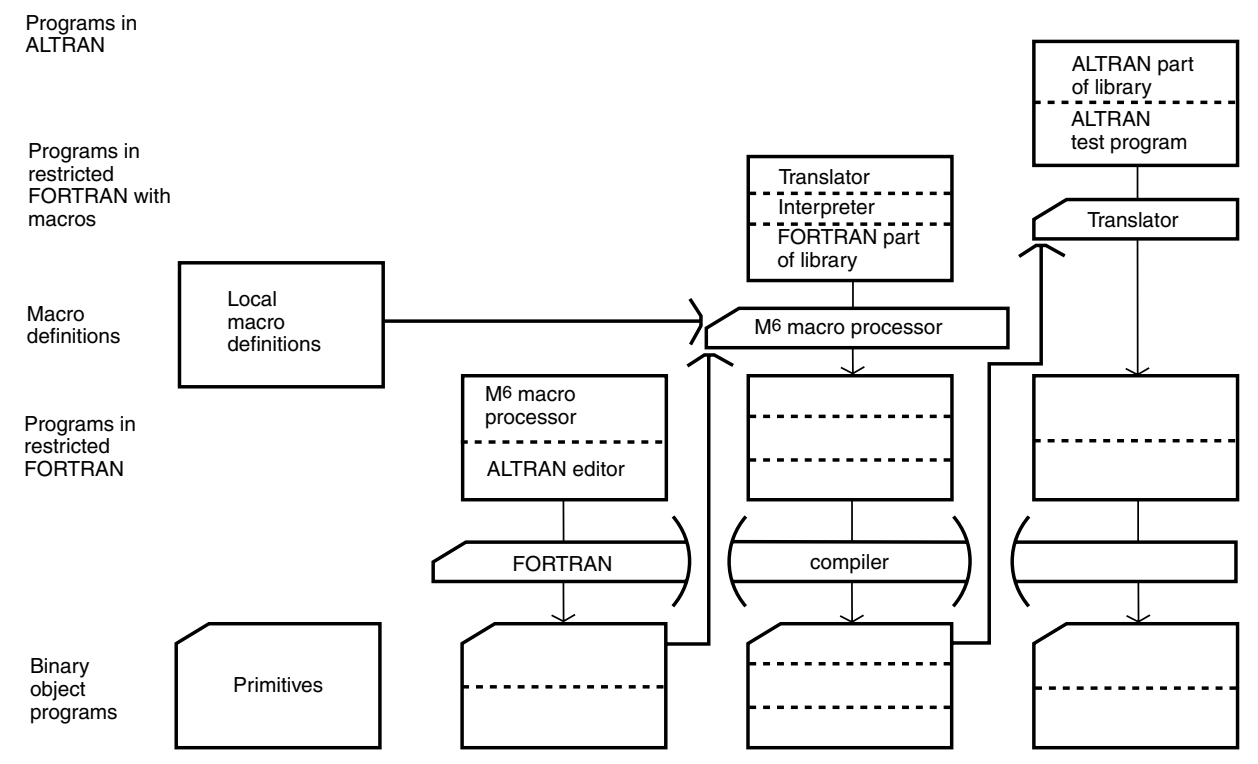

Figure 2. Installation steps

Primitives are used for the most elementary operations on strings, integers, reals and packed exponent sets. They are also used for the association of procedure names with entry addresses. 
The complete system consists of a system tape and some associated documentation. Manuals will be provided to describe the definition, implementation and use of the language. Another manual will discuss the procedures for installing, improving and maintaining the system.

The system tape consists of six logical files. The first contains the ALTRAN character set and the second contains an editor to be used for incorporating system updates. The third file contains the macro processor, M6 in source language and the fourth contains some basic macro definitions. Finally, the last two files contain the FORTRAN and ALTRAN parts of the system, respectively.

To install the system (see Figure 2), a recipient must first write some basic macros and some primitives, and transliterate the system tape into his local character set. Next he compiles the macro processor using his local FORTRAN compiler. Then he compiles the translator, the interpreter, and the FORTRAN part of the library, using the macro processor followed by the FORTRAN compiler. Then he compiles the ALTRAN part of the library, using the ALTRAN translator and the FORTRAN compiler. Finally he compiles and runs an ALTRAN test program to be sure that everything is in working order.

Once the system is operational, it can be improved by replacing critical procedures with more efficient machine language versions. These replacements can be made at leisure and in an order determined by local experience. However, the documentation indicates procedures that are known to be critical.

Improvements will be distributed occasionally in the form of an update letter. The changes can be keypunched from the update letter and incorporated with the aid of the editor. The editing technique has been designed in such a way that unrelated local modifications need not interfere with this process.

\section{4}

References

1. Brown, W. S. The ALTRAN language for symbolic algebra, unpublished, January 1969, pp. 84.

2. Hall, A. D. The $M^{6}$ macro processor; (unpublished), March 1969, pp. 16, 1 appendix. M6 was designed by M. D. McIlroy, using ideas from many sources. The FORTRAN implementation is a translation by A. D. Hall of an original written in MAD by R. Morris.

\subsection{Structured programming}

by

E. W. Dijkstra

\section{Introduction}

This working document reports on experience and insights gained in programming experiments performed by the author in the last year. The leading question was if it was conceivable to increase our programming ability by an order of magnitude and what techniques (mental, organizational or mechanical) could be applied in the process of program composition to produce this increase. The programming experiments were undertaken to shed light upon these questions.

\section{Program size}

My real concern is with intrinsically large programs. By "intrinsically large" I mean programs that are large due to the complexity of their task, in contrast to programs that have exploded (by inadequacy of the equipment, unhappy decisions, poor understanding of the problem, etc.). The fact that, for practical reasons, my experiments had thus far to be carried out with rather small programs did present a serious difficulty; I have tried to overcome this by treating problems of size explicitly and by trying to find their consequences as much as possible by analysis, inspection and reflection rather than by (as yet too expensive) experiments.

In doing so I found a number of subgoals that, apparently, we have to learn to achieve (if we don't already know how to do that). 
If a large program is a composition of $\mathrm{N}$ "program components", the confidence level of the individual components must be exceptionally high if $\mathrm{N}$ is very large. If the individual components can be made with the probability "p" of being correct, the probability that the whole program functions properly will not exceed

$$
\mathrm{P}=\mathrm{P}^{\mathrm{N}}
$$

for large $\mathrm{N}, \mathrm{p}$ must be practically equal to one if $\mathrm{P}$ is to differ significantly from zero. Combining subsets into larger components from which then the whole program is composed, presents no remedy:

$$
\mathrm{p}^{\mathrm{N} / 2 *} \mathrm{p}^{\mathrm{N} / 2} \text { still equals } \mathrm{p}^{\mathrm{N}} \text { ! }
$$

As a consequence, the problem of program correctness (confidence level) was one of my primary concerns.

The effort—be it intellectual or experimental — needed to demonstrate the correctness of a program in a sufficiently convincing manner may (measured in some loose sense) not grow more rapidly than in proportion to the program length (measured in an equally loose sense). If, for instance, the 85 labour involved in verifying the correct composition of a whole program out of $\mathrm{N}$ program components (each of them individually assumed to be correct) still grows exponentially with N. we had better admit defeat. Any large program will exist during its life-time in a multitude of different versions, so that in composing a large program we are not so much concerned with a single program, but with a whole family of related programs, containing alternative programs for the same job and/or similar programs for similar jobs. A program therefore should be conceived and understood as a member of a family; it should be so structured out of components that various members of this family, sharing components, do not only share the correctness demonstration of the shared components but also of the shared substructure.

\section{Program correctness}

An assertion of program correctness is an assertion about the net effects of the computations that may be evoked by this program. Investigating how such assertions can be justified, I came to the following conclusions:

1 The number of different inputs, i.e. the number of different computations for which the assertions claim to hold is so fantastically high that demonstration of correctness by sampling is completely out of the question. Program testing can be used to show the presence of bugs, but never to show their absence! Therefore, proof of program correctness should depend only upon the program text.

2 A number of people have shown that program correctness can be proved. Highly formal correctness proofs have been given; also correctness proofs have been given for "normal programs", i.e. programs not written with a proof procedure in mind. As is to be expected (and nobody is to be blamed for that) the circulating examples are concerned with rather small programs and, unless measures are taken, the amount of labour involved in proving might well (will) explode with program size.

3 Therefore, I have not focused my attention on the question "how do we prove the correctness of a given program?" but on the questions "for what program structures can we give correctness proofs without undue labour, even if the programs get large?" and, as a sequel, "how do we make, for a given task, such a well-structured program?". My willingness to confine my attention to such "well-structured programs" (as a subset of the set of all possible programs) is based on my belief that we can find such a well structured subset satisfying our programming needs, i.e. that for each programmable task this subset contains enough realistic programs.

4 This, what I call "constructive approach to the problem of program correctness", can be taken a step further. It is not restricted to general considerations as to what program structures are attractive from the point of view of provability; in a number of specific, very difficult programming tasks I have finally succeeded in constructing a program by analyzing how a proof could be given that a class of computations would satisfy certain requirements; from the requirements of the proof the program followed.

\section{The relation between program and computation}

Investigating how assertions about the possible computations (evolving in time) can be made on account of the static program text, I have concluded that adherence to rigid sequencing disciplines is essential, so as to allow step-wise abstraction from the possibly different routing. In particular: when programs for a sequential computer are expressed as a linear sequence of basic symbols of a programming language, sequencing should be 86 controlled by alterna- 
tive, conditional and repetitive clauses and procedure calls, rather than by statements transferring control to labelled points.

The need for step-wise abstraction from local sequencing is perhaps most convincingly shown by the following demonstration:

Let us consider a "stretched" program of the form

$$
\mathrm{S}_{1} ; \mathrm{S}_{2} ; \ldots ; \mathrm{S}_{\mathrm{N}}
$$

and let us introduce the measuring convention that when the net effect of the execution of each individual statement $\mathrm{S}_{\mathrm{j}}$ has been given, it takes $\mathrm{N}$ steps of reasoning to establish the correctness of program (1), i.e. to establish that the cumulative net effect of the $\mathrm{N}$ actions in succession satisfies the requirements imposed upon the computations evoked by program (1).

For a statement of the form

$$
\text { if } B \text { then } S_{1} \text { else } S_{2}
$$

where, again, the net effect of the execution of the constituent statements $S_{1}$ and $S_{2}$ has been given; we introduce the measuring convention that it takes 2 steps of reasoning to establish the net effect of program (2), viz. one for the case $\mathrm{B}$ and one for the case not $\mathrm{B}$.

Consider now a program of the form

$$
\begin{aligned}
& \text { if } B_{1} \text { then } S_{11} \text { else } S_{12} \text {; } \\
& \text { if } B_{2} \text { then } S_{21} \text { else } S_{22} \text {; } \\
& \text { if } B_{N} \text { then } S_{N 1} \text { else } S_{N 2}
\end{aligned}
$$

According to the measuring convention it takes 2 steps per alternative statement to understand it, i.e. to establish that the net effect of

$$
\text { if } B_{i} \text { then } S_{i 1} \text { else } S_{i 2}
$$

is equivalent to that of the execution of an abstract statement $\mathrm{S}_{1}$. Having $\mathrm{N}$ such alternative statements, it takes us $2 \mathrm{~N}$ steps to reduce program(3) to one of the form of program (1); to understand the latter form of the program takes us another $\mathrm{N}$ steps, giving $3 \mathrm{~N}$ steps in toto.

If we had refused to introduce the abstract statements $S_{i}$ but had tried to understand program (3) directly in terms of executions of the statements $S_{\mathrm{ij}}$, each such computation would be the cumulative effect of $\mathrm{N}$ such statement executions and would as such require $\mathrm{N}$ steps to understand it. Trying to understand the algorithm in terms of the $\mathrm{S}_{\mathrm{ij}}$ implies that we have to distinguish between $2 \mathrm{~N}$ different routings through the program and this would lead to $\mathrm{N} * 2 \mathrm{~N}$ steps of reasoning!

I trust that the above calculation convincingly demonstrates the need for the introduction of the abstract statements $\mathrm{S}_{\mathrm{i}}$. An aspect of my constructive approach is not to reduce a given program (3) to an abstract program (1), but to start with the latter.

\section{Abstract data structures}

Understanding a program composed from a modest number of abstract statements again becomes an exploding task if the definition of the net effect of the constituent statements is sufficiently unwieldy. This can be overcome by the introduction of suitable abstract data structures. The situation is greatly analogous to the way in which we can understand an ALGOL program operating on integers without having to bother about the number representation of the 
implementation used. The only difference is that now the programmer must invent his own concepts (analogous to the "ready-made" integer) and his own operations upon them (analogous to the "ready-made" arithmetic operations).

87

In the refinement of an abstract program (i.e. composed from abstract statements operating on abstract data structures) we observe the phenomenon of "joint refinement". For abstract data structures of a given type a certain representation is chosen in terms of new (perhaps still rather abstract) data structures. The immediate consequence of this design decision is that the abstract statements operating upon the original abstract data structure have to be redefined in terms of algorithmic refinements operating upon the new data structures in terms of which it was decided to represent the original abstract data structure. Such a joint refinement of data structure and associated statements should be an isolated unit of the program text: it embodies the immediate consequences of an (independent) design decision and is as such the natural unit of interchange for program modification. It is an example of what I have grown into calling "a pearl".

\section{Programs as necklaces strung from pearls}

I have grown to regard a program as an ordered set of pearls, a "necklace". The top pearl describes the program in its most abstract form, in all lower pearls one or more concepts used above are explained (refined) in terms of concepts to be explained (refined) in pearls below it, while the bottom pearl eventually explains what still has to be explained in terms of a standard interface (=machine). The pearl seems to be a natural program module.

As each pearl embodies a specific design decision (or, as the case may be, a specific aspect of the original problem statement) it is the natural unit of interchange in program modification (or, as the case may be, program adaptation to a change in problem statement).

Pearls and necklace give a clear status to an "incomplete program", consisting of the top half of a necklace; it can be regarded as a complete program to be executed by a suitable machine (of which the bottom half of the necklace gives a feasible implementation). As such, the correctness of the upper half of the necklace can be established regardless of the choice of the bottom half.

Between two successive pearls we can make a "cut", which is a manual for a machine provided by the part of the necklace below the cut and used by the program represented by the part of the necklace above the cut. This manual serves as an interface between the two parts of the necklace. We feel this form of interface more helpful than regarding data representation as an interface between operations, in particular more helpful towards ensuring the combinatorial freedom required for program adaptation.

The combinatorial freedom just mentioned seems to be the only way in which we can make a program as part of a family or "in many (potential) versions" without the labour involved increasing proportional to the number of members of the family. The family becomes the set of those selections from a given collection of pearls that can be strung into a fitting necklace.

\section{Concluding remarks}

Pearls in a necklace have a strict logical order, say "from top to bottom". I would like to stress that this order may be radically different from the order (in time) in which they are designed.

Pearls have emerged as program modules when I tried to map upon each other as completely as possible, the numerous members of a class of related programs. The abstraction process involved in this mapping turns out (not, amazingly, as an afterthought!) to be the same as the one that can be used to reduce the amount of intellectual labour involved in correctness proofs. This is very encouraging.

As I said before, the programming experiments have been carried out 88 with relatively small programs. Although, personally, I firmly believe that they show the way towards more reliable composition of really large programs, I should like to stress that as yet I have no experimental evidence for this. The experimental evidence gained so far shows an increasing ability to compose programs of the size I tried. Although I tried to do it, I feel that I have given but little recognition to the requirements of program development such as is needed when one wishes to employ a large crowd; I have no experience with the Chinese Army approach, nor am I convinced of its virtues.

\section{NATO SOFTWARE ENGINEERING CONFERENCE 1969}




\subsection{Criteria for a system design language}

by

A. D. Falkoff

A system is a collection of programs and a program is a formal statement of an algorithm or a process. This paper proposes that the appropriate design medium for systems is a formal programming language.

Since the programs that comprise a system may be implemented in various gradations of hardware and software, the proposal is not restricted to software, although it is the software design problem that is of interest at the moment. Indeed, the unifying effect of using a common medium and the flexibility it provides in the timing of implementation decisions are two of the major advantages to be expected.

In proposing the use of a programming language in system design it is not intended to outlaw the use of English or other natural languages, either in the formal documentation that should be part of a design effort, or in the casual communication that always occurs in an engineering operation. Rather, the use of the two types of languages should be complementary. The natural language can be used to convey broad ideas quickly, while the formal language can be used to make these ideas precise and ultimately to act as a guide in preparing natural language documentation, such as manuals.

The criteria that are proposed for the choice of a formal design language are:

1 It should be easy to learn its basic use.

2 Formulations in the language should be suggestive and thought provoking.

3 It should allow meaningful formal manipulation of both expressions and larger program segments.

4 It should lead to the development of aesthetic criteria for judging the quality of a design.

$5 \quad$ It should be convenient for documentation.

$6 \quad$ It should be uncommitted to any particular technology or problem area.

$7 \quad$ It should be applicable in a consistent way to systems of any foreseeable complexity.

8 It should be executable on a machine.

Each of these criteria will be briefly discussed below, with some examples in terms of APL (see References 1, 2 and $3)$.

1 Because of the continual problem of training new designers and the immediate perturbation when working designers start to use it, the basic use of a design language should be easy to learn. That is to say, the beginner should be able to produce correct and usable programs in a 89 relatively short time, while power and elegance in the use of the language come with practice.

This requirement suggests that the language should have a syntax and semantics built up from a small number of internally consistent rules. Syntactical restrictions and semantic surprises of the kind that have arisen when convenience of implementation was taken as a significant guide in language design must be avoided.

2 Apart from clerical tasks, good design requires thought. A language which is awkward to use or which suppresses all detail will, in the first instance, discourage experimentation with different formulations and, in the second instance, fail to suggest alternative lines of attack. This suggests that, while the design language should have a simple structure, it should at the same time have a rich set of primitive operations so that, even at fairly high levels of design, actions can be expressed succinctly in terms of primitive operations.

For example, Figure 1 gives two systems of skeletal programs for maintaining a table. The APPEND-DELETE system maintains LST by appending to it and contracting it, as required. The INSERT-ERASE system maintains TBL, conceived as a fixed length object, by keeping a dynamic record of the next available space. This record also serves in a complementary fashion to identify valid table entries for readout. The two systems were obviously easy to write and are not very hard to understand. Which would be better in a given environment, or would a combination of the two be best? 


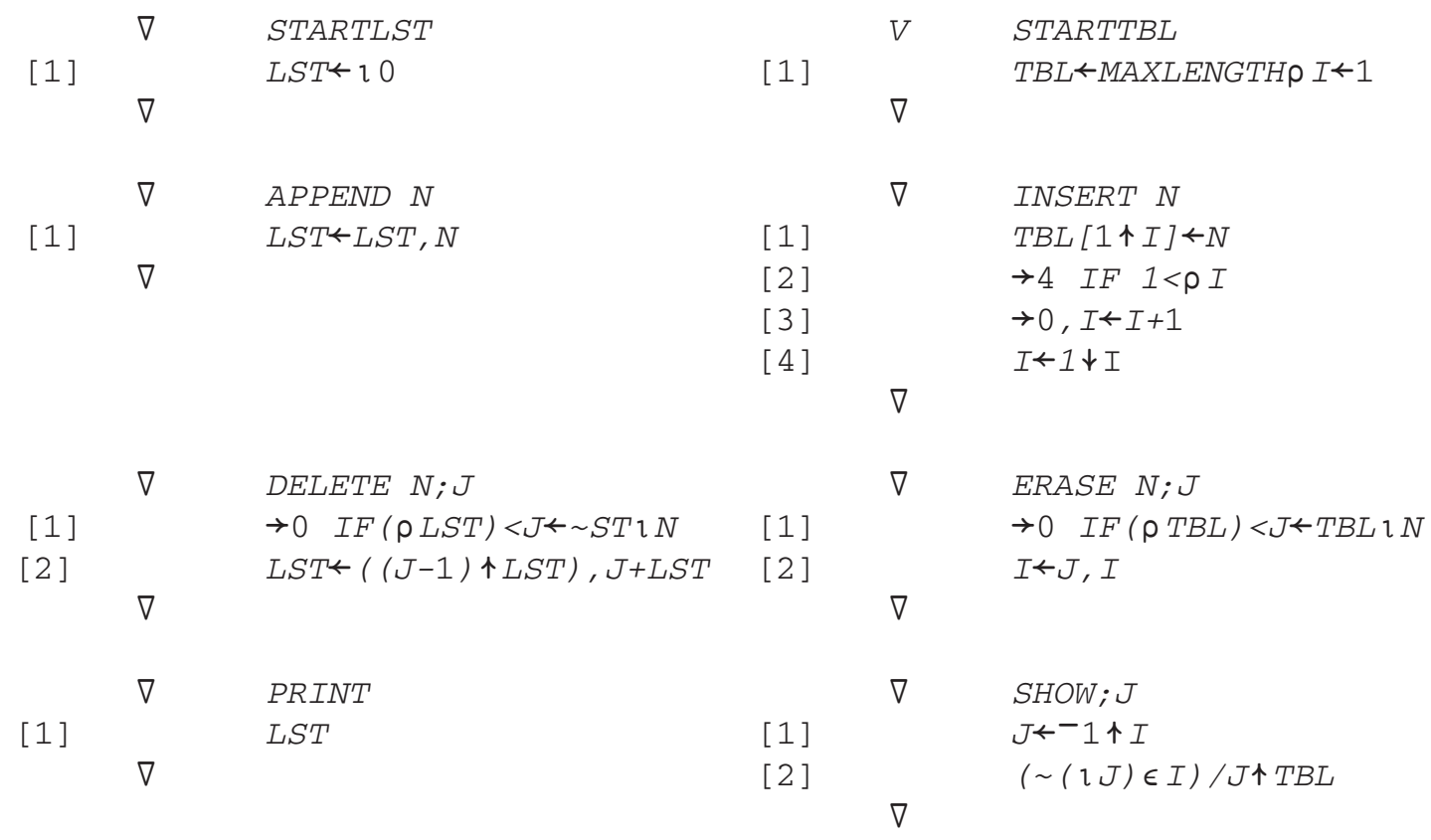

Figure I. Two systems for maintaining a table

3 One aspect of formal manipulation is that it opens a way to finding proofs of equivalence among programs, an acknowledged problem in system design and implementation. A more immediately practical aspect of it, however, is one related to the second criterion. That is, formal manipulation provides a quasi-mechanical way to search for equivalent formulations that are in some sense better than others.

\section{0}

The exploitation of complete formal equivalence has been treated at length in a general context by Iverson (see Reference 4) and an instance of its use in hardware design has been shown by Falkoff (see Reference 5).

Substitution of nearly equivalent forms can bring to light, by their connotations, facets of problems that might otherwise be overlooked. Thus it is clear that the following formulations for the selection of an element from a vector are equivalent, over the range of possible indices of the element, and that they are mechanically derivable one from another:

$Q+T B L[P]$ (indexing)

$R \leftarrow(($ I $\rho T B L) \in P) / T B L$ (compression by the characteristic vector of $P$ over the set of indices of $T B L)$

$S \leftarrow^{-} 1 \uparrow P \uparrow T B L \quad$ (select the last of the first $P$ elements of $T B L$ )

$T \leftarrow^{-} 1 \uparrow P \rho T B L \quad$ (select the last of the $P$ replications of the elements of $T B L$ )

If $P$ is outside the index range: $Q$ is never specified and an error is reported; $R$ will be an empty vector; $S$ will be set to zero, the default action provided for on the exceeding of a vector bound; and $T$ will be the element reached by endaround addressing. Stating the last case formally it can be seen that the circle is closed in two senses: $-1+P \rho T B L$ is completely equivalent to $T B L[(\rho T B L) I P]$ (in zero-origin). Which of the foregoing results is best in a given situation is, of course, a moot question but the formal manipulation makes it evident that a question exists.

To allow effective formal manipulation a language should have a syntax that is context-free, a semantics that is free of side-effects and a well chosen set of primitive operations. A graphic quality is helpful, if not essential, for the treatment of program segments larger than individual statements. 
4 In engineering or architectural design, aesthetic criteria are the ultimate basis for design decisions that are not dictated by strictly technical considerations. Good designers are distinguished from others by their exercise of aesthetic judgment. A good design language should therefore promote the development of such criteria. This means that it should be possible both to comprehend the total structure of a system in some graphic fashion and to examine details, as required. If a language has this property designers will develop both conscious and intuitive bases for judging the symmetry, balance and general rightness for its purpose of a proposed design.

Consider the program in Figure 2, which is a description of the channel behaviour of System 360, taken from Reference 2. The area blocked out in a heavy line is the part of the program in which data transfer actually takes place between a peripheral device and the central memory. The other parts of the program perform control functions of one kind or another. On the face of it, is this a reasonable balance? The answer, at this time, is not known but a good design tool should provoke questions of this kind.

To provide such a facility, a formal language should clearly be amenable to graphic display. But more significantly, it should have a simple structure so that it does not burden the design with its own complexity. It should be sufficiently rich in primitive operations to permit reasonably complex substructures to be displayed directly, so that the heavy use of defined functions becomes a measure of what is actually being swept under the carpet, rather than an enforced way of life.

91

5 The need for convenience in documentation need not be laboured. Two things are meant by convenience in this context. One is that primary documents should come naturally out of the design process as is the case, for example, with mechanical parts drawings. The other is that it should not require massive equipment or microscopic records to document large systems.

The first purpose could be served by almost any programming language, if properly used, and its pursuit will lead naturally to consideration of work organization and administrative procedures. The second purpose is best served by a language that permits terse constructions both within statements and in the display of program sequences.

6 Because programming is a tool in so many fields, a formal design language will be enhanced in usefulness if it is not biased toward any particular field. The operation of a computing machine is only one instance of the application of programming; the design of the machine itself is another; and certain aspects of its manufacture are others. In particular, because of the hazy division between hardware and software, it is important that a system design language be not biased in favour of one or the other.

A bias toward a particular field is most often manifested by the incorporation in the language of specialized data objects (e.g. pointers), by the pre-assignment of functional significance to otherwise abstract structures (e.g. 101 taken as the number five rather than a vector of three elements) and by a preoccupation with the representations of numbers and characters. Since considerations of this nature can be conveniently handled by functions defined on abstract objects that are at home in any field, a design language can be free of such bias. But, as a corollary, a design language must have a strong function-definition capability. It should be possible to define easily functions on the primitive objects of the language and to use them without syntactic distinction from the primitive functions provided by the language.

7 In a general way, system complexity is characterized by the degree to which different programs in a system interact. Such interaction seems to take two basic forms: the exercise of control over one program by another, and the use of common variables by different programs.

For the exercise of control, the ability to use one program in another (which is inherent in the notion of a defined function) together with the ability of one program to alter explicitly the execution sequence of an otherwise independent program seems to be sufficient. The use of common variables requires only the conventions necessary to establish that they are common (or, conversely, that some variables are not). Reference 2 is an example of a complex system, with many interactions among programs, in which these devices were sufficient.

8 Like documentation, the need for machine testing of designs requires no argument. It need be noted only that a formal language capable of expressing the design of a system at different levels of detail can be used directly for simulating the system at any design stage. It is necessary only to substitute appropriate tables for functions that are not yet detailed.Machinability also provides the opportunity to have "live" catalogues on interactive systems, in which functional units can be displayed and executed for gaining understanding of their behaviour.

\section{NATO SOFTWARE ENGINEERING CONFERENCE 1969}


92

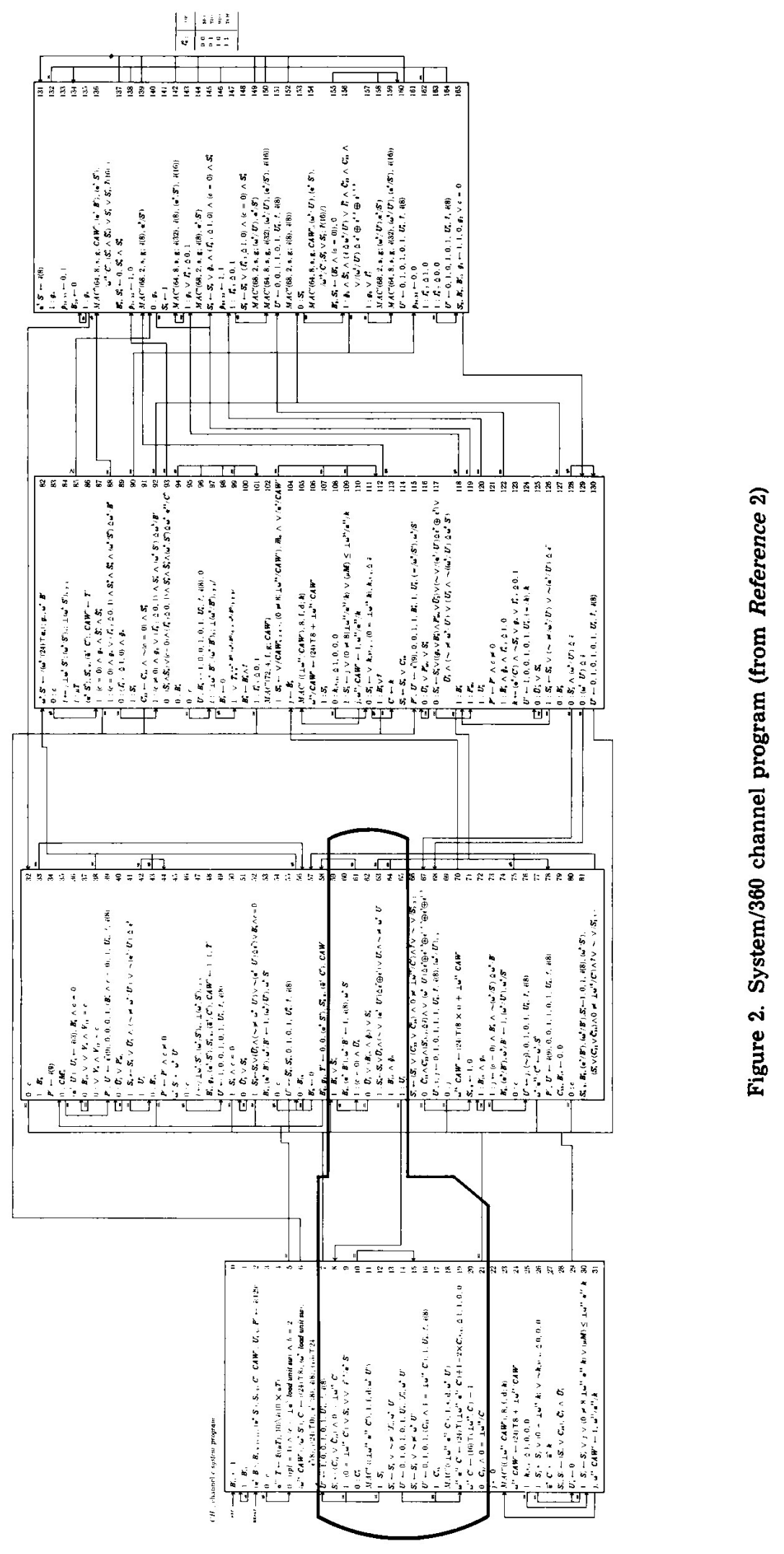


In conclusion it is worth noting a significant aspect of the use of a formal language in system design. Namely, any formal specification can be viewed either as a function which specifies certain transformations or as a set of instructions for executing these transformations. The choice of viewpoint is the prerogative and obligation of the user. It is, in fact, one of the 93 advantages of the use of formal specification in design that, even though the formulator may write a program with the intent that it be literally followed in some more detailed representation, it is nevertheless also a functional specification, and the perceptive designer will see it in this light and perhaps be able (because it is also well defined) to find an equivalent formulation that has greater economy or other engineering advantages.

References

1. Iverson, K. E. A Programming Language, Wiley, (New York), 1962.

2. Falkoff, A. D., Iverson, K. E. and Sussenguth, E. H. A formal description of system 360, IBM Systems Journal, 3, No. 3, 1964.

3. Falkoff, A. D. and Iverson, K. E. APL 360 User's Manual, IBM Research (Yorktown Hts., New York), 1968.

4. Iverson, K. E. Formalism in programming languages, $C A C M$, 7, 1964, pp. 80-88.

5. Falkoff A. D. Algorithms for parallel-search memories, JACM, 9, 1962, pp. 488-511.

93

\subsection{System evaluation tools \\ by \\ C. C. Gotlieb and G. H. MacEwen}

\section{Classification of tools}

At the first NATO Conference on Software Engineering Pinkerton (see Reference 1) listed techniques for the collection of system performance data and discussed some of the uses of such data. Data collection, or monitoring, may be regarded as one of two basic tools useful for system design, the other being modelling. Each of these basic tools, modelling and monitoring, may be further subdivided into two types yielding four kinds of system evaluation tools, as follows:

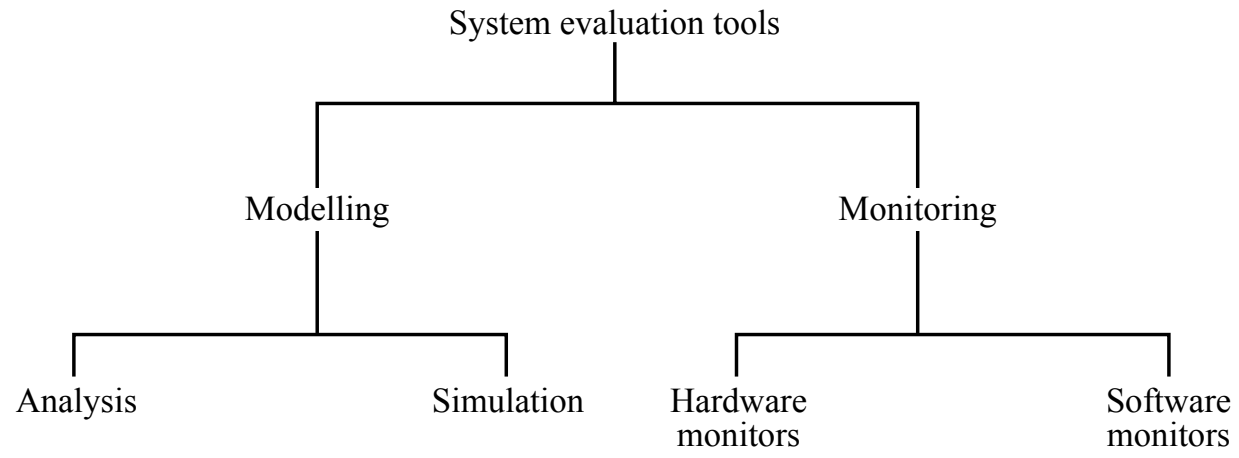

\section{Applicability of tools and comparisons}

Very broadly we have three phases of system design: conceptional, construction and operational. On studying or designing a system it is possible to bring more than one of the above tools into play. Each is particularly useful during different phases and the results can reinforce each other. In general modelling is most useful during the conceptional and construction phases, while monitoring is most useful in the construction and operational phases.

\section{4}

Figure 1 illustrates in simplified form the interaction between the tools and the system. Briefly:

- $\quad$ models predict performance and monitors measure it.

- $\quad$ models are useful in determining which quantities should be monitored.

- $\quad$ monitors can be used to determine which quantities should be designated as design parameters. 


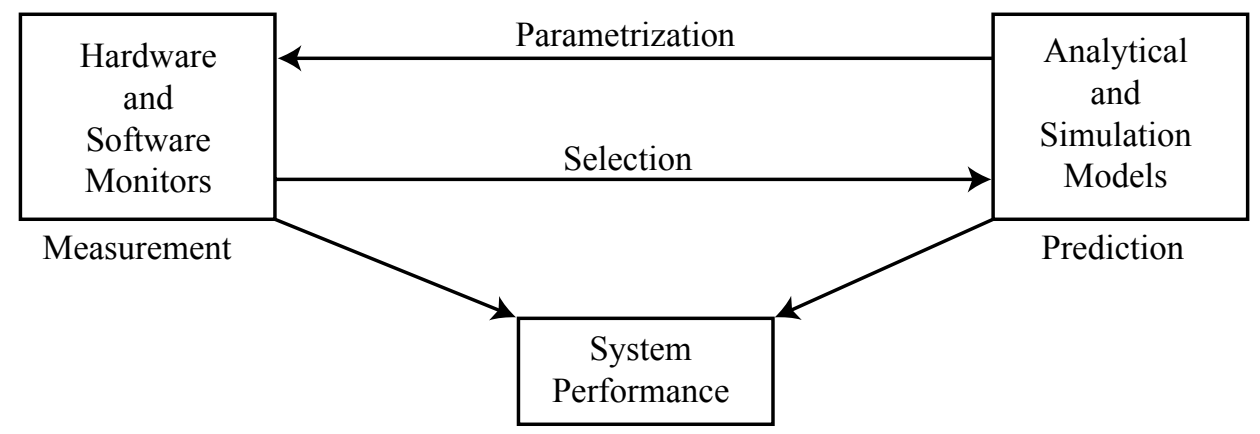

A more detailed comparison of the tools and their range of use is given in Table 1.

\section{Some implementations}

Analytical models have been used extensively to study the behaviour of computer systems where queueing plays an important role, e.g. in time sharing systems (see References 2 and 3) or in job shop scheduling (see Reference 4). They have also been successful in characterizing the behaviour of a particular hardware or software component, e.g. a disc memory (see Reference 5), or a system of addressing (see Reference 6).

General purpose simulators have been used for some time. At least two, GPSS (see Reference 7) and SIMSCRIPT (see Reference 8), have very wide currency and many of the computer system studies reported in the Second Conference on Applications of Simulation (see Reference 9) are based on them (see for example Frank (see Reference 10)). Another type of computer system simulation takes as input a description of the hardware corresponding to a configuration (peripheral devices, controllers and channels, memory banks, CPU) and also specifications of the operating system. When a proposed configuration and load are input, the activity is simulated and measures of how much system resources are needed to handle the load are produced. Examples of this type of simulator are S3 and CASE (see References $9 \mathrm{a}$ and $9 \mathrm{~b}$ ), a system available from the Computer Learning and Systems Corporation. A number of special purpose simulators are also described in the Conference Digest referenced above and elsewhere in the literature (see for example Reference 11).

Although some hardware monitors are described in the general literature (see for example Estrin Reference 12, and Schulman Reference 13), until recently most were developed by manufacturers in the process of their system design, or as an aid for analyzing a special configuration of some customer. Monitors of this type are now available commercially and as well there are software monitors developed by manufacturers or available commercially. Table 2 lists some monitors, hardware and software, currently available, with comments on the information they yield.

\section{8}

\section{Some possible directions}

The list of monitors and models given in the table and in the references is by no means a complete list of those generally available. And it is likely that there have been more of these types of tools developed by computer manufacturers for their own design, than have been described in the general literature. There would still seem to be some useful direction of exploration for these tools. Examples are:

- $\quad$ very little has been reported on the consistency of the results that might be found on using different tools on the same system.

- it would be useful to have specific guidelines for knowing when to use which tool.

- models or simulators should be developed so that they can accept as input descriptions of configurations that are not tied to the devices of a particular manufacturer. The components should have key parameters specified to the extent that will allow effective prediction of performance. For example there might be a memory hierarchy, where for each level of memory (high speed core, low speed core, drum, disc and tape) the access time, latency, memory size, etc. are specified. The tool should be able to yield information on how the storage transfers and memory overlays should take place. The information might be in the form 
Table 1 - Comparison of system evaluation tools

\begin{tabular}{|c|c|c|c|c|}
\hline & \multicolumn{2}{|c|}{ Models } & \multicolumn{2}{|c|}{ Monitors } \\
\hline & Analytical & Simulation & Software & Hardware \\
\hline \multirow[t]{2}{*}{ Required input } & \multicolumn{2}{|c|}{ Model of system under test } & \multicolumn{2}{|c|}{ Functioning system } \\
\hline & $\begin{array}{l}\text { Knowledge of hard- } \\
\text { ware and software } \\
\text { Mathematical tools } \\
\text { e.g. statistics, } \\
\text { queueing theory, etc }\end{array}$ & $\begin{array}{l}\text { Simulation package or } \\
\text { special simulator }\end{array}$ & $\begin{array}{l}\text { Software monitor } \\
\text { program }\end{array}$ & $\begin{array}{l}\text { Hardware monitor } \\
\text { device }\end{array}$ \\
\hline $\begin{array}{l}\text { Range of } \\
\text { application }\end{array}$ & \multicolumn{2}{|c|}{ Design, construction } & \multicolumn{2}{|c|}{ Construction, operations, optimization } \\
\hline \multirow[t]{3}{*}{ Results } & \multicolumn{2}{|c|}{ Performance data } & \multicolumn{2}{|c|}{ Utilization of resources } \\
\hline & $\begin{array}{l}\text { For a wide range } \\
\text { of parameters } \\
\text { Dependence of per }\end{array}$ & $\begin{array}{l}\text { For specified sets } \\
\text { of parameters } \\
\text { rmance on parameters }\end{array}$ & $\begin{array}{l}\text { Software and } \\
\text { hardware } \\
\text { Statistics gathe }\end{array}$ & $\begin{array}{l}\text { Hardware } \\
\text { ered may be }\end{array}$ \\
\hline & Calculated & Displayed & $\begin{array}{l}\text { in any form, e.g. } \\
\text { counts, durations, } \\
\text { mean values, histo- } \\
\text { grams, etc. }\end{array}$ & $\begin{array}{l}\text { special form, e.g. } \\
\text { counts of events, total } \\
\text { time duration of } \\
\text { some condition of } \\
\text { logical combination } \\
\text { of conditions }\end{array}$ \\
\hline \multirow{2}{*}{$\begin{array}{l}\text { Validity of } \\
\text { results }\end{array}$} & \multicolumn{2}{|c|}{ Very dependent on model } & \multirow{2}{*}{\multicolumn{2}{|c|}{$\begin{array}{l}\text { Very dependent on work load during testing } \\
\text { e.g. job mix, load level, etc. }\end{array}$}} \\
\hline & $\begin{array}{l}\text { Accuracy need not } \\
\text { have statistical } \\
\text { dependence }\end{array}$ & $\begin{array}{l}\text { Accuracy dependent } \\
\text { on run time of } \\
\text { simulation - results } \\
\text { invalid if steady } \\
\text { state not achieved }\end{array}$ & & \\
\hline Advantages & $\begin{array}{l}\text { Performance can be } \\
\text { determined over a } \\
\text { wide continuous } \\
\text { range of parameters. } \\
\text { Time dependent of } \\
\text { steady state } \\
\text { models possible }\end{array}$ & $\begin{array}{l}\text { Work load and con- } \\
\text { figuration - can } \\
\text { concentrate on parts } \\
\text { of system which are } \\
\text { bottle necks }\end{array}$ & $\begin{array}{l}\text { Can monitor hardware } \\
\text { and software perfor- } \\
\text { mance } \\
\text { Work best when monit } \\
\text { into system design }\end{array}$ & $\begin{array}{l}\text { Can monitor any } \\
\text { accessible hardware } \\
\text { function. Does not } \\
\text { alter system under } \\
\text { test } \\
\text { ors are incorporated }\end{array}$ \\
\hline Disadvantages & $\begin{array}{l}\text { Mathematics soon } \\
\text { become intractable } \\
\text { for all but simple } \\
\text { systems }\end{array}$ & $\begin{array}{l}\text { May be difficult to } \\
\text { model sufficient } \\
\text { detail of system and } \\
\text { maintain manageable } \\
\text { size of program. Must } \\
\text { be written and main- } \\
\text { tained for each re- } \\
\text { lease of each system }\end{array}$ & $\begin{array}{l}\text { Alters system under } \\
\text { test. Difficult to } \\
\text { make changes and } \\
\text { gather new data. } \\
\text { Must write and } \\
\text { maintain a monitor } \\
\text { for each release of } \\
\text { each system }\end{array}$ & $\begin{array}{l}\text { Cannot directly } \\
\text { measure software } \\
\text { functions, e.g. } \\
\text { queueing time must } \\
\text { be calculated by } \\
\text { supplementary } \\
\text { analysis }\end{array}$ \\
\hline
\end{tabular}


Table 2 - Some available monitors

\begin{tabular}{|c|c|c|c|}
\hline Name & Type & Supplier & Comments \\
\hline $\begin{array}{l}\text { Configuration } \\
\text { Utilization } \\
\text { Efficiency } \\
\text { Product (CUE) }\end{array}$ & $\begin{array}{l}\text { Software Mon. } \\
(\mathrm{OS} / 360)\end{array}$ & $\begin{array}{l}\text { Boole and Babbage } \\
\text { Inc. }\end{array}$ & $\begin{array}{l}\text { Measures total times for certain conditions, } \\
\text { e.g. CPU busy, channels busy, Cannot } \\
\text { measure times for logical combinations } \\
\text { of conditions. Gives count of SVC module } \\
\text { loads. Gives count and Track addresses of } \\
\text { disk seeks. }\end{array}$ \\
\hline MVTANAL & $\begin{array}{l}\text { Software Mon. } \\
\text { (OS/360/MVT) }\end{array}$ & $\begin{array}{l}\text { IBM (for use by } \\
\text { IBM only) }\end{array}$ & $\begin{array}{l}\text { Gives count of usage of OS routines. Mea- } \\
\text { sures usage of I/O devices and distribution } \\
\text { of queue length for each device. Measures } \\
\text { hardware utilization, e.g. CPU, channel. } \\
\text { Measures usage of system datasets }\end{array}$ \\
\hline $\begin{array}{l}\text { Problem Program } \\
\text { Efficiency (PPE) }\end{array}$ & $\begin{array}{l}\text { Software Mon. } \\
(\mathrm{OS} / 360)\end{array}$ & $\begin{array}{l}\text { Boole and Babbage } \\
\text { Inc. }\end{array}$ & $\begin{array}{l}\text { Used on applications and programs - } \\
\text { identifies portions of programs with high } \\
\text { usage, where I/O waits arise, etc. }\end{array}$ \\
\hline $\begin{array}{l}\text { System Utilization } \\
\text { Monitor (SUM) }\end{array}$ & Hardware Mon. & $\begin{array}{l}\text { Computer Synectics } \\
\text { Inc. }\end{array}$ & $\begin{array}{l}\text { Readings taken every millisecond and } \\
\text { stored on tape. Data reduction program } \\
\text { gives: (a) Total time that certain condi- } \\
\text { tions or logical combination of conditions } \\
\text { were active, e.g. CPU busy, channel busy } \\
\text { and CPU waiting. (b) Counts for certain } \\
\text { events, e.g. total number of disk seeks }\end{array}$ \\
\hline $\begin{array}{l}\text { Computer Performance } \\
\text { Monitor (CPM) }\end{array}$ & Hardware Mon. & $\begin{array}{l}\text { Allied Computer } \\
\text { Technology Inc. }\end{array}$ & Similar to SUM \\
\hline $\begin{array}{l}\text { Basic Counter Unit } \\
(\mathrm{BCU})\end{array}$ & $\begin{array}{l}\text { Hardware Mon. } \\
\text { (System/360, } \\
1800,1130)\end{array}$ & $\begin{array}{l}\text { IBM (for use by } \\
\text { IBM only) }\end{array}$ & $\begin{array}{l}\text { Readings are taken manually or punched on } \\
\text { cards. Output consists of the total time that } \\
\text { certain conditions or logical combination of } \\
\text { conditions were active }\end{array}$ \\
\hline
\end{tabular}

of a general strategy but even failing this it would be useful to know how a particular program should be structured, or even how the memory blocks should. be distributed on a particular disc. As seen above, such tools are useful enough generally to make them attractive possibilities for commercial development, but more needs to be done.

- more analysis is needed of devices that are coming to be used very heavily, for example: disc memories with multiple modules or with more than one channel, multiplexers as used in remote job entry systems, etc.

\section{References}

1. Pinkerton, T. B. Performance monitoring and systems evaluation. Software Engineering, NATO Scientific Affairs Division, Brussels 39, pp. 200-203.

2. Coffman, E. G. and Kleinrock, L. Feedback queueing models for time-shared systems, JACM 15, No. 4, October 1968, pp. 549-576.

3. Adiri, I. and Avi-ltzhak, B. A time-sharing queue with a finite number of customers, JACM, 16, No. 2, April 1969, pp. 315-323. 
4. Rolfson, C. B. Scheduling workflow in a computer system, Ph.D. thesis, Dept. of Computer Science, University of Toronto, 1968.

5. Abate, J., Dubner, H. and Weinberg, S. B. Queueing analysis of the IBM 2314 disc storage facility, JACM, 15, No. 4, October 1968, pp. 577-589.

6. Peterson, W. W. Addressing for random access storage, IBM Journal of Research \& Dev. 1,1957, pp. 130-146.

7. Herscovitch, H. and Schneider, T. GPSS III—An expanded general purpose simulator, IBM Systems Journal, 4, No. 3, 1965, pp. 174-183.

8. Kiviat, P. J. and Villanueva, R. The SIMSCRIPT II programming language, The RAND Corp. R-460-PR, 1968.

9. Digest of the second conference on applications of simulation sponsored by SHARE/ACM/IEEE/SCI, December 1968, New York.

9a. Cohen, I. J. S3, The system and software simulator, Digest of the second conference on applications of simulation sponsored by SHARE/ACM/IEEE/SCI, December 1968, New York, pp. 282-285.

9b. Thompson, W. C. The application of simulation in computer system design and optimization, Digest of the second conference on applications of simulation sponsored by SHARE/ACM/IEEE/SCI, December 1968, New York, pp. 286-290.

10. Frank, A. L. The use of simulation in the design of information systems, Digest of the second conference on applications of simulation sponsored by SHARE/ACM/IEEE/SCI, December 1968, New York, pp. 87-88.

11. Katz, J. H. System/360 simulator, Comm. ACM, 10, No. 11, November 1967, pp. 694-702.

12. Estrin, G. et al, SNUPER computer-a computer instrumentation automaton, Proceedings of the SJCC, 1967, pp. 645-656.

13 Schulman, F. D. Hardware measurement device for IBM S/360 time-sharing operation, Proceedings of the 22nd ACM Conference, 1967, pp. 103-109.

99

\subsection{Computer aided software design}

by

M. E. Hopkins

Performance is one of the main concerns of those designing and developing any large programming system. The great paradox is that by common consent too much effort is expended to obtain performance and yet our great failures are often the result of an inefficient system.

Designers generally recognize that high performance is usually obtained through managing logistics in an optimal manner rather than by clever code. Logistics here is taken to mean the organization and flow of data which is in tables and files. A designer will employ various strategies in organizing data to realize an efficient flow. Where the task is well understood, the environment is reasonably stable, and the application is not subject to new developments, the designer can make early, judicious decisions about data organization. A typical example of this is our ability to write all but the most complex compilers with a small effort compared to that which was required several years ago when compilation was not as well understood. One wonders whether history will be repeated with operating systems. The experience of the last few years should certainly provide understanding but it seems questionable whether increased understanding alone will be enough to cope efficiently with the wide range of hardware configurations and job mixes which a general purpose operating system must handle.

In designing a general purpose operating system or any large application, the complexity of the task and requirements, which only become apparent late in development or during maintenance, tend to vitiate early design decisions made to improve performance. At this point the designers must be able to restructure the data to obtain their goals. In the absence of such capability several courses will be taken.

1 New tables will be specified by the designers which, they hope, will provide faster access than the few central tables of the initial design. 
2 Multiple methods of access are created to improve information flow. This implies complex interconnection in the form of pointers, complex searching procedures, etc.

3 Implementers develop their own private information repositories which again provide economies not available in central tables under design control.

All of this is almost inevitable in a production situation but several iterations produce disastrous results. For example OS/360 has a manual of over 100 pages describing "the major control blocks and tables". A casual examination of components reveals that each module has many complex private tables which are not included among "the major control blocks and tables." For example the Job Scheduler Program Logic Manual describes about 20 private tables. It seems that most of this mass of information is $\mathbf{1 0 0}$ duplicated or derivable from some other table and efficiency considerations which provided the rationale probably now no longer apply. It is, however, impossible to restructure the data because the design group has long since lost control to various implementation groups who have been building local performance improvements and perhaps inadvertently taken over the function of the central design team. In other words nobody understands the system well enough to make any major changes. In any case the programs have been written so that only trivial changes in tables can be made without bringing on a rewrite of all existing code.

In summary, given a long fluid design, implementation and maintenance schedule, there will inevitably be a series of decisions made to improve performance. Each such decision will be based on current understanding which inevitably includes the fact that a major modification of logistics is not possible. Thus there is a tendency to invent new data structures which increase the complexity and reduce the level of overall performance. As the logistics become more complex there is a concomitant loss of design control which results in a further increase in complexity and decrease in performance. The evidence of this process is a sort of stratification of levels of information, observable in any large system, which is like an archaeological record of many attempts to improve performance.

The above requires some qualification. The confusion, which is observable in so many large systems, is also due to a great deal of "fence building" by those designing and implementing components. Given a task to accomplish and little understanding of the entire system, there is a tendency to define an environment which insulates oneself from the design perturbations of the entire system. While the motives are somewhat different, the effect of such local design also reduces performance. It is also apparent that "performance" must be described rather more broadly to indicate how serious are the problems of many current programming systems. Reliability, serviceability, and graceful degradation are often cited as goals. Complexity, which destroys the throughput of many systems, also makes the above goals unattainable. There is just too much information for the necessary simplicity and stability.

Ultimately the way out of this must be a new methodology for writing large systems. This would include the following:

1 An algorithmic programming language, which is free of most considerations of efficiency.

2 A compiler and run time environment which permit the development and check out of a large system without reference to its performance.

3 The process of making a choice of options and specialization for a particular hardware configuration at a particular location, commonly called system generation, which might also be the opportunity to bind design decisions that relate to performance.

Developing a system with such tools would permit several iterations of design and implementation to develop the required functional capability and to ensure accuracy. The system would then be bound for performance. Super optimizing compilers would treat all the modules in an optimal manner. Tables, data structures, and access methods would be restructured through a terminal system which would provide for co-operative optimization by a designer and system simulator-optimizer program. This in turn requires statistics supplied by accounting routines which function while the system runs in unoptimized form.

The technical problem which must be solved centers on the problem of binding. Loose binding makes it easier to change decisions but often results in a loss of performance. (In some sense optimization may be viewed as the 101 process of making binding decisions at the earliest possible time.) In our work at the T. J. Watson Research Center, the problem of binding storage has been one touchstone for discussing the performance problem. The conventional approach in FORTRAN, COBOL and assembly language is that while some or even most information about data is kept in symbolic format, there are some cases where structure is bound into the program at an early date. For example, PL/1 has CELL, FORTRAN programs have EQUIVALENCE statements which determine storage allocation in detail 
and COBOL programs treat a structure as an atom which can be manipulated as if it were an entity rather than a collection of entities. In these and other cases it is impossible to change data formats without changing, or at least examining, much of the source program.

An alternative approach is to treat data as objects in the manner of ALGOL 60. This is most attractive, especially if the historical situation of millions of lines of working code in other languages is ignored. Even ALGOL may not be ideal owing to its lack of data structures of greater complexity than an array. Unfortunately the languages which possess this attribute, such as LISP or M6, acquire it through list structure which seems to imply a high degree of interpretation or late binding which is very inefficient. The study of how to bind such functional languages at the earliest moment will yield very significant results, for it will then be possible to design and program in the same language. The basic techniques that will be employed in this situation are those developed for optimizing more conventional languages, such as FORTRAN, as well as new techniques for optimizing data formats and more global units, such as entire procedures. It is interesting to note that the emphasis will be different from that of FORTRAN optimizing. There will be less attention to operations that result in improvements in the 10 per cent range and more to those that yield orders of magnitude improvement. This is only possible in languages which have a very loose structure and are thus usually interpreted. It is just these languages that do not bind the data structure into the solution that are ideal for the designer.

Thus the optimizing compiler can be seen as the vehicle which permits software designers to work at a high enough level and still retain control of system design decisions. This should result in systems which are simpler, more reliable and perform better.

\subsection{Languages for writing system programs}

by

Charles A. Lang

I find it alarming that in spite of the enormous amount of research and development effort that has gone into programming languages by way of syntax definitions, theories of type, compilers, compiler-compilers and the like, the majority of system programs are still written in assembly language. Why is this? Is it desirable? If not, can anything be done to improve the situation?I shall not attempt to define "system program", but merely point out that I consider compilers, operating systems, program overlay schemes, storage allocation packages, on-line commands for a multi-access system and graphical input/output routines to be examples of system programs.

\section{2}

\section{Why assembly language?}

There are a number of reasons why assembly language is used.

1 Assembly language has no peers for producing efficient programs, that is, programs efficient in terms of compiled program size and speed of running. Efficiency does matter. People often pay lip service to it and then write extremely inefficient programs. High efficiency reduces operating costs and makes things possible that are impossible if the programs are inefficient. Two examples are fast console response to filing operations in a multi-access system and wringing the most out of a small (say 8K) machine. The skill and ingenuity of the programmer also have a great effect on efficiency, but this is just as true for high level languages as for assembly code.

2 Assembly language enables you to get as close to the machine as is possible. It does not prevent any operation that the machine is capable of executing from being programmed. This is certainly not true of high level languages. Further, the discipline of learning assembly language ensures that the programmer learns about the machine. High level languages screen the programmer from the machine. While this attribute is highly desirable in many applications, system programs can benefit from the programmer thoroughly understanding the actual machine and its capabilities.

3 Assembly language can be quickly compiled; this is a great convenience when developing programs, especially on-line with a single user or multiaccess machine.

\section{NATO SOFTWARE ENGINEERING CONFERENCE 1969}


4 On-line debugging systems are an enormous aid to debugging at the assembly language level. Typical facilities are interrogation and setting of registers, searching for bit patterns and break points, free running and interpretive execution and so on. Correction of errors with high level languages normally involves editing the source document, re-compiling and re-loading.

5 Assembly language systems do not require the use of a run-time system to perform functions such as space allocation. This leaves the system programmer with maximum flexibility to organize his system program to suit the problem at hand.

\section{Is the programming of system programs in assembly language desirable?}

The answer is no, but unfortunately it is still the most suitable language on many machines. Briefly, because they are well known, the very grave disadvantages of assembly language are:

1 Apart from the few who delight in such intricacies, most people find assembly language programs harder to write, read, understand, debug and maintain than high level language programs.

2 It provides the poorest conceptual framework for the programmer to express the computing operations he wants performed.

3 It is completely machine dependent, thus requiring any machine language program to be completely rewritten when it is transferred to a different machine.

\section{Can anything be done to improve the situation?}

The answer to this question is yes. A completely machine independent language particularly suited for writing system programs and which compiles code of comparable efficiency to that of a good assembly code programmer on every machine on which it is implemented, would be ideal. This is impossible with the present state of the art, so what sort of a language is it possible to produce now? I shall not define an actual language 103 but rather discuss what type of a language it should be. BCPL, MOL940, PL360 and SAL (see References 1, 2, 3 and 4 respectively) come a good way towards meeting the requirements, while EPL (see Reference 5) falls shorter. BCPL offers machine independence but falls short in other requirements discussed below compared to MOL940, PL360 and SAL.

\section{Clarity}

A programming language provides the conceptual framework within which a programmer must think about his problem, so influencing his method of solution. The language should enable the programmer to state clearly the computing operations he wants performed; further, the program must be clear to read both by himself and others. A high level language such as FORTRAN, ALGOL or AED meets these requirements better than assembly language, but these languages fail to meet the other requirements discussed below. The language itself must be easy to learn and have a short, well written programming manual with an index.

\section{Efficiency}

The language should be capable of being compiled efficiently to produce efficient code, say not more than 20 per cent longer or slower than the code produced by a good assembly language programmer. This is a great blow for machine independence. For if the language were to contain all the features desirable for system programs then it would not be possible to compile all the features efficiently on each machine because of the hardware differences (e.g. character operations implemented on one machine may be completely absent on another). Programmers could still write efficient programs for particular machines by using only those features of the language that they knew to compile efficiently on their machine. They would then, however, have the advantage of only a subset of the language. When programs were transferred from machine to machine the running efficiency would vary greatly.

\section{Simple and flexible language design}

The language should be simple, but not at the expense of its generality or practicability. Simplicity also aids efficient compilation to produce efficient code. The target should be a high power-to-complexity ratio; if the basic operations provided by the language are well chosen, then these provide the tools with which the programmer may fashion more complicated and specialized operations. Much can be achieved with a very general syntax, avoiding exceptions and

\section{NATO SOFTWARE ENGINEERING CONFERENCE 1969}


special cases as far as possible. BCPL is a particularly good example of this. Further, as much as possible should be left out of the language rather than the reverse. Stacks are often used in system programs so stacking operations might be included. To provide all possible stacking operations (first-in first-out, pushing and popping different numbers of words, popping the top item, copying the top item, etc.) is too complicated; if only a subset is implemented it may not include the operations programmers want. Far better to omit them altogether, since the language should contain basic operations from which stacking operations may be readily implemented.

The language must not prevent the programming of anything that the hardware can execute. Languages offer two types of restrictions here. First, the language may not have a construction, perhaps for a particular type of shifting operation or character manipulation, that is implemented directly in the hardware. When these operations are required, either the programmer must program around them, which is inefficient, or in most languages he 1-4 may call an assembly language subroutine. This too can be very inefficient when the number of orders executed to get into and out of a subroutine is comparable to or greater than the number in the subroutine itself. Secondly, the language may prevent certain operations that are highly useful. For example, the description of an "object" in memory may contain several items of data which it is convenient to store in a contiguous block. This data may be of mixed type, in which case FORTRAN would not permit them to be stored in a single array. The problem could be fudged around with an assembly language program but only very inefficiently and inconveniently. This is but one example of the restrictions that types impose. Types are a menace to the system programmer as they reduce the flexibility of a programming language enormously. They do enable the compiler (at some expense) to report on type mismatches. However, this is no staggering advantage since such faults are usually easy to find, especially if the programmer adopts some convention in his mnemonics to identify types which are conceptually different to him. Even using a language that has types, the programmer may still have to group under one type, say pointer, types that for him are different, such as tables, lists and character strings. Types also allow mixed mode expressions, which are marginally useful, and the repeated use of the same symbol to indicate different operations; for example, $+,-, *, /$ can be used for integer or real arithmetic. Without types different operators could be used, e.g. +, - , *, / for integer arithmetic and $£+, £-, £^{*}, \mathfrak{£} /$ for real arithmetic.

\section{Machine dependence}

Machine independent programming is to be applauded, but it must be recognized that there are times, even if very few, when machine dependent programming is desirable. For example, when very high efficiency is essential or intimate control is required over the machine, such as in the control of peripherals or loading of machine registers. Many operations in the language can be machine independent. Nested arithmetic and logical expressions, IF, FOR, WHILE, UNTIL, UNLESS statements, assignment statements and GO TO statements are examples. One way to provide machine dependence is to make assembly language statements valid statements of the language itself and able to refer to the same variables as in the high level statements. Special statements could be added to the language to make the frequently used assembly language statements somewhat easier to use, but this could also be achieved by the use of a macro generator. Advantages of the assembly language approach are that all possible assembly instructions are included, it is very easy to recognize the machine dependent parts on a print-out (important when transferring to a different machine), and it provides the most machine independent way of compiling a machine dependent language! If the compiler produces assembly language which is then passed through a standard assembler on a machine, then the compiler need only copy the assembly language statements while compiling the high level statements into assembly language. Conceivably a language could be designed with a machine independent part plus assembly language. A machine independent compiler could be written that would have to have its code generator changed when transferring from machine to machine (like any other compiler) but in addition would only have to be able to recognize the machine language statements of the particular machine so as to copy them, since they would require no further processing by the compiler. Users wanting to transfer programs from machine to machine could write solely in the machine independent part of the language, at the cost of efficiency, if they wished to minimize reprogramming.

\section{5}

It is highly convenient to be able to refer directly to machine registers in expressions as in SAL and PL360. This would give more, but not insurmountable, trouble when transferring the compiler, or a program from machine to machine.

\section{No run-time system}

The language must have no run-time system of any kind. That is, nothing should be loaded along with the program "behind the user's back", such as an input/output package, or run-time routines to operate stacks or dynamic use of

\section{NATO SOFTWARE ENGINEERING CONFERENCE 1969}


working space. In this sense it is like a conventional assembly language. This requirement allows it to be used to write any type of system, leaving the programmer quite free to control everything loaded into the core along with his system program.

\section{The consequence of a statement}

The consequence of each statement should be clear to the programmer. Hidden mechanisms should not be brought into play without his knowledge. Apparently there are statements in EPL which look simple and inoffensive on paper (e.g. for character manipulation) which compile to lengthy code and run incredibly slowly. It is advantageous to have the language simple enough so that programmers may, if they wish, even be aware of the code that the statement compiles into.

Ability to run together programs written in different languages

Despite many attempts, no programming language has yet been devised which is suitable for all programming tasks. Apart from this, the more comprehensive a language becomes the more unwieldly the compiler and system that go along with it tend to become also. Different languages may be most suitable for different parts of a single system. FORTRAN is unsuitable for writing a graphics package but a FORTRAN user may want to use such a package written in another language. Programs must, therefore, run within some mixed language system where programs written in separate languages may be compiled separately but loaded and run together. Many such systems exist but often the methods used in interlanguage communication, which are a function of the loading system as well as the languages, are limited to the FORTRAN requirement. This provides one way of calling procedures and passing arguments, plus communication via COMMON. As a result of these restrictions, some languages, notably ALGOL, are not included in a mixed language system. The same loader, however, is sometimes used to load into core the compiled code of these languages and of languages within such a mixed language system. A system language program must have the same freedom as an assembly language program to communicate with programs written in any language that may be loaded into core with it by the same loader. Desirable facilities are: COMMON, global variables (association by name rather than order as in COMMON), multiple entry points to separately compiled "hunks" of program and the ability to call procedures and pick up arguments from procedure calls, both written in any language within the mixed language system.

\section{Input/output}

Input/output operations are most easily provided by sub-routines.

\section{Summary}

In summary, the language should be simple enough to permit efficient compilation of efficient code. It must provide the programmer with a 106 comprehensive set of constructions to perform the most frequently required computing operations. This should be as machine independent as possible, chosen so that it can be compiled efficiently on the majority of machines. Any construction that, due to particular machine hardware, cannot be compiled efficiently should be brought to the programmer's attention in he documentation for that implementation. Further, the programmer should be able to include assembly language orders as a natural part of the language and be able to communicate with program statements in other languages. Based on existing experience, an estimate of the effort required to implement a system writing language on any machine is of the order of one first class man for one year (SAL took a manyear, PL360 rather less I believe).

\section{References}

1. Richards, M. BCPL: A tool for compiler writing and system programming, Proc. SJCC, AFIPS, 1969, p. 557.

2. Hay, R. and Rulifson, J. N. MOL940: Preliminary specification for an ALGOL like machine oriented language for the SDS940, Interim Tech. Report 2, Project 5890, Stanford Research Institute, March 1968.

3. Wirth, N. PL/360 A programming language for the 360 computer, JACM 15, No. 1. January 1968.

4. Lang, C. A. SAL—Systems assembly language, Proc. SJCC, AFIPS, 1969, p. 543.

5. Corbato, F. J. PL/1 as a tool for system programming, Datamation 15, No. 5, May 1969.

\section{NATO SOFTWARE ENGINEERING CONFERENCE 1969}




\title{
7.9 UNIVAC 1108 instrumentation
}

\author{
by \\ J. M. MacGowan, Jr.
}

\section{Introduction}

For a number of years the 1108 project has been interested in the development of techniques to observe closely the operation of 1108 processing systems. Insufficient information is available on precisely how an 1108 is used. The frequency of instruction usage, the sequence of events in operation and the balance between input/output and processing are all areas of interest in examining current system performance. That the 1108 can provide correct answers is insufficient. We are vitally concerned with the manner in which it goes about developing these answers.

Simulation is an important tool in the implementation of complex systems. But the observation and measurement of system performance is equally valuable. Techniques of evaluation for finding system deficiencies are as important as the tools used to construct the system. The interaction of thousands of decisions in a product as complex as the 1108 operating system are extremely difficult to simulate accurately and simply. It is equally difficult to visualize the net result of operation of many small components, even though the logic of each component is well understood.

We are aware of many prior attempts by others to instrument their systems in order to gain a more thorough understanding of their operation. 107 Analog devices have been used to meter the use of various system components. The rough percentage of tape channel utilization would be an example of the output derived. Others, with special hardware devices, have recorded partial samples of the instruction execution sequence. One device wrote the data directly on to magnetic tape. The relative speed of the tape was so slow however that only a small fraction of the data points could be recorded. A number of papers have appeared lately which use a real time clock interrupt to interrupt the instruction execution sequence frequently and $\log$ the point at which execution was occurring. Again, only a statistical sampling of what that machine is doing is possible. Particularly, software instrumentations contribute to system overhead in almost direct proportion to the quantity of data captured. Any significant interference with the process observed can distort this process so severely as to make the data captured unusable. We have experimented with the insertion of counters in our Executive to record the frequency of key events. This recording is very limited and does not relate the time sequence in which the events occurred. To limit the overhead of the software instrumentation a minimum number of counting sequences can be inserted. You must predict the desired information, insert the counter by hand and go through an iterative process until the proper data has been recorded. We once instrumented the routine which controls all data space allocation in the Executive in order to develop a better understanding of the requests and releases of buffer space. To protect the timing relationships within the system, one entire tape channel was dedicated to recording the output. While we were successful in recording the sequence of buffer requests and releases, the information gathered presented no relationship with the other components of the system. The tape channel was able to record only this one small set of events.

\section{Instrumentation system description}

The instrumentation approach described in this paper makes use of data collection hardware and software to monitor activity in one processor for presentation to another processor for recording. Neither the insertion of

monitoring hardware nor the operation of the collection software in any way affect the operation of the monitored system. Therefore, data received is an exact record of system operation. An absolute requirement for the instrument was that there be no impact whatsoever on the system being observed. The operating system must be unaware that it is being watched.

The system consists of three basic parts:

1 A hardware monitor to gather data from a monitored processor and to present that data to an I/O channel of the recording processor. The data gathered consists of absolute addresses to which jumps are made, time base information, information on the state of the monitored processor, and loop summaries.

2 Data collection software used by the recording processor to gather data from the monitor, to temporarily store that data on drum, and to prepare tape output for data reduction.

3 Data reduction software to analyze the collected data.

Monitor logic

The monitor logic consists of five basic sections: 
1 Logic to detect the execution of jump instructions within the monitored processor and to capture P-register values relating to those jumps.

108

2 Registers to provide a buffered data path from the monitored processor to the recording processor.

3 A free running time base with a $0.5 \mu$ sec period to provide time information relating to jump occurrences.

$4 \quad$ Jump loop detecting and counting logic.

5 Control logic to sequence data flow through the monitor.

\section{Monitor hardware}

The monitor device is built of a logic family compatible with the monitored processor and is mounted on a card rack inside the monitored processor. Power and clock are obtained from the monitored processor. Connection to the recording processor is via the input cable of a standard I/O interface. The figure shows the monitor installed in a partitioned multiprocessor system. This hardware is applicable to other processors using the same logic family after appropriate changes in the monitor logic, to detect jump executions and sampling of the P-register and processor state information, have been made.

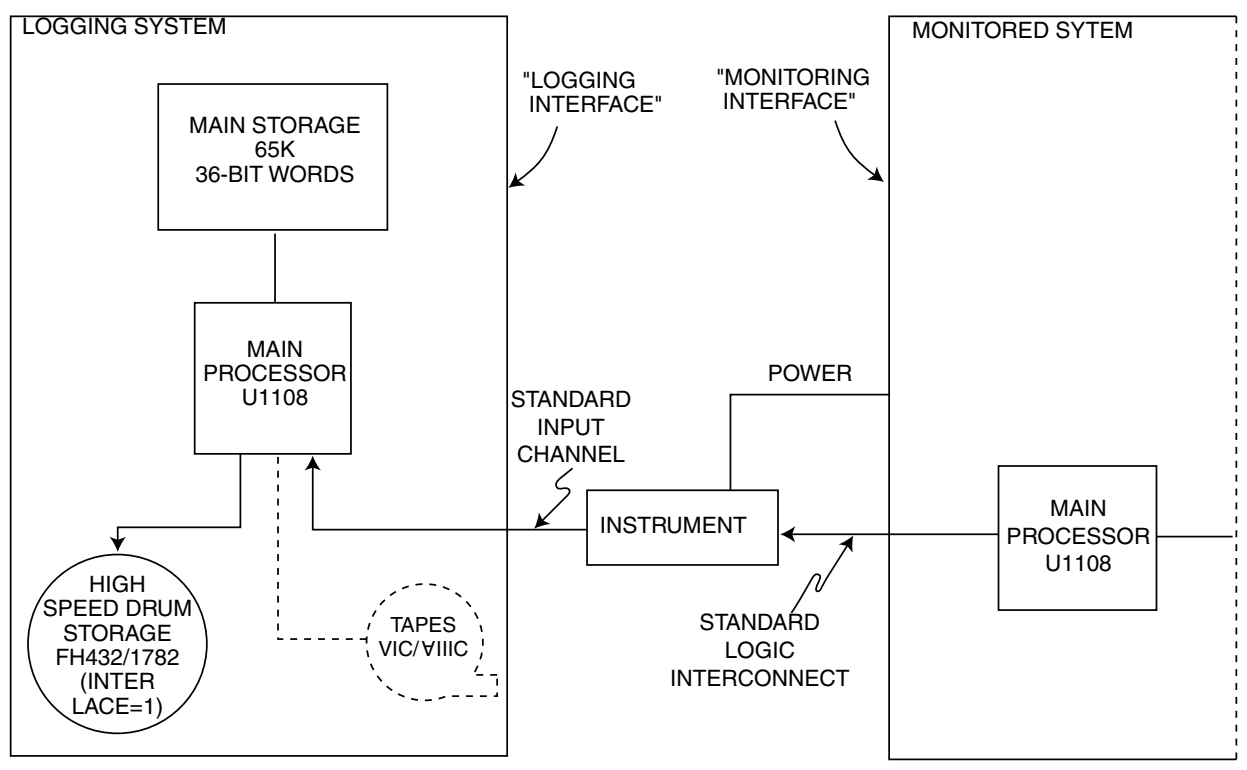

Figure 1. Instrumented system configuration, functional block diagram

\section{Monitor operation}

The monitor creates a data word each time a jump instruction is executed in the monitored processor and when certain time and jump loop conditions occur. At the time of jump execution the absolute value of the jump address is extracted from the processor and inserted into the first of a series of buffer registers in the monitor; the time and jump loop conditions are described below. The various fields of the data word are as follows:

S (bits 0-17): Contains an 18-bit absolute P-value of the address to which a jump is made, or a 9-bit value in bits 0-8 defining the number of times a single loop was executed, where a simple loop is defined to be two successive jumps to the same absolute P-value.

$\mathrm{T}$ (bits 18-26): A time value defining a relative time at which the data word was created. This time is derived from a free running time base in the monitor which is incremented by one every $0.5 \mu \mathrm{sec}$. A maximum time of $256 \mu \mathrm{sec}$ can be defined by the $\mathrm{T}$ field. 


\section{9}

F (bits 27-29): Bit 27 defines the content of the S field: Bit 27=0 defines $\mathrm{S}$ to be an 18-bit absolute $\mathrm{P}$-value, bit $27=1$ defines $\mathrm{S}$ to be a 9-bit loop count. A data word having bit $27=1$ is defined as a loop summary word. Bit $28=1$ defines a timer overflow condition, which marks a $256 \mu \mathrm{sec}$ time interval. Bit $29=1$ defines a buffer overflow which implies that the monitored processor and the monitor are generating data words at a rate faster than the recording processor can accept words; a data word with bit 29=1 represents invalid data.

M (bits 32-35): These represent the state of various bits of the processor state register (in the monitored processor) at the time of a jump execution.

\section{Loop summarization}

Since the data rate produced by jumps in "typical" coding is of the order of a jump every $5 \mu$ sec the monitor contains hardware to perform limited "on the fly" data reduction. This reduction is done by detection of simple loops (i.e., two successive equal P-values). Only two data words are sent to the recording processor to describe a loop. The first word defines the first loop jump; the second word defines the number of times through the loop and the time of the second loop jump. Therefore, the length of loop and the total time in a loop can be calculated.

\section{Data collection}

Collection of data words from the monitor is done on a system which is independent of the monitored system. This recording system may be a physically separate system or a portion of a partitioned multiprocessor system. It is necessary that the core storage and drums used are private to the recording processor so that the recording activity does not affect operation of the monitored system. Collected data is transferred to drum via two large core storage buffer areas in the recording processor. The length of monitoring period is a function of the number of drums available to the recording system. The collection software allows selection of recycling a drum area or transferring from drum to tape at the completion of filling a drum. The recycling mode permits maintaining a recent history prior to some stop point.

\section{Data reduction}

The purpose of the instrument discussed in the first part of this paper is to approach the problem of inefficiencies in a complex data processing system by concentrating on a narrow slice of the problem; namely, inefficiencies in the structure and utilization of software, and in particular, the Operating System. Owing to the nature of the data collected (i.e., this instrument captures a dynamic picture of a non-degraded Operating System), the information which is obtained on the monitored system through data reduction will provide a unique perspective on software performance. In particular, both statistical and graphical results will provide facts whereby software performance can be evaluated by not only whether certain specified functions are performed, but also how efficiently they are performed. The initial results of data reduction seem to indicate that a step in the right direction has been taken in the development of a capability for identifying and eliminating software inefficiencies.

As dictated by input, a structure is placed on the data in order to organize it into a form more amenable to analysis. This structure consists of matrices of data called sub-divisions, which are defined to be restricted time and/or P-ranges. Generally, only the P-ranges will be restricted and these will be coincidental with known logical sections (e.g., the resident elements $\mathbf{1 1 0}$ of the Executive system). The usefulness of this structure will become apparent when discussing the statistical and graphic-associated printout.

\section{Graphic results}

The graphical output simply consists of a plot of P-vs. time-values over any portion of the P and time-ranges monitored. The user is able to obtain both gross and detailed graphs by varying the number of microseconds plotted per inch. The gross plot is adequate for the user familiar with the detail of various activities as these will leave a "signature" through the graphical pattern displayed on the chart. Those areas with which the user is not familiar can be expanded, i.e. a detailed graph with fewer than $\mu$ sec per inch for the area of interest. Once the user is able to correlate a particular graphical pattern with an activity, there is no further need to examine that area in detail. Also, there is the capability of obtaining a detailed columnar print report which gives the time, the subdivision into which the jump occurred and the relative location of the jump within the subdivision. This print report is then used together with the graph and a listing of the monitored program in order to determine the activities performed. It has been found that

\section{NATO SOFTWARE ENGINEERING CONFERENCE 1969}


graphical patterns are easily correlated with various activities, thus giving a strikingly clear picture of the sequence of events.

This dynamic picture of the monitored system is not only useful to programmers knowledgeable in the activities being monitored, but also has been found to be a valuable training aid for those unfamiliar with the operating system. Flowcharts, manuals, program listings, etc., are static aids which only tend to come alive when verbal descriptions of various activities can be visualized through the graph and by following the dynamic flow of the logic using the detailed print.

Also, it should be noted that this graph and its associated print can be a useful debugging tool. Should a particularly difficult bug exist in the system, then the recording of environmental data just prior to the detection of a system or program failure will provide a high speed trace capability since the instrument will capture an accurate record of the system destroying itself.

\section{Statistical results}

The data reduction program also provides the capability of obtaining statistical information on the subdivisions; this consists of such items as percentage of time spent in each subdivision, percentage of time spent in the Executive system mode, number of times the subdivision is entered, etc.

Once system inefficiencies have been removed using information gained from the graphical method described above, then the user may collect statistical summaries for various simple job mixes. Then, when more complex job mixes are introduced, the system can again be instrumented in order to determine what new time distributions have resulted from the more complex mix. It may be found, for example, that use of certain executive elements is highly correlated to the new mix causing them to be executed a disproportionately large percentage of the time, and this would show up in the statistical results of an experiment on the instrumented system. The user would then determine whether inefficiencies had been introduced with the new job mix and, if they had, whether the inefficiencies stem from the method of using the equipment, from software inefficiencies not previously detected or, perhaps, from an inherent system inability to handle the particular mix.

MacGowan's paper continued with a detailed example of this method keyed to samples of extensive graph-plotter output. This is not reproduced here (Eds.)

111

\subsection{Software engineering techniques and operating system design and production by \\ R. M. Needham}

Operating system production perhaps presents rather a different challenge to the software engineer than do some other forms of the art. Two of the reasons for this are the liability of operating systems to contain unrepeatable faults and the fact that operating systems frequently have to be developed for computers which do not have a very large amount of support software in existence already. For reasons of this sort it is perhaps even more essential than elsewhere to proceed in an orderly and organized manner and to devote substantial amounts of effort to software of a diagnostic or other ancillary nature. This note mentions some points which have occurred to me on this topic, some of them being allusions to things that I feel should be provided or done and some of them mentioning difficulties and unexpected pitfalls.

It is quite essential to equip oneself with adequate mechanized means for handling the source text of one's system. If it is to be a system of any complexity, it will presumably be able to handle its own source text when complete. However it clearly cannot do so before it has been written. One is likely, therefore, to be involved either in using another computer for performing this file maintenance function or in writing a temporary system which will be thrown away when the main system is at a sufficiently advanced stage. However, there is a danger here. It is very awkward if the ways in which one handles the text in the earlier stages are grossly different from the ways in which one handles it later on. This can be extremely hard to avoid; if I am handling the text for a new system on an existing one, it is probably the case that the conventions for such operations on the new system will be different and there will have to be a possibly painful period of turnover and retraining at a nasty time in the middle of the operation. My feeling is that this problem has to be faced and circumvented as best one can.

\section{NATO SOFTWARE ENGINEERING CONFERENCE 1969}


It is commonplace to realize that earlier decisions in the designs of an operating system are of great importance, because they may very well determine the logical structure of much of what follows and the general shape of interfaces between different parts of the system. One therefore knows that it is necessary to get the early parts right, because if they are subsequently to be changed a very large amount of work may be involved. However, commitment to a particular structure can arise from a rather different source, which is not so obviously connected with the basic system. If one is to preserve sanity and to make any kind of regular progress, there is going to be a need for software aids to the diagnosis of faults in the system under construction. These take a considerable variety of forms; there are routines for permitting on-line inspection and changing of registers from, for example, the operator's console; there are programs that are interposed in the calling sequences of one routine to another to check that things are being done properly; and there are packages designed to facilitate the understanding of core-dumps. It may be worth elaborating on this last. If one is dealing with a flexible operating system for a large machine, it may easily be the case that a particular part of the core store may be used for a large variety of purposes, both system and private, at various times. If there is some obscure crash in the system it is not much good expecting to 112 take an octal dump of $1 / 2$ million bytes of core and leave one's programmers to thread their way around it. Most system problems leave the core in a fairly respectable state, and it is practicable and almost necessary to equip oneself with a package that knows about the data structure the system uses, knows the values of important parameters in it, and arranges to print out an interpreted map of the corestore when wanted. Now the point I am getting at is that a very considerable and thoroughly justified investment is frequently made in this kind of software, and it must not be overlooked that what may be comparatively minor changes to the structure of the system as a whole can lead to a radical upset in the diagnostic software. Since the function of this software is to be thrown away once it is finished with, one does not wish to become involved in rewriting more often than necessary.

Having alluded to a point about rewriting, it is perhaps not wholly out of place to add a comment on rewriting parts of the system itself. Inevitably this happens; sometimes because a piece of it was written incompetently, sometimes because the specification one expects of a piece has to be so radically revised as the result of experience that nothing short of a rewrite will do. It should be urged, however, that this sort of thing should not be planned for in advance. Experience strongly indicates that putting in pieces of temporary and ill-thought-out software, often written by junior and inexperienced people, in order to have something to plug a gap, is often disastrous. In our own experience whenever we have yielded to the temptation to do something deliberately temporary the results have been bad. Either it never gets replaced at all, or when it does get replaced all kinds of problems arise. A trivial example could be in the case of a routine for organising disc transfers. One may start with a version which does no optimization, and executes the requests in strict order of seniority. A later optimized version may perhaps deviate from strict order of seniority in order to achieve its optimization, and thus expose horrors when it is discovered that other pieces of the system rely on a piece of behaviour which no longer occurs. Notice that it is not in any direct sense a case of getting the interface wrong. The way in which one asked for a disc transfer to be done would be the same in each case, as would be the way one would be informed that it had been done.

Once one has a going system, it is almost certain to get used for holding useful information, even if we are talking about a machine which is dedicated to system development. This point applies with much greater force to special purpose operating systems which are designed to work on a single machine in a single place for a single purpose. It is essential in these circumstances, and very desirable in others, to be able to run one's system in some kind of testing mode in which it is insulated as far as possible from harming valuable information. It can, for example, have special disc areas reserved for the purpose and, when it is loaded, have diagnostic facilities in it that would be uneconomic in a running system. It is important that the switch between a testing version and a running version should be easy, straightforward and automatic, so as to minimize the occasions when something works under testing conditions and not under real ones. Allied to the problem of testing versions is the problem of fallback. It is bound to happen that while the system is being run with a particular version of the system operating in it something will happen that is clearly a bug. It must be possible to fall back to an earlier version with as little fuss and confusion as is achievable or there will be a temptation to rectify the existing version by patching the binary. One of the most important software engineering techniques for operating systems is a negative one: do not patch the binary. In order to achieve this kind of fullback it is necessary to plan at a very $\mathbf{1 1 3}$ earlier stage what one's warm-start mechanism is going to be and to arrange, wherever humanly possible, that successive versions of the system are warm-start compatible. It is an obvious consequence of this that any changes that are made that affect, for example, the format of the data upon which warm-starts are based, have to be planned and executed with extreme care. If this has been done, it is then possible to arrange that, if a system crash occurs, in almost all cases the computer operators can cause diagnostic information to be 
recorded and fallback to an earlier version in a smooth manner taking, shall we say, less than two minutes without the intervention of any system programmers. The very possibility of doing this makes it much easier to introduce minor modifications.

Early in this note I talked about the necessity of having satisfactory ways for handling the source text of one's operating system. One of the important things about this is to recognize that, very commonly, the same text will be worked on by several people and to arrange one's handling system accordingly. No matter how much one thinks that one's system is divided into pieces, and each piece is only touched by its master, this is not the way things happen. The best technique is certainly one based on storing edits separately from the original document, only making a new version of the original document at fairly rare intervals. It is probably worth going so far as to arrange that whenever a new edit is inserted in the edit document the system automatically appends to it the date, time and name of person involved. If one's system is not capable of doing that automatically, then it should be.

Of these points some, perhaps all, are obvious. It has to be emphasized that they add up to a very considerable amount of planning work and coding work. This work should be done by one's best people, not one's worst people. It is accordingly expensive. It is however about as appropriate for the software engineer to try to by-pass it as it is for the civil engineer to try to avoid expensive heavy earth-moving equipment, relying instead upon armies of men with baskets.

\title{
7.11 Software engineering and computer science
}

by

\author{
R. M. Needham and J. D. Aron
}

Computer science aims at defining general principles underlying the design and application of software systems. It regards elegance and consistency highly, and tends to ignore small and awkward corners in its subject matter. Thus the computer scientist is tempted to analyze and treat that fraction of his problem that is amenable-rather in the same way that the differential equation expert treats the vanishing subset of equations that are analytically soluble.

The software engineer wants to make something which works; where working includes satisfying commitments of function, cost, delivery and robustness. Elegance and consistency come a bad second. It must be easy to change the system in ways that are not predictable or even reasonable-e.g. in response to management directives. At present theorists cannot keep up with this kind of thing, any more than they can with the sheer size and complexity of large software systems.

A theory must be independent of implementation. In practice large systems implementation is influenced by many factors: available personnel, $\mathbf{1 1 4}$ management structure, and so on. If theoretical work cannot adapt to this it can at best do no more than give helpful hints, while at worst it is irrelevant or unpractical.

Much theoretical work appears to be invalid because it ignores parameters that exist in practice. Thus a system that depends on failure-free operation is unrealistic. In the sense that medical diagnosis is empirical rather than scientificbecause the observable parameters are an insufficient subset of the operable parameters-so will computer science be empirical until better methods are available for describing the structure and behaviour of actual systems.

Theoretical work becomes impractical when it ascribes (mathematically) analytical behaviour to software —or at least to the software design, development and test processes. Present day software is the sum of individual activities operating in discrete and usually asynchronous time steps with a presumed common goal. The time steps depend on such factors as individual productivity, computer availability, predecessor activities, conflicting demands or resources, and quality of task specification. The common goal is "presumed" because semantics cause individuals to interpret the goal and its constituent tasks according to their own backgrounds. To contribute further to the random discrete behaviour of the implementation process (looked on as a system) is the necessary iterative nature of the goal itself.

Software engineering managers learn how to make decisions under conditions of uncertainty. Where possible, they reduce the uncertainty by applying theory or standards. In no case do they assume that a course of action will go perfectly and not require redirection. Theorists have not learned to cope with this randomly discrete set of events in an uncertain environment. Therefore, their impact on the engineers is minimal.

\section{NATO SOFTWARE ENGINEERING CONFERENCE 1969}


If theorists could demonstrate the application-independent value of their conclusions and could guarantee that they would work reliably on another job, engineers might overcome their conservative attitude toward new techniques and try them out. Unfortunately, an adequate demonstration requires two or more different large-scale implementations. This is usually beyond the scope of the theorists' interests or resources.

Pending major theoretical advances, software engineering should concentrate on the development of, and the exchange of experience about, practical tools such as:

diagnostic aids

protected testing facilities

automatic or semi-automatic fallback

aids to continuity during development, etc.

We believe that there is much to be gained by discussion and development in these areas.

\subsection{Compiler writing techniques and problems}

by

H. Schorr

It has been stated that Translator Writing Systems (TWS) represent the most serious attempt yet to automate software production. It might be better restated that TWS techniques have introduced formal or mathematical $\mathbf{1 1 4}$ techniques into one phase of the compiling process; it is not clear that they have really addressed the engineering aspects of that phase. This paper will try to fit TWS into the context of other methods for writing compilers, examine some of the shortcomings of each method and point out some of the areas that need research; examples will be taken from some industrial compilers.

\section{Categories of compiler engineering techniques}

Ad hoc techniques

Compilers written in machine language can be said to represent the use of ad hoc techniques. However, the understanding of the compiling process has reached the stage where the structures of most compilers are as shown in Figure 1; the standard phases being: statement recognition, storage allocation, optimization, code generation and output editing.

\section{6}

Statement recognition:

The first phase may consist of a prescan (to determine the statement type in FORTRAN, for example) and a parsing of the input string. A symbol table is developed during the parsing process along the way thereby reducing the size of the input string and determining the identifiers, constants, reserved words, etc. The output is usually in Polish form.

\section{Storage allocation:}

This phase allocates storage for variables, arrays, structures, format lists, parameter lists, etc.

Optimization and scheduling:

The basic machine independent optimizations most usually employed are the elimination of common sub expressions, removal of code from loops, reduction in strength (for example: conversion of array addressing to index register incrementation), etc. (see Reference 3). Scheduling refers to the machine dependent optimizations such as general and arithmetic register allocation and, in look-ahead type machines, the scheduling of machine operations to minimize the execution time of the statement or loop (see Reference 2). This phase is the one most commonly eliminated or combined within other phases.

Code generation:

This phase generates relocatable machine code from the internal form using the dictionary. Also, many object code optimizations that essentially are locally applicable coding tricks are carried out here, for example: replacing a multiply by a power of two by a shift. 


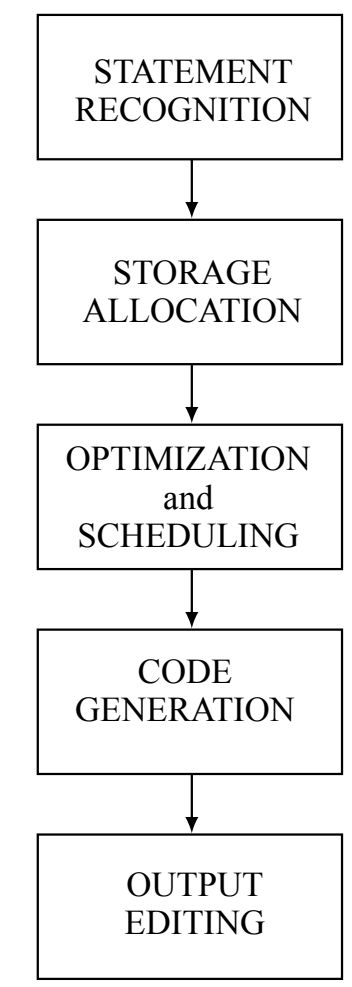

Figure 1. Phases of a compiler

\section{Output editing:}

This phase produces the actual decks and listing and may carry out the assembly of the code if this has not been done in the previous phase.

\section{Comments:}

The IBM FORTRAN G and FORTRAN H compilers fit pretty well into the above categorization. The FORTRAN E compiler, which operates in only $15 \mathrm{~K}$ bytes of core, has many more phases (12 plus 3 interludes) and is less coherently organized. The COBOL F compiler loosely fits the categorization for each COBOL language division but must also handle the interaction between the divisions.

\section{Table driven techniques}

Table driven techniques included in this broad category are all of the techniques reviewed by Feldman and Gries (see Reference 1). Broadly speaking, some formal description of the language to be compiled with perhaps the semantics of each production, usually in table form, serve as one input to the compiler. The compiler uses this input to parse the source statements. The main advantage of this approach is that the tables can be changed to those for another language; these tables are usually highly stylized and rewriting them requires much less work than the rewriting of an entire compiler. Such compilers therefore can be looked upon as the framework or scaffolding for the production of a processor for any particular language. Table driven compilers essentially use the tables in an interpretive way, i.e., scanning the source and matching it with productions in the tables. In industrial compilers these techniques have been employed mostly in the front end scan or parsing phases.

\section{Compiler writing machine}

This set of techniques consists of defining a set of macros or functions in which the compiler is to be written, instead of being written in instructions. 117 This presumably turns a general purpose computer into a computer which is better designed to write compilers for, i.e., a compiler writing machine. Included in the above are compiler writing 
computers whose machine language is one of the common higher level languages such as NELIAC, FORTRAN, etc. A compiler written in one of the latter languages is usually compiled so that the compiler writing oriented language is turned into true machine language. Various forms of this step are usually called bootstrapping. Examples include the NELIAC compilers and the IBM FORTRAN H compiler. In addition to the above a compiler writing language can be interpreted, two examples of this are the IBM FORTRAN G and FORTRAN D compilers. The interpretive method has the advantage of taking very little memory space and thus can be profitably employed when space is an important consideration or when an all in core compiler is desired. Some speed is lost but may be made up by not having the I/O delays while loading various phases. Transferability is enhanced because primarily only the interpreter (which is relatively small) has to be rewritten for a new machine.

\section{Large primitives}

Large primitives consisting of phases of an old compiler can be used to speed the production of a new compiler. That is, parts of an old compiler are borrowed, in toto, to make a new compiler. On an even richer primitive level an existing compiler can be re-interfaced with a new operating system. Within IBM both of these techniques have been employed and their use will probably increase as more and more types of compatibility are demanded by the users.

Another proposed method of reusing components is by having a well defined language (which may include symbol tables and pointers) for expressing the input to each of the well-defined phases of Figure 1. In this manner, for a given size compiler a new language can be added by changing only the syntax and probably storage allocation phases.

\section{Problems in compiler design}

The discussion on techniques of writing compilers has glossed over the difficult problems in writing a compiler. Some of the problems such as those encountered in the statement recognition phase have been subjected to considerable study and the application of mathematical or formal methods. It is this area that the bulk of the work of TWS is applicable, but unfortunately seems to account for between only 5 per cent and 10 per cent of the code; the original FORTRAN compiler using inefficient methods also did not exceed this bound. Other areas that might profitably be subjected to more formal study are optimization, error diagnostics, debugging, the nonregularity of languages, data structures, *code generation and register allocation. In addition to those features of compiler design that may be 118 amenable to more formal treatment there are many software engineering questions that arise for the compiler itself and the object code modules, i.e., at compile time and run time. These are mainly questions of resource allocation, interface with the operating system, maintenance and documentation and libraries.

\section{Resource allocation}

The compiler usually must be designed to utilize only a portion of the resources that are available in a system. A prime constraint is the amount of core in which the compiler itself must operate. This means that spill and overlay techniques must be developed. For example: IBM FORTRAN H while $403 \mathrm{~K}$ bytes long executes out of $89 \mathrm{~K}$ bytes; the overlay structure is shown in Figure 2. Choice of residence device and I/O techniques also radically affect performance. The I/O requirements for the FORTRAN H compiler aside from overlays are shown in Figure 3.

Within the compiler itself the choice of table structure and data representation radically affects the size of the compiler and the size of program that can be compiled. For example, in the IBM FORTRAN E compiler if the dictionary and the overflow table (which contains all dimension and subscript information) overlap (each one being built from one end of storage towards the middle) no new entries are made.

\section{Operating system interface}

Interfacing the compiler and the load modules produced by the compiler is a nontrivial task. Optimal use of the operating system and the translation of language features into standard operating system procedures is required. The IBM

\footnotetext{
*The effect of data structures on language processors can be dramatic. We quote from an unpublished memorandum by A. Opler. "The variability of permitted data types and data structures in a language has a dramatic effect on industrial compiler design and performance. FORTRAN I and II with only two data types and a simple data structure were fortuitous starting points in compiler development. As the complexity of data types and structures increase, the compiler complexity soars. The problems occur in (i) parsing and representing these data structures, (ii) generating and optimizing procedural code for their manipulation and (iii) more complex interaction with whatever part of the environment handles external data and backing storage. JOVIAL, COBOL and PL/1 are in a different ball-park' because of their data description capabilities."
} 


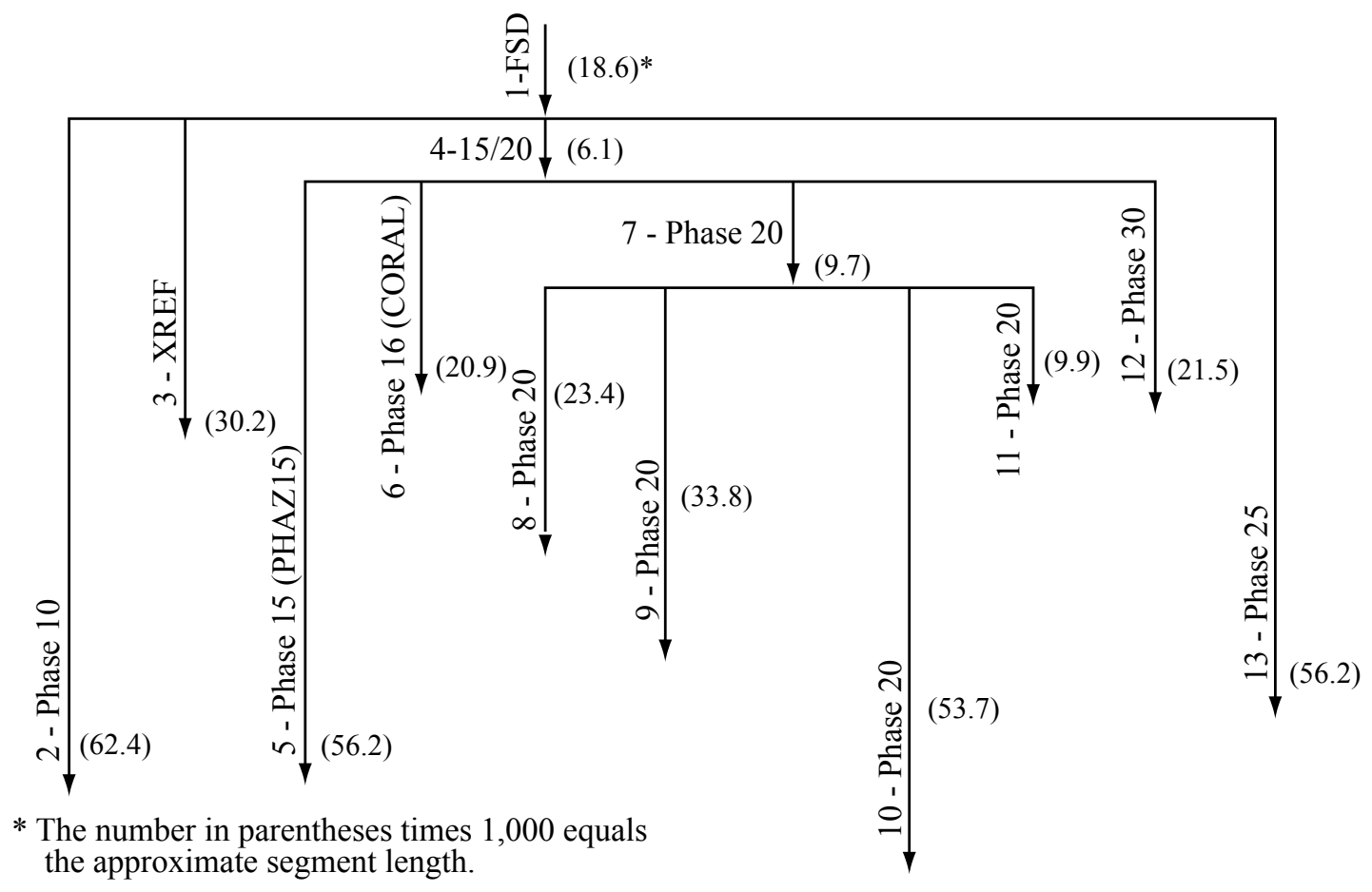

Figure 2. IBM FORTRAN H compiler. Overlay Structure

FORTRAN H compiler has an $18.6 \mathrm{~K}$ byte main segment which is the system director; it initializes the compiler, loads compiler phases, allocates storage, processes I/O requests from the phases, generates initialization code and deletes and terminates compilation. Similar phases exist in the other IBM compilers.

Similar interfacing exists at run time and is handled by library subroutines. An example for the IBM FORTRAN $\mathrm{H}$ compiler is shown in Figure 4 for an I/O request.

\section{Maintenance and documentation}

Care must be given to the design of the compiler to permit debugging and maintenance. This includes the design of good interfaces which minimize the amount of information to be passed but not necessarily cost. Several compilers include tools for debugging the compiler itself. Documentation is necessary to aid in maintenance since the latter may often equal the cost of developing the compiler in the first place. The diagnostic system into which the compiler is embedded plays a crucial role in debugging object modules.

\section{Subroutine libraries}

The design of a subroutine library is usually required for a commercial compiler. The IBM FORTRAN $H$ has the following object time library subprograms:

IHCFCOMH (object time I/O source statement processor)

IHCFIOSH (object time sequential access I/O data management interface).

IHCNAMEL (object time namelist routines).

IHCDIOSE (object time direct access I/O data management interface).

IHCIBERH (object time source statement error processor).

IHCFCVTH (object time conversion routine).

IHCTRCH (object time terminal error message and diagnostic traceback routine).

IHCFINTH (object time program interrupt processor).

IHCERRM (object time error message processor. The module monitors all execution time errors).

IHCADJST (object time boundary adjustment routine). 


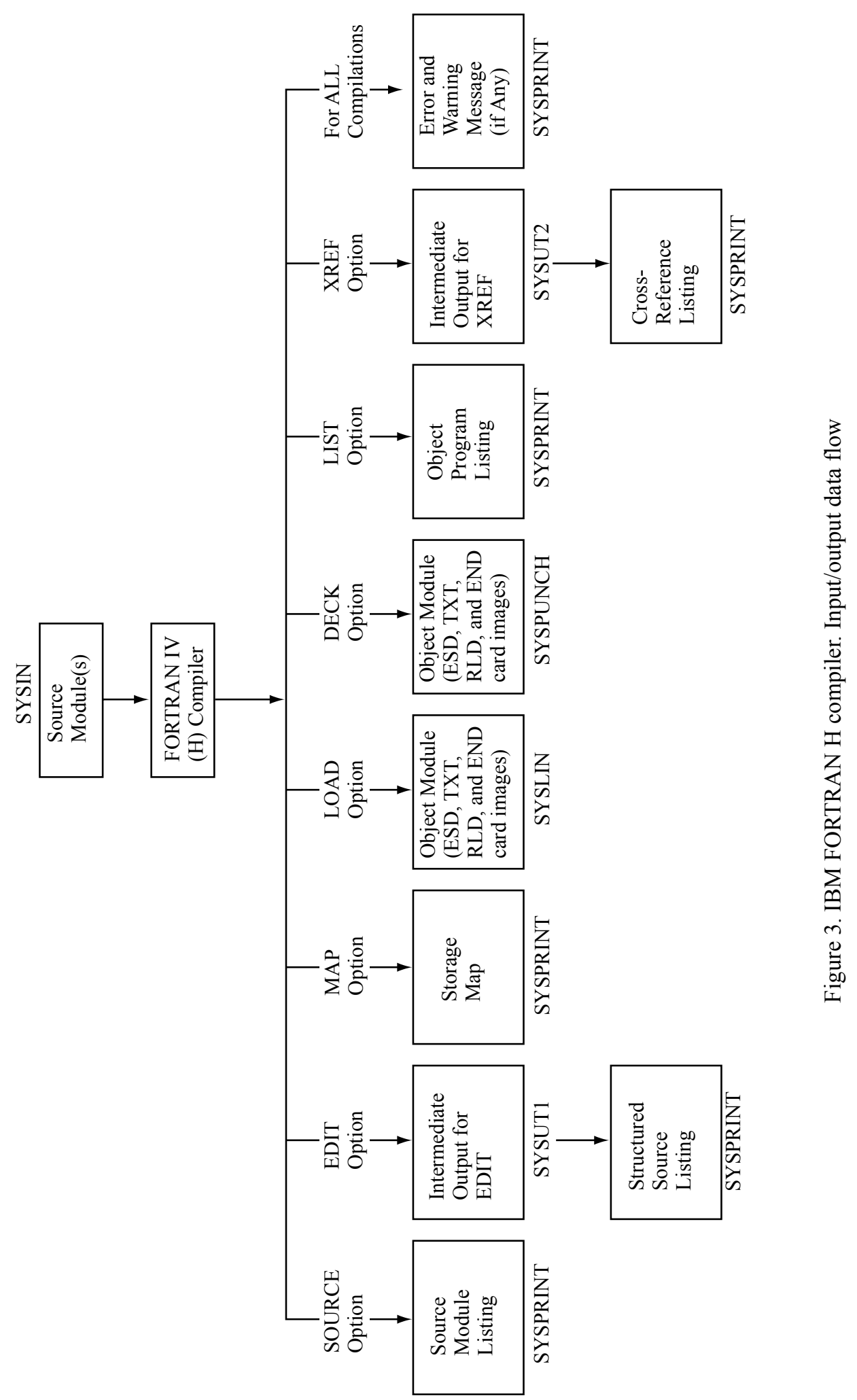




\section{1}

Most of these subroutines are also available in another version which provides an extended error message facility. In addition, a new function library both single and double precision, must be provided for a new machine.

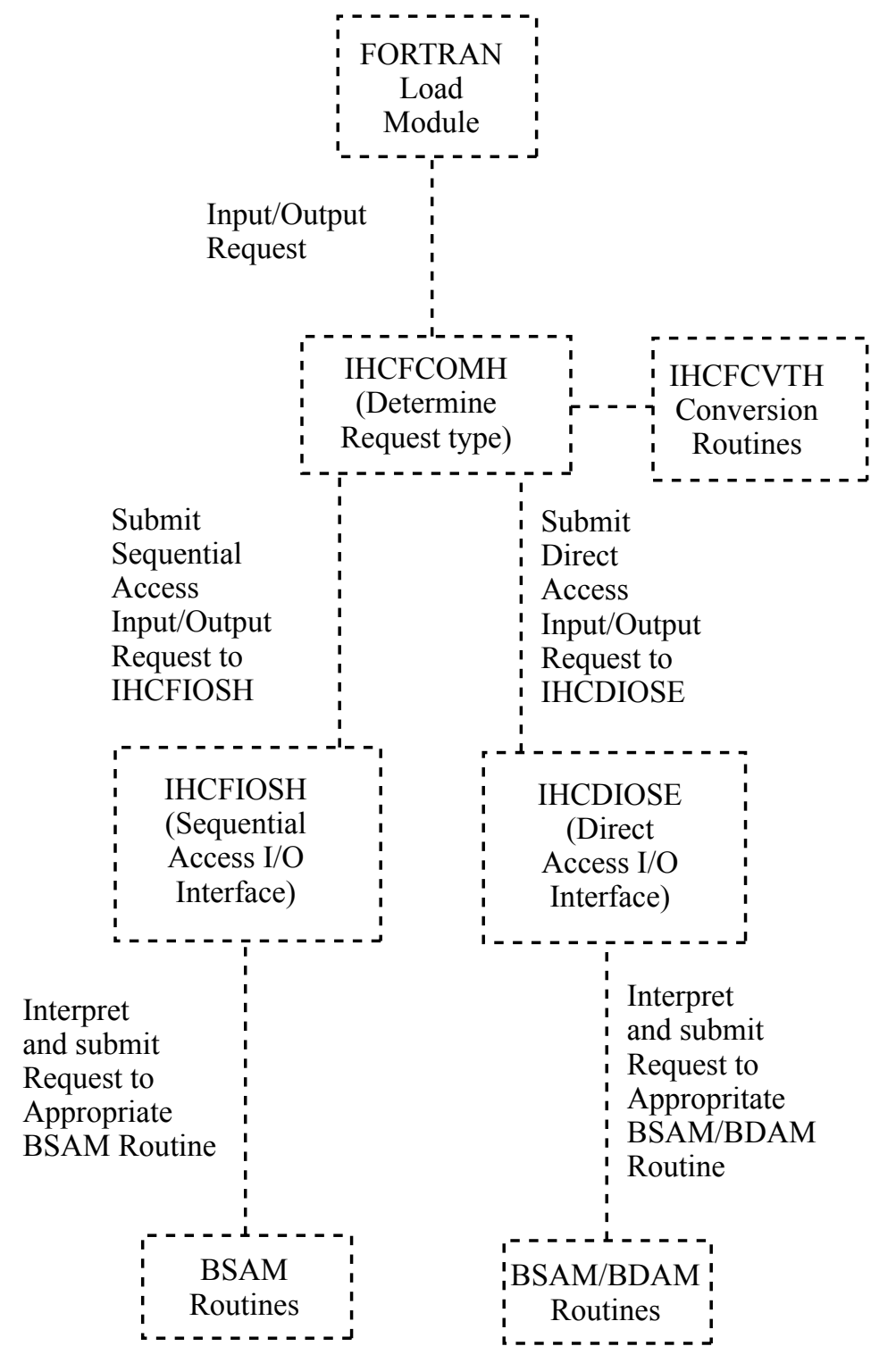

Figure 4. IBM FORTRAN H compiler. Object time library subroutines for input/output data management interfaces

\section{Shortcomings of techniques}

Ad hoc techniques

These techniques fail in their ability to accept language changes easily and in the lack of a set of consistent design principles. The cost and schedule delays result from this. 


\section{2}

Table driven techniques

Papers on these techniques, and many of the TWS compilers written neglect and do not clarify any of the engineering considerations; very little is said or done about the problems faced in the last four phases of the usual ad hoc compiler. A major difficulty usually encountered with these techniques is in producing good object code and error diagnostics. Compiler-compilers face the difficulty of interfacing with new operating systems when the compiler is produced for another machine. Space requirements may also often be larger than those of hand coded compilers.

\section{Compiler writing machines}

The interpretive techniques seem to run into difficulty in providing extensive optimization. Most higher level language compilers are bulky and the common languages are not particularly well suited (because of their data structures) for writing compilers.

\section{Large primitives}

Definition of good interface languages to each phase of a compiler may offer the best hope of cutting down the cost of compiler production. If a sound structure can be found then improvements can be continuously made to it and the programming debugged; transferability would be the next consideration.

\section{Conclusions}

Compiler designers usually have to meet very rigorous objectives: minimize space, high compilation speed, rapid implementation, low cost, low maintenance costs, good object code, good use of the operating system, good diagnostics and listings, etc. The problem of logistics or resource allocation dominates the design. The challenge is somehow to obtain an optimum and none of the present methods help the designer or guide him in achieving it. For example, more should be known about the design of compilers to operate in a very small memory. COBOL and PL/1 compilers need study. While many of the above problems are common to all of good programming, perhaps starting from the better understood structure of compilers would allow gaining further insight into their solution.

Much research remains to be done and very little of it has been started.

\section{References}

1. Feldman, J. and Gries, D. Translator writing systems. Comm. ACM 11, February 1968, pp. 77-113.

2. Allard, R. W., Wolf, K. A., and Zemlin, R. A. Some effects of the 6600 computer on language structures. Comm. ACM 7, February 1964, pp. 112-119.

3. Allen, F. E. Program optimization. Annual Review in Automatic Programming, 1968.

\subsection{Analyzing large-scale system development}

by

Jules I. Schwartz

The era of large-scale programs began in the mid 1950's with the development of the SAGE system. Since that time, probably of the order of 100 such systems have been built. A significant percentage of these systems have been sponsored by military agencies but in recent years civilian installations have produced such efforts as airline reservations' systems, 123 large-scale time-sharing executives and major business management systems. In Steel (see Reference 21), very large programs are defined as those that require "many times the available primary storage" for code and data and require "more than ten coders" for implementations. These two characteristics are true for all large-scale system efforts, although the number of people involved in most are considerably more than ten. Even in this case, however, the fact that ten "coders" are specified begins to demonstrate the complexities of such efforts. In addition to the team of 
coders some management personnel must be present; and designers, documenters, testers and other support personnel are required. The implementation of these systems is an expensive, difficult undertaking and the history of many of them has demonstrated that there is by no means a complete understanding of the methods and technology necessary to accomplish successfully the aims of these systems.

The stages necessary to implement such systems include definition, design at many levels, production, testing, operation and evaluation. These stages interact and are subject to a substantial number of feedback loops. The organization necessary to carry out such an effort is large and complex, and must contain people with a variety of skills. In analyzing the problems in producing such systems, one could look at any of these phases, and in fact the original intent of this paper was to discuss the subjects of testing and evaluating these systems.

Although recognised to be one of the major problems in data processing, the subject of testing and evaluation is probably one of the least discussed subjects in the literature. It is recognized that close to 50 per cent or more of the production of large systems is devoted to the period of testing. It has also been noted that little, if any, formalization of the process has been developed; and for a variety of reasons, much of the experience has not been described in writing. One reason for this is that some techniques are considered proprietary (although perhaps "embarrassing" is a more likely descriptive term). Also, much of what does exist is imprecise enough to defy formal description.

The author has been involved in the design and checkout of a number of large-scale programming systems. From these experiences, it seems as though one could state principles and procedures which others should follow in the checkout of large-scale systems. Unfortunately, this is not easy to do. The author has the distinct feeling that, to a large extent, his and other experiences were the result of system development done in a style rather like a random walk. Certain techniques are recognized to be of value for producing large-scale systems some of these are referenced in the following. Other techniques are extremely difficult to define. They lack algorithmic interpretation and no methodology really exists.

One of the difficulties in synthesizing available information is that the subject Testing and evaluation" actually covers a wide range. First of all, there is the actual checkout of systems; this includes the checkout of the lowest level of programs through operational testing. During this period, one is trying to verify whether the system is performing inconformity with existing specifications. Then there is the subject of relative testing - that is, can a function be performed better given different hardware or different programming? Included in this area is the subject of bench-mark testing, on which there is a relatively large set of references $(2,3,4,6,9,11,12,13,22$, for example). These articles on bench marking define techniques that will verify or measure the relative throughput, turnaround time or efficiency of one system versus another. The normal problem in large-scale system testing is quite different. In this case, one is given a particular configuration and a major mission to perform. Implementing such a system is normally a major task, and the ability to compare its results with another system is virtually absent.

A third area of evaluation has recently been receiving more attention. This includes the subject of measuring user interest and performance on a particular system. To a large extent the increasing interest in this subject $\mathbf{1 2 4}$ has come about because of the rising interest in timesharing and interactive computing over the last few years. For some reason, prior to on-line or interactive computing, very little concern was given to the user and how he performs with or benefits from various aspects of the system with which he operates. However, with the influx of timesharing systems, a great number of questions have been asked concerning the value of these systems versus the more traditional techniques. Consequently, there have been a number of experiments run to compare the value of interactive and non-interactive computing (see Reference 18).

After some contemplation, it appeared that divorcing the testing from the remainder of the system development task would not be appropriate. New ideas devoted to this area alone are not worth a complete paper at this point, at least with the author's current set of ideas. The entire system development cycle is, or should be, thoroughly integrated. Testing should be a consideration through the entire development. The major problem is the total effort on these systems, of which testing is one part. Many ingredients contribute to the ultimate success or failure of a large-scale system. It seems then that a discussion of these factors would be of more value than a treatise on testing alone. Thus this discussion will proceed on the basis that overall system evolution is the major problem, with testing as one of the major factors. 


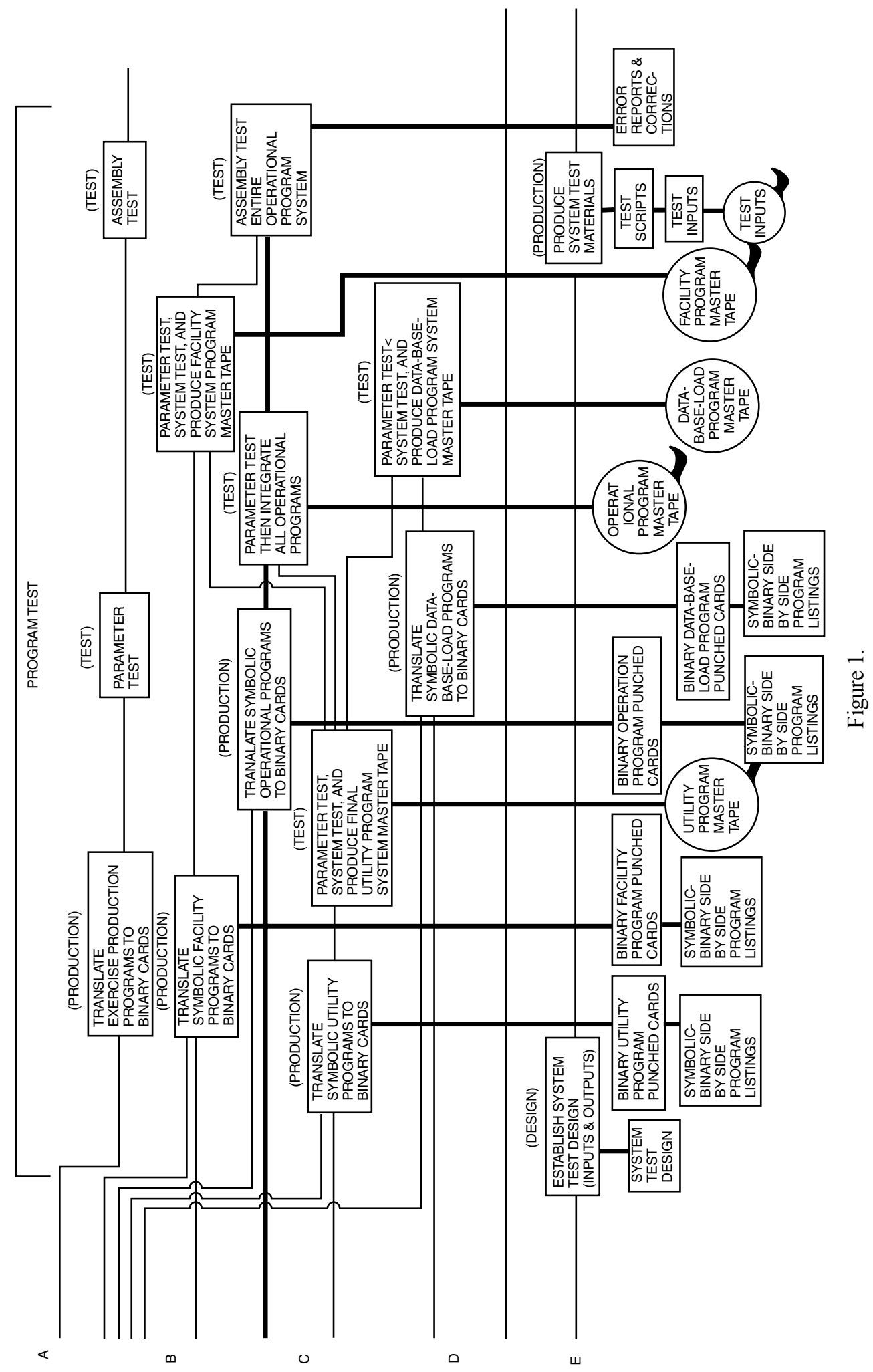




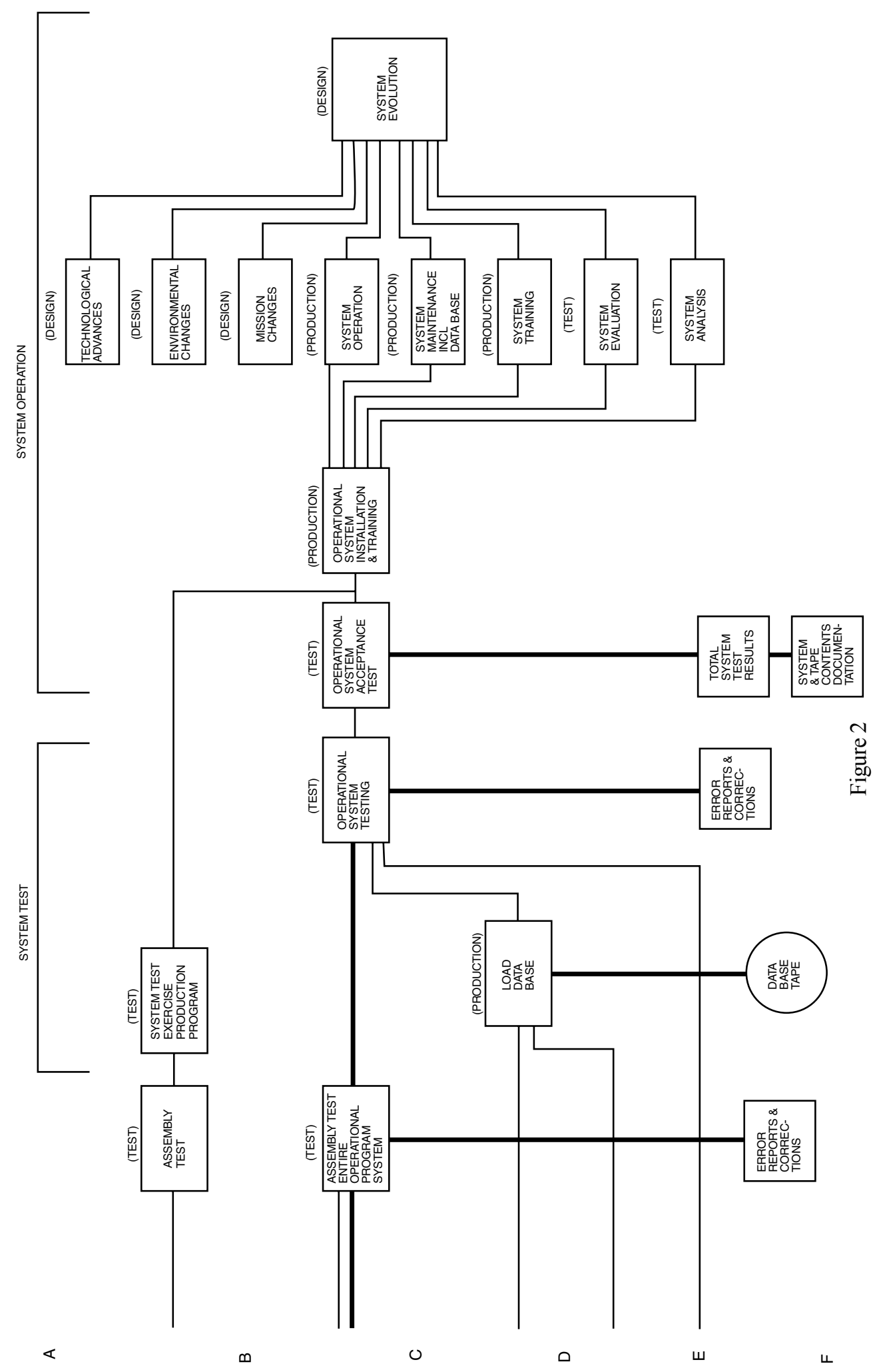




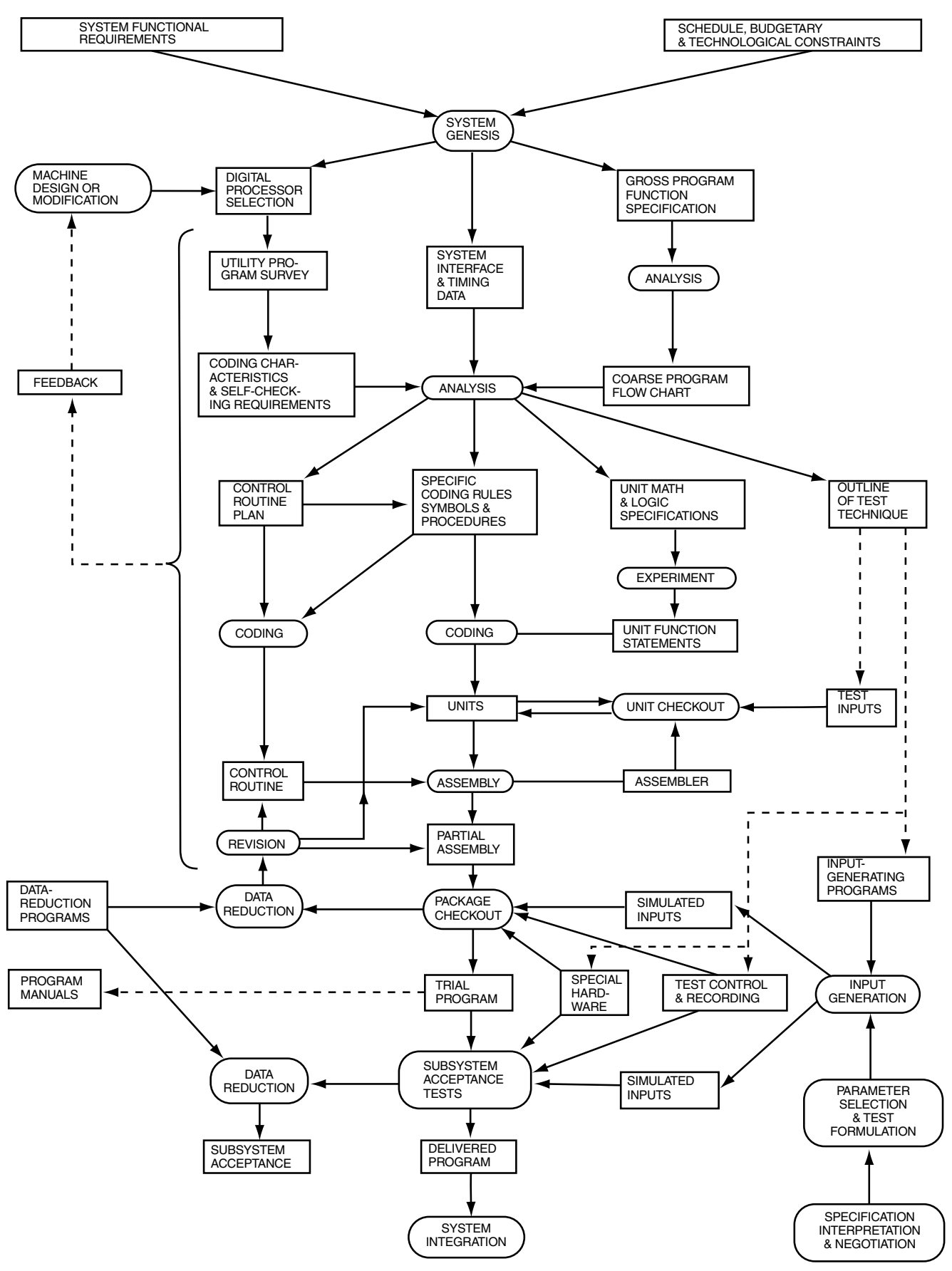

NOTE: Only Major Lines of Influence and Feedback shown. Broken Lines Show Omission of Contributary Processes.

Figure 3 


\section{Experiences with large-scale systems}

Producing a large-scale system is a massive undertaking. Figures 1 and 2 show graphically the typical steps necessary in just the test process for these systems. These Figures (originally published elsewhere*) are discussed in Sackman (see Reference 17) in a chapter on the experimental method in man-machine systems. Sackman points out that throughout the entire system development process, there has been little if any methodology developed. In the area of testing the people involved have had little experience in the use of experimental design and analysis and, consequently, few if any statistically valid attempts at testing large-scale systems are made.

Another description of the system development process is given by Hosier (see Reference 10). Figure 3 is from Hosier and graphically illustrates the complexity of a system development task. Hosier's article, although published in 1965, gives a thorough description of the system development process and difficulties. Among other things, Hosier discusses at some length the need for both stereotyped samples and random tests generated by statistically valid means. Hosier also makes some observations on the administrative problems involved in system development and testing. He points out that the ones who have the primary responsibility for accounting for the status of the schedule are the nonprogrammers, who have "little understanding and less sympathy" for problems in meeting schedules. Their probable attitude towards slippages may be reflected by threats and lectures, rather than by any attempt to see if the schedule, original program design, table design or other factor is at fault. Meanwhile, of course, programmers are in their own world, reluctant to "stop tinkering" (Hosier) with their product. Somebody must be able to distinguish the problems associated with the programmer, design hardware or administrative factors.

There is a considerable body of reference material on techniques for programming and debugging. Whether the technique utilizes CRT, on-line typewriter, memory dump, trace or other device, numerous authors have been able to communicate ideas in this area (References 5 and 7 are two discussions). However, these tend to be largely in the area of individual program production. Rather little literature exists on the checkout of assemblages of components. Even in the area of individual checkout, many weaknesses are recognized. It appears that as the hardware and $\mathbf{1 2 8}$ programming systems have improved, we have about stayed even. That is, it takes about as long to check out programs as in the past, although the programs are now much bigger and more complex. Another interesting and perhaps startling possibility is mentioned in a report by Sackman and Grant (see Reference 19). They describe an experiment comparing debugging in the interactive and off-line environments. They found that, in these tests, the difference between the two environments was approximately a factor of 2 , while the noticeable difference in programmers who participated in the experiment seemed to range up to more than a factor of 20 ! This set of subjects was taken from what was presumed to be a fairly homogeneous, and skilled, organization.

Another experience was reported on informally which further illustrates the confounding nature of these systems. One of the managers of the SAGE program development effort was once asked to recount the experience with the development of that system. He described the history that led to this being one of the largest programming efforts of all time. The early effort was based on experience with a prototype system at MIT. Based on this effort, the SAGE program should have required a reasonable number of people and time. But it soon became apparent that the actual system required substantially — that is, orders of magnitude-more effort than the prototype. Documenters, testers, operational programmers, utility programmers, designers, table experts and interface specialists were needed. All of these required managers at a variety of levels. The managers required help of both administrative and technical nature. As schedules tended to slip, or difficulties were recognized, more people were hired. This, of course, required more management and communication. This cycle continued for several years until many hundreds of people were involved in the programming effort. The program, although considered by most people to be a landmark as well as one of the relatively few successes in large-scale system programming, was delivered later than originally planned and with somewhat less capability than that originally desired. When asked what he would do differently if he had to do a system like this again, the manager, after some period of reflection, said he would hire twelve good people to do the whole job. Outside of that, he could not think of much he would have done differently.

Another large-scale system was produced using much the same concept as SAGE: a massive effort mounted rapidly and requiring a high degree of organization and management talent. This system received considerable publicity when a high-ranking dignitary made the observation that the development of this system cost $\$ 35$ an instruction. This amount was presumably high or else, unfortunately for the system's producers, this was the first time anybody ever

* Willmorth, N. E. System Programming Management, TM-(L)-2222, System Development Corp., Santa Monica, Calif., 1965.

\section{NATO SOFTWARE ENGINEERING CONFERENCE 1969}


labelled a system's cost in this way. It might be pointed out, however, that in this case probably several hundred manyears were spent on the system before the operational programs were begun. That is, it required considerable time to reach an understanding of what the system was supposed to do, after work on it was begun.

Although the field of large-scale system programming is barely fifteen years old, there are many stories like the preceding. Few systems have been complete successes in that they were on time, within the original budget estimates and contained all the expected capabilities on the originally planned equipment. The reasons for this are many but it is clear that one factor is paramount. This factor is people. Although the manager of the SAGE effort who stated that he would like to do the job with a dozen people was probably exaggerating, he was expressing a frustration felt by all managers engaged in these efforts.

\section{The key element}

A large-scale system effort requires a substantial number of people. Some of the things we know about these people are:

1 They don't know the complete operational problem.

2 They communicate badly.

129

3 Within a group of programmers, there may be an order of magnitude difference in capability.

4 Good programmers are creative. They are creative in situations that may not require creativity.

5 Non-programmers involved in these systems can't understand programmers. Put in a position of responsibility, however, this does not normally restrain them from hiring more.

6 It is extremely difficult to find out from people how a job is really progressing. Most programmers are optimistic by nature. Most managers are gullible. Our methods of prediction are incredibly bad.

7 The people involved in large-scale system testing are lacking in knowledge of methodology which enables them to state with confidence that a system is checked out.

$8 \quad$ No one person understands the whole system or its status.

The problems and some recommendations

One can conclude, then, that the large-scale system development process is complex, expensive and uncertain to a significant degree because large groups of people are utilized who are either not equipped to do their job or who may be doing the wrong job. Normally, of course, one doesn't recognize the difficulties of a systems development until the testing phase. Consequently, the people involved in this area bear the wrath of those who, until that time, assumed things were going well. In fact, the situation might have deteriorated quite badly by the time it got to the testing stage. It seems clear, however, that many of the difficulties are recognizable and perhaps some effort can improve the situation. In the preceding, some points were made concerning the people involved in producing these systems. Since people are the major resource and problem in the production of these large systems, a more detailed discussion of these points should be developed.

\section{People don't understand the complete operational problem}

In the early stages of the development of these systems, it is sometimes unclear that even the "customer" knows what he really wants. Of course, some subset of the customer group might have most of the information but the communication of these ideas is not easy. However, many venture into these systems quite unafraid, willing to figure it out after the work starts. Before allowing the build-up of the people and equipment necessary to implement the system, the customer and producer should be clear as to the mission to be performed. At least some subset of the producing organization should be operationally oriented. In addition that difficult-to-find operationally oriented but computer knowledgeable person should be available. A measure of understanding is the ability to define tests for the system at this early stage. The test design should continue from the first analysis until the system completion. It has recently been argued by some that it will be impossible to test adequately the programming of a currently proposed major procurement. If true, this foresight can be of considerable value. In any case, no system should be produced without thorough comprehension of the operational problem and the technique for its testing.

During the past several years, the Air Force (and recently the Department of Defense) has adapted management concepts to the problem of computer program acquisition. These Air Force 375 Management Concepts as applied to

\section{NATO SOFTWARE ENGINEERING CONFERENCE 1969}


software are based on the decision that a computer program can be defined as a Computer Program Contract End Item (CPCEI). Described in some detail in References 14, 15, 16, and 20, the procedures detailed in this system include the definition of specifications and review procedures which assist considerably in assuring that the operational requirements are defined. before the system implementation begins. It also assures that test specifications are written concurrently with the system definitions.

\section{0}

People communicate badly

Communicators are needed. This includes people who can define the required system documentation. Then management must see to it that the documentation gets written. Frequently this is one of the chores left to programmers, who are in the main notoriously reluctant as well as not very skilled at writing. There are, however, individuals who understand programming and like to write. These people must be isolated and assigned the task of working with the operational and programming people to produce a complete series of operational, utility and test program specifications.

\section{There is a great difference in programmers' capabilities}

We have all recognized this to some degree. Perhaps, however, we have been surprised by the quantitative aspects of this problem. Part of the difficulty is the fact that programmers are different in what they do well and in their styles of work, as well as in how well they perform. There are the extremely fast coders who check out for a long period. There are also those who code with great care and rarely go to the computer until the deadlines are near. The latter cause particular strain on managers. There is some possibility of measuring progress once the program is running. But in the long run it is not clear that either style is better. This difference in programmers, however, does make it much more difficult to schedule and to measure progress in the early phases.

The key is, of course, the manager. He must be able to recognize whether progress is adequate at any time. If it is not he must take corrective action. This may be the adjustment of priorities, the shifting of personnel or the sometimes painful removal of a person. If a manager is willing to recognize the fact that some programmers are bound to be considerably less capable than others, and he constantly tries to filter out the less capable set, he is much more likely to succeed than by adhering strictly to procedural techniques.

\section{Good programmers are creative}

The art of programming requires creativity. The nature of code is such that there is an almost unending set of possible improvements. There are probably an infinite number of ways to program any problem. Few programs exist that seem to satisfy all the possibilities that one might imagine. All these factors further complicate the problem of system production. The manager must constantly be on the alert for creation beyond the bounds of discretion. Testing can be delayed considerably by those who are changing the thing being tested. Decisions as to satisfactory performance must be made and the line drawn, so that further "improvements" are forbidden.

This is a particularly sensitive area. One must limit imagination in some sense, yet try to keep a technical environment that will encourage good people to remain on the job. This is a somewhat perplexing problem in large-scale system development anyway, since these are usually large team efforts and individual contributions have to be made subservient to the total job. There are various techniques for directing this kind of effort. One can be called the "top-down" approach. Using this technique, the system is designed and first level specifications are written at the top level. At each succeeding level the specifications are reviewed and more detailed specifications are written. The specifications eventually include descriptions of programs that are to be written at the next level. These, in turn, might generate the requirement for more routines at lower levels. In sum, then, the programmer never writes the specification for the program he is coding, and the person at a particular level knows rather little about the total system or even the characteristics of other programs at the same level. In the extreme, the person at a given level does not know who prepared the specification he is using. This technique has the advantage of clearly delimiting the requirements at any level and forcing relatively clear thought $\mathbf{1 3 1}$ processes throughout the production cycle. It presents co-ordination problems when difficulties that arose at some previous level are detected. One can assist this type of process by forc-

\section{NATO SOFTWARE ENGINEERING CONFERENCE 1969}


ing the specifications at any level to include the description of validation tests at the next lower level, although these could be written by someone other than the specification writer.

Another common occurrence in large-scale system development is due partially to programmers' urge to create, partially to the usual start-up period before operational programs are ready for checkout, and perhaps somewhat to the recognized complexity of systems. For most new systems, it has been found that totally new and advanced support tools are needed. These utilities are, in themselves, quite sophisticated and usually a large step over the previous versions for other systems. They are the creative programmer's dream; but they are subject to the same developmental difficulties as the operational system. They are usually not ready by the time operational programs need checkout. They use the operational program for their own checkout. This has obvious implications for the operational program. It seems as though a somewhat less advanced utility system would be in order in most cases.

There are non-programmers involved in most aspects of the system development process

They may be experts in their respective fields but they don't necessarily understand the transformation of their ideas to the computing process. The non-programmer can state in operational terms the function to be performed. He can be particularly valuable in writing test criteria. He serves as an excellent liaison with the customer. He can give unbiased commentary on the human interface with the system. He has difficulty, however, in understanding the feasibility of a particular recommendation. Things which may appear to be of widely disparate difficulty to the programmer may appear to the non-programmer to be of equal difficulty, or even the inverse of the programmer's ranking.

Non-programming managers usually have the option of trusting the programmer in situations or of trying to inflict their judgment on the programming personnel. Either course without adequate understanding is dangerous. As noted before, programmers have various degrees of skill, so that complete faith in all of them is precarious. On the other hand, the nonprogrammer's judgment as to the proper course to take in a given case is not necessarily correct. The only realistic possibility lies with the programming manager. A programming manager who understands the situation and its potential and can communicate with the non-programming personnel is essential. He must represent the programming group. He must negotiate the possibilities and the course of action to be taken. He must then communicate decisions and courses of action to the programming group in their terms.

\section{It is extremely difficult to judge the actual status of a system at any given time}

A variety of documents have been written on the subject of planning and measuring progress of large-scale systems. Milestone schedules, PERT charts, progress reports and various other techniques are used. But a number of practical situations exist that make precise statements of progress and predictions very difficult.

First there is the relatively obscure nature of coded programs prior to checkout. To know whether the code that is being written accurately reflects the specifications requires detailed analysis, which is more than many managers can handle. One method of checking consists of extremely thorough production and review of various steps prior to coding. Successively detailed levels of specification, including flow diagrams, will create a situation where the coding is a relatively straightforward translation as opposed to an invention. These steps require careful analysis, 132 considerable co-ordination and much time: probably much more time before checkout begins than a relatively brief planning period followed by coding. The total elapsed time through checkout may or may not be greater in either situation. To a large extent, the success or failure of either technique depends on the programmers involved. Some can, with relatively little preliminary effort, produce accurate code and know precisely how far along they are. Others are not so trustworthy. In any case, tracking the effort at this state requires an organization where the lowest-level manager has teams small enough to permit thorough inspection of the ongoing work.

During the checkout of sub-programs, it is somewhat easier to understand the status than during the coding period. This assumes, of course, that reasonably precise tests have been specified for this level. Verification of correct results is only one part of the testing at this phase, however. A well designed system should have time and space constraints included for all modules. This is obviously critical in systems that have operational constraints but can also produce catastrophic results if no measures are made at this time on systems that are not absolutely constrained. A number of these latter systems have "worked" but in what amounts to impractically slow times. 
Most people are reluctant to admit defeat. This is reflected in their inability to recognize that some schedules may be slipping. Until the actual deadline is passed, there is always the "hope" that things will somehow come out all right even though they are temporarily not in line. Of all the system manager's difficulties, this is probably one of the worst. Schedules do slip, whether because of inaccurate prediction, poor programming or other phenomena. These slips, or impending slips, must be recognized and actions must be taken. A particular component's slip may not have a deleterious effect on the rest of the development. With good planning, this can be recognized and corrections can be made locally. But other slips or excessive capacity requirements can have serious ramifications affecting the entire system. These must be recognized and effective measures taken immediately. Reactions taken early are considerably more valuable than later steps taken in panic.

The Air Force 375 procedures make a significant attempt to define the milestones required for the measurement of progress. They do serve as excellent signposts of progress while a system is in production. Of course, these measures assist in the measurement of progress but do not offer alternate courses of action when required. They alert people that things have gone awry earlier than would have been possible without these procedures.

There is little methodology used in testing or system development

Characteristically, large systems deal with great masses of information. There is not enough time to verify "all cases" at every level. There has been a significant amount of study devoted to the problem of statistically valid sampling. However, little use of this field is made in the testing of large systems. The people involved in testing are usually the same ones who developed the programs, until the final stages of the system production. They are usually unconsciously biased, are anxious to get through with the job and are not well qualified in the field of testing. These personnel, although able to execute the tests, should not be the test designers. Personnel who understand the methodology and system requirements should be assigned the job of writing test specifications and should verify the results of all tests. This testing organization should work with the implementation personnel only enough to understand the system structure sufficiently to prepare the test specifications. For this purpose, as well as for the rest of the system development, a total system implementation philosophy must evolve.

Figure 4 is a simplified description of a large-scale system. There are several approaches to the design and implementation of such a system. There is the "algorithmic" approach, where one is concerned with the 134 calculations, decisions, control mechanism, interrupt mechanisms and probably directly, with the devices at either end of the system. The system is structured around these areas. The algorithms are designed and the data structures grow to fit the need of the algorithms. In a sense, the system is built from the inside outward.

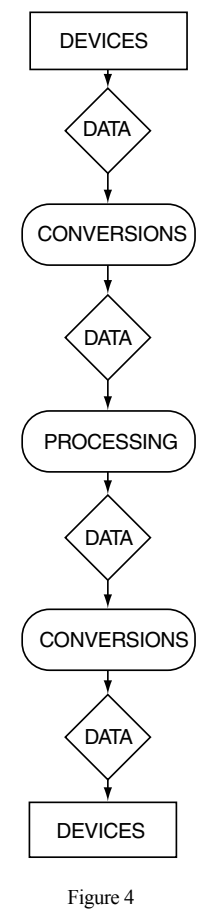


Another approach is the building of the system around the data. In the "data" approach, one conceives of the system as a set of elements, tables and files in various states. The states vary from raw, probably well-defined structures associated with the devices at either end to completely designed problem oriented structures that are associated with the processing.

In actuality, of course, most systems are produced using both techniques to a certain degree. It is impossible to develop a system using either approach in its pure form. However, it is probably true that a large number of systems are produced with the emphasis on the "algorithmic" approach, whereas it seems more appropriate to emphasize the data in the development of a large system.

The reasons for the usual emphasis on function rather than content are probably largely due to the mathematical and scientific backgrounds of the majority of people involved. Most people tend to think in terms of the functions involved in a process and assume rather restricted characteristics of the data. This is also demonstrated by the limited nature of many programming languages, FORTRAN and ALGOL being excellent examples, where data definitions beyond arrays of real numbers have been lacking. Some languages, like JOVIAL, COBOL and PL/1 have had considerably more emphasis on the data approach to problem solving. (JOVIAL has frequently been called a language for Command and Control. Perhaps a better description would have been a language for System Programming.)

There are various reasons why the building of a system around its data at different stages is advantageous.

The description of the data provides an unambiguous statement of the system at each stage. By clearly defining the elements and their values, one must understand and convey the essentials of the process. In addition, one can create a reasonably efficient system by thoroughly analyzing the dataflow through the components, eliminating redundancies, excessive paths and missing paths. The data definitions serve as an excellent vehicle for specifying the operating components of the system. The definition of each operating component can consist primarily of its input and output data.

In defining the data, the system designer must understand the capacities required for the various stages of system operation. Allocations of space and positions for the data can be made independent of the algorithm involved in processing them. This prevents the construction of algorithms which work well only with data that may be incompatible with system requirements.

The system test tools can be built systematically around the data. A scheme for testing must be developed which lends itself to automation. It is desirable to have the technique apply to all levels of system checkout even when all the input and output devices haven't been installed. Also; it is imperative that a component, or subsystem, once checked out, can be guaranteed to work in the total system environment. These requirements can be met through a checkout scheme oriented around the system data description.

The data types for large-scale systems are quite varied and can be structurally organized in a variety of ways. For example, the data as input from devices or the formatted data needed for output devices may be relatively difficult to describe in higher-level program language terminology. Frequently, they are strings of information defined more in terms of what the hardware can produce as opposed to design for ease of programming and conversion. The data used by the central processing programs can be designed much more logically and within the scope of at least some programming languages. In either case, it is possible to define the information with the technique that permits the naming of the elements and the association of specific data types with each element. In some cases, the 135 data is strictly numeric and in, perhaps, standard array form. In other cases, it is logical: where specific bit patterns mean certain states. In other cases, there's alphanumeric information. Some elements are frequently found to occupy more than one computer word and, for large-scale systems particularly, some elements occupy only parts of words. Some elements are simple in that they occur only once and others are repetitive.

The major part of the system should be the definition of the variety of element and file descriptions. Once this is done, specifications for programming and checkout of the system can be done in a fairly systematic manner. Specification for each component includes the list of elements that are input to it and the list of elements and files which it should output and the corresponding states for the output given certain inputs. The checkout of each component, then, can be done in terms of the input elements and the associated output. Likewise, subsystems or groups of components can work with element descriptions. 
Since a large-scale system is actually composed of many sub-components and systems, it is important that the data used to verify individual pieces is consistent through all tests. One technique which was originally used in the development of the SAGE system still seems to hold the greatest potential in this area. The technique consists of defining for the entire system all data that will be used by more than one component. These data descriptions, including their types and structures and capacities, are entered into a single data definition table, which applies to the entire system. This table was called, in the SAGE system, the Communication Pool. The advantages of a centrally defined data set are several. First of all, it provides uniformity throughout the system during the checkout phase. Secondly, it permits the devising of checkout tools that are data oriented. Third, given that a compiler or assembler exists that is sensitive to the data description, it permits the programming of a system that requires minimal change when the data definitions are changed-a common problem in the development of large systems.

Given the existence of a centrally assembled data definition such as the Communication Pool, one can now devise a hierarchy of system checkout tools. First of all, there are relatively simple table simulation programs which permit the definition of the value for the elements required by each component. Thus, this component level simulation program can automatically produce environments for each component during check out. At succeedingly higher levels, simulation programs are permitted which again deal in terms of the element names and their natural values, as opposed to hand-input computer oriented values. For output of tests, similar kinds of format routines can be produced which take a given binary or computer form of the file structures and produce formatted output at the end of a run, using the central data definition as the source for formatting. Considerably more automation can be added to the system by table generation routines and routines that compare outputs of runs with prespecified expected results. It is here, of course, that valid sampling techniques should be used.

\section{No one knows the whole system}

We frequently hear of successful implementations of complex systems by one or several people. These small-scale efforts are totally in the control of a small set of people. By virtue of this, all planning is integrated, reasonably optimum solutions can be conceived over the whole system and reaction to contingencies may be developed within the total system context. We are concerned here, however, with systems that require, or at least utilize, considerably more than several people. The program is large and probably fragmented into a number of quite distinct logical functions. The hardware complex is usually large and not standard. System specifications must evolve through interaction with non-programming personnel. Stringent demands on operating capabilities and capacities generally exist. Testing must proceed with extreme caution. Problems of adaptation to new environments and $\mathbf{1 3 6}$ evolving situations must be accounted for throughout the system development. If only by virtue of the variety of personnel involved, the largescale system development must use an organization considerably larger and more complex than the small efforts. One effect of this situation is the diffusion of information. Actions must be taken and problems diagnosed by people with either a very detailed knowledge of only one part or those who have a rather general "big picture" view of the situation. Those responsible for major decisions must weigh the not always completely accurate advice of many people.

From this plethora (or paucity) of facts such decisions as changing scope, modifying schedules, hiring or firing people or reconfiguring the hardware must be made. Managers must understand and have very trusted, competent subordinates.

\section{Summary}

In summary, large-scale system development is a "people" problem. There are procedures, techniques and systems which can assist in the development of a system, but they are aids only to the personnel involved in carrying out the implementation. The known techniques describe the documentation, provide a philosophy for production and serve as guide-lines for automating the technique of checkout at the various levels of system checkout. They do not solve some of the more intangible problems such as accurately measuring progress, developing precise testing methodology and making serious contingency decisions.

Considerably more research must be done in understanding capabilities of individual programmers. Even in relatively homogeneous groups there is a great spread in capability. People experienced in testing and experimentation should be applied to the problems of system testing. People should be assigned full time to the data analysis, design and change areas.Also, the support system should be built around the data at all levels. Studies should be made of the qualities of management personnel necessary to guide complex developments such as these to a successful conclusion. Choosing the lowest level manager is one of the most important decisions in such efforts. Analysis of the organization required to

\section{NATO SOFTWARE ENGINEERING CONFERENCE 1969}


specify, produce and test such systems needs to be done in order to better specify the types, numbers and levels of the people needed in such efforts. Until all these things are adequately accomplished, large-scale system development will remain an art which is more dependent on luck, hard-to-define experience and tenacity, rather than on well-defined principles of production.

\section{References}

1. Adams, W. I. and Federico, P. R. Cadfiss test system computation and data flow integrated subsystem tests, Proceedings of the 19th National Conference, Association for Computing Machinery, Philadelphia, August 1964, pp. A2.3-2/A2.3-7.

2. Arbuckle, R. A. Computer analysis and thruput evaluation, Computers and Automation, January 1966, pp. 12-19.

3. Buckley, Fletcher J. Estimating the timing of workload on ADP systems: an evaluation of methods used, Computers and Automation, February 1969, pp. 40-42.

4. Calingaert, Peter System performance evaluation: survey and appraisal, Communications of the ACM, 10, No. 1, January 1967, pp. 12-18.

5. Chapin, Ned (Dr.) Logical design to improve software debugging — a proposal, Computers and Automation, February 1966, pp. 22-24.

6. Estrin, G., Hopkins, D., Coggan, B., and Crocker, S. D. SNUPER computer-a computer instrumentation automaton, SJCC, 1967, pp. 645-656.

7. Halpern, Mark Computer programming: the debugging epoch opens, Computers and Automation, November 1965, pp. 28-31.

8. Hext, Jan B. (Dr.) Recovery from error, Computers and Automation, April 1967, pp. 29-31.

9. Hillegass, John R. Standardized benchmark problems measure computer performance, Computers and Automation, January 1966, pp. 16-19.

10. Hosier, W. A. Pitfalls and safeguards in real-time digital systems with emphasis on programming, IRE Transactions on Engineering Management, EM-8, pp. 99-115.

\section{7}

11. Joslin, E. O. Application benchmarks: the key to meaningful computer evaluation, Proceedings of the ACM 20th National Conference (Session 2: Evaluation and Performance of Computers), 1965, pp. 27-37.

12. Joslin, Edward O. and Aiken, John J. The validity of basing computer selections on benchmark results, Computers and Automation, January 1966, pp. 22-23.

13. Karush, Arnold D. Benchmark analysis of time-sharing systems, System Development Corporation, Santa Monica, California, 31 March 1969.

14. Liebowitz, Burt H. The technical specification—-key to management control of computer programming, in AFIPS Conference Proceedings, Thompson Book Co., Washington, D.C., 1967, Vol. 30, pp. 51-59.

15. Piligian, M. S. and Pokorney, J. L. Air Force concepts for the technical control and design verification of computer programs, in AFIPS Conference Proceedings, Thompson Book Co., Washington, D.C., 1967, Vol. 30, pp. 61-66.

16. Ratynski, Milton V. The Air Force computer program acquisition concept, in AFIPS Conference Proceedings, Thompson Book Co., Washington, D.C., 1967, Vol. 30, pp. 33-44.

17. Sackman, H. Computers, system science and evolving society, John Wiley \& Sons, Inc., New York, 1967.

18. Sackman, H. Time-sharing versus batch-processing: the experimental evidence, Proceedings of the Spring Joint Computer Conference, 1968.

19. Sackman, H. and Grant, E. E. An exploratory investigation of programmer performance under on-line and offline conditions, IEEE Transactions on Human Factors in Electronics, HFE-8, No. 1, March 1967.

20. Searle, Lloyd V. and Neil, George Configuration management of computer programs by the Air Force: principles and documentation, in AFIPS Conference Proceedings, Thompson Book Co., Washington, D.C., 1967, Vol. 30, pp. 45-49.

21. Steel, T. B. (Jr.) The development of very large programs, in Proceedings of IFIP Congress 65, Kalenich, Wayne A. (Ed.), Spartan Books, Inc., Washington, D.C., 1965, Vol. 1, pp. 231-35.

22. Totaro, J. Burt Real-time processing power: a standardized evaluation, Computers and Automation, April 1967, pp. 16-19. 


\title{
7.14 Toward a programming laboratory
}

by

Warren Teitelman

\begin{abstract}
This paper discusses the feasibility and desirability of constructing a "programming laboratory which would cooperate with the user in the development of his programs, freeing him to concentrate more fully on the conceptual difficulties of the problem he wishes to solve. Experience with similar systems in other fields indicates that such a system would significantly increase the programmer's productivity.
\end{abstract}

The PILOT system, implemented within the interactive BBN LISP system, is a step in the direction of a programming laboratory. PILOT operates as an interface between the user and his programs, monitoring both the requests of the user and the operation of his programs. For example, if PILOT detects an error during the execution of a program, it takes the appropriate corrective action based on previous instructions from the user. Similarly, the user can give directions to PILOT about the operation of his programs, even while they are running, and PILOT will perform the work required. In addition, the user can easily modify PILOT by instructing it about its own operation and thus develop his own language and conventions for interacting with PILOT.

Several examples are presented.

\section{8}

\section{Introduction}

The research described in this paper focuses on the programmer's environment. This term is meant to suggest not only the usual specifics of programming systems and languages but also such more elusive and subjective considerations as ease and level of interaction, "forgivefulness" of errors, human engineering, and system "initiative". (The anthropomorphism is deliberate.) In normal usage, the word environment refers to the aggregate of social and cultural conditions that influence the life of an individual. The programmer's environment influences, to a large extent determines, what sort of problems he can (and will want to) tackle, how far he can go and how fast. If the environment is "cooperative" and "helpful" then the programmer can be more ambitious and productive. If not, he will spend most of his time and energy "fighting" the system, which at times seems bent on frustrating his best efforts.

One immediate goal to strive for is an environment comparable to that found in the well designed laboratory of the physical sciences. Such a laboratory usually contains equipment for many applications as well as facilities for designing and building new apparatus, or adapting that already present. In a large (well-funded) installation, the researcher will also often have available assistants for performing the routine tasks. For example, a chemist might simply request an analysis of a sample, and not have to itemize each step in the process. This type of assistant and assistance frees the researcher for problems more worthy of his attention.

Computer based systems have been constructed that create this type of laboratory environment for certain well-defined areas, e.g., mathematics (see References 3, 5 and 6), design of electronic circuits (see Reference 7), and generalized graphical design (see References 4 and 8), such as for aircraft, automobiles and bridges.

These systems are organized to allow the computer to perform the routine work (where routine is a function of the sophistication of the system), while the user guides and directs the process at a relatively high level. For example, in the mathematical laboratory developed by William Martin (see Reference 5), the mathematician interacts with the computer by asking questions or making requests. The system employs graphical input and output (light-pen and display) to allow the mathematician to operate in an environment that closely resembles the pencil and paper with which he is already familiar. For output, the display utilizes subscripts and superscripts and observes the conventions concerning physical size, grouping, and placement of sub expressions which mathematicians have adopted to make it easier to read and comprehend mathematical formulae. For input, the mathematician can communicate directly with the computer via the light pen, either by writing new expressions, or by pointing to old ones or portions thereof.

In a typical case the user might be trying to find the solution of a differential equation. On the screen are displayed one or two equations, while the user has in his head the name of several other expressions or partial results already studied and filed away. The user decides to perform an action such as substituting a displayed equation, solving it for 
some variable, expanding some sub expression in a certain way or perhaps asking to see something else. He makes the request using a combination of lightpen and keyboard signals. These are encoded and transmitted to the system where the appropriate routines compute or retrieve the required new expressions and transmit them back to the display routines which then compile and display the desired new picture.In this way, the user can perform in a few minutes a long and involved analysis which, assuming he $\mathbf{1 3 9}$ did not make any mistakes or lose track of what he was doing, might otherwise take him many hours.

This paper describes a step in the direction of such a laboratory for programming and programmers: the PILOT system. As with the mathematical laboratory, the goal is to allow the computer to perform the routine tasks while the user, in this case a programmer, is left free to concentrate on the more creative aspects of his problem, which is the writing and debugging of a program.

Most of the previous efforts aimed at improving the environment of the programmer have concentrated on providing and improving packages, such as editors, compilers, trace packages and display routines. While a good deal of effort has been devoted to such facilities in the design of PILOT, the basic innovation of the PILOT system is the emphasis placed on the problem of making changes in programs. The reason for this emphasis is that making changes in programs is the task that occupies most of a programmer's time and effort, from the early stages in the development of a program, when his work consists primarily of correcting syntactical and simple logical errors in individual subroutines, to the final stages when the programmer makes the type of logical and organizational changes that affect many different parts of his program.

The problem of making changes to the PILOT system itself is handled as a special case of the problem of making changes to programs in general. Since PILOT is designed to facilitate making changes in programs, its tools and techniques can be applied directly to itself in what is essentially a bootstrapping process. The user can thus easily introduce new tools and/or modify existing ones to suit his own methods and problems. In short, the user can tailor the performance of the system to suit himself. Furthermore, PILOT is designed with this in mind, so that it can co-operate with the user during this phase of the development.

\section{The PILOT system}

PILOT is implemented in the LISP programming language at Bolt, Beranek and Newman Inc., Cambridge, Massachusetts (see Reference 1). Although there is a PILOT subsystem in LISP, all of the features and tools described in this paper were incorporated directly into the BBN LISP system once their usefulness was established, and are now in general use by the entire community of LISP users. It is thus more meaningful to view PILOT as a conceptual system, a philosophy of design. It is this philosophy that we are trying to impart in the hope that it may prove useful in the design and construction of systems in other languages.*

\section{Automatic error correcting}

The initial stages in the implementation of a large program are usually devoted to the writing and debugging of independent component routines. Only after these have been checked out, at least superficially, can the programmer begin to assemble the program and check for inter-routine problems. However, before the programmer can even begin to debug a routine, he must first get it to run, i.e. eliminate those syntactical and/or simple logical errors that cause complaints from the language or system in which he is operating. Facilitating the correction of these lowest level errors 140 would improve the efficiency of debugging by allowing the programmer to proceed directly to higher level problems.

From the user's standpoint, clearly the best of all possible solutions would be for the system to correct these low level errors automatically and continue with the computation. This is not far-fetched: a surprisingly large percentage of the errors made by LISP users, e.g. misspellings and certain types of parenthesis errors, are of the type that could be corrected by another LISP programmer without any information about the purpose or application of the LISP program or expression in question.** If these corrections were performed automatically by a program that was called only

\footnotetext{
* LISP is especially suited for implementing a system such as PILOT because of the ease with which LISP programs can be treated as data by other programs. This capability is essential for creating tools which themselves will create and/or modify programs, an indispensable feature of a programming laboratory.

** We conjecture that this is also true in other languages.
} 
after an error occurred in the execution of a LISP program, it would in no way detract from the performance of the LISP system with debugged programs. Thus the efficiency of the error correcting program would not be a critical factor in its usefulness.

A primitive program which corrected certain types of spelling errors was implemented in PILOT and users were encouraged to experiment with it and comment on its features. As a result of this experience, we discovered that in order to be acceptable to users:

1 The program must have a measure of how certain it is about the nature and correction of a mistake, and use this measure in determining the amount of interaction with the user.

2 The program must be able to distinguish between significant and trivial corrections, and to be more cautious, i.e., more interactive, about correcting the former.

3 The user must be able to specify to the program his degree of confidence in its ability to correct his mistakes, as reflected by the amount of interaction he desires.

4 The user must be able to interrupt and/or abort any attempted correction.

5 The user must be able to disable or overrule the entire correcting program whenever he wishes.

With these criteria in mind, a more sophisticated set of error correcting routines were implemented. These routines make up the DWIM package (Do-What-I-Mean). The following output is representative of the kind of corrections and flavour of interaction of DWIM. User input is preceded by an arrow $(\leftarrow)$.

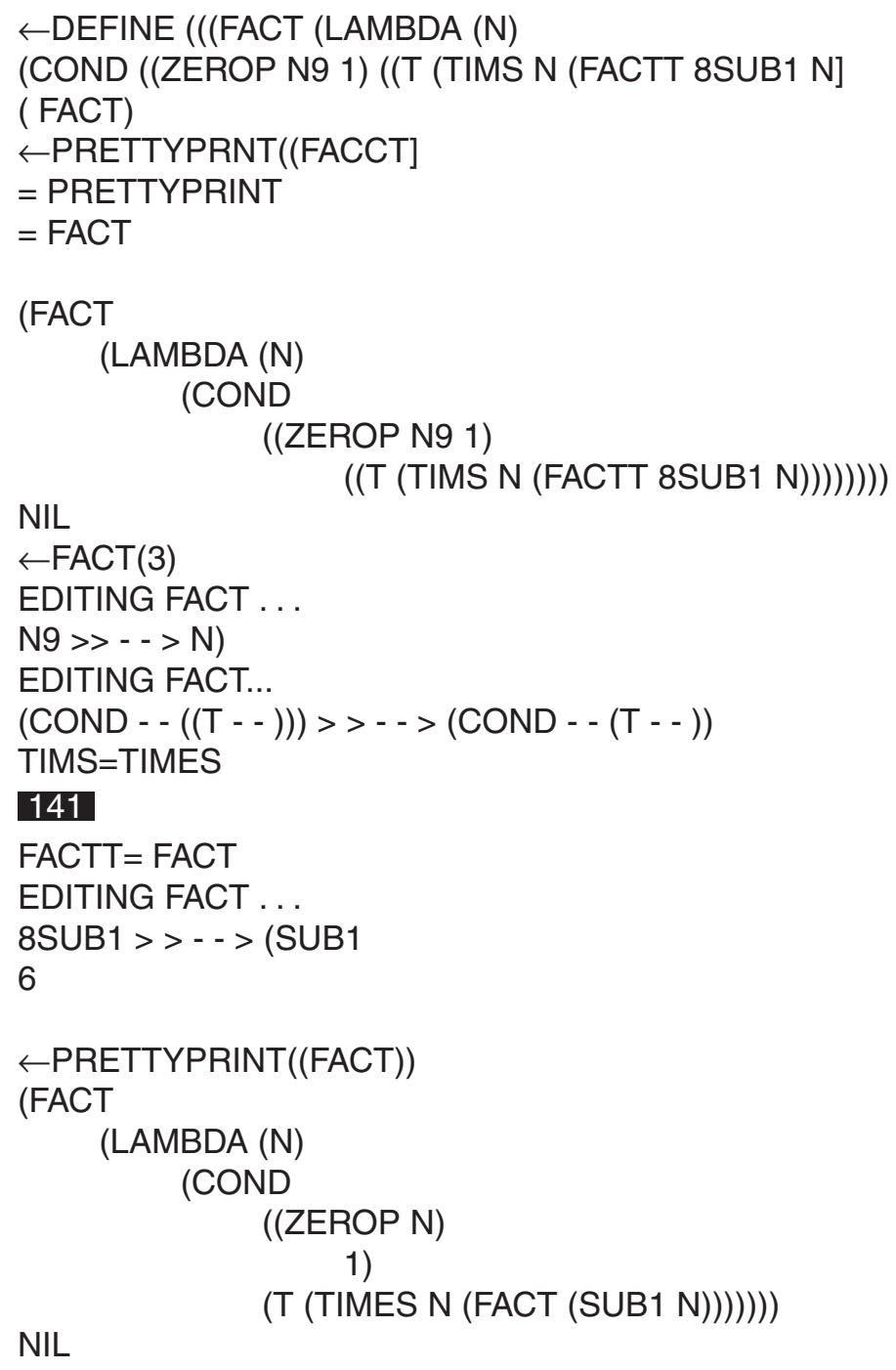


In this example, the user first defines a function FACT of one argument, N. whose value is to be N factorial. The function contains several errors: TIMES and FACT have been misspelled. The 9 in N9 was intended to be a right parenthesis but the teletype shift key was not depressed. Similarly, the 8 in 8SUB1 was intended to be a left parenthesis. Finally, there are two left parentheses in front of the $\mathrm{T}$ that begins the second clause in the conditional, instead of the required one.

After defining the function FACT, the user wishes to look at its definition using PRETTYPRINT, which he unfortunately misspells. Since there is no function PRETTYPRNT in the system, an UNDEFINED FUNCTION error occurs, and the DWIM program is called. DWIM invokes its spelling corrector, which searches for the best possible match in a list of functions frequently used (by this user). Finding one that is extremely close, DWIM proceeds on the assumption that PRETTYPRNT meant PRETTYPRINT, informs the user of this, and calls PRETTYPRINT.

At this point, PRETTYPRINT would normally print (FACCT NOT PRINTABLE) and exit, since FACCT has no definition. This is not a system error condition, but the DWIM facility is not restricted to just system error conditions. DWIM also modifies selected system functions, such as PRETTYPRINT and DEFINEQ, to make them co-operate more closely with the user: DEFINEQ is modified (by ADVISE, to be described later) to note any new functions defined by the user, and add them to the spelling list of user functions. Similarly, PRETTYPRINT is modified so that, when given a function with no definition, it calls the spelling corrector. Thus, PRETTYPRINT determines that the user wants to see the definition of the function FACT, not FACCT, and proceeds accordingly.

The user now calls his function FACT. During its execution, five errors are generated, and DWIM is called five times. At each point, the error is corrected, a comment made of the action taken, and the computation allowed to continue as if no error had occurred. Following the last correction, 6, the value of FACT(3), is printed. Finally, the user prints the new, now correct, definition of FACT.

In this particular example, the user was shown operating in a mode which gave the DWIM system the green light on all corrections. Had the user wished to interact more and approve or disapprove of the intended corrections at each stage, he could have operated in a different mode. Or, operating as shown above, he could have at any point aborted the correction, or signalled his desire to see the results of a correction after it was made, by typing a on the teletype.

We have found from our experience with DWIM that most users are quite willing to entrust the program with the correction of errors, although each different user may want to operate with a different "confidence factor", a 142 parameter which indicates how sure DWIM must be before making a correction without approval. Above a certain user-established level, DWIM makes the correction and goes on. Below another level, DWIM types what it thinks is the problem, e.g., DOES PRTYPNT MEAN PRETTYPRINT?, and waits for the user to respond. In the in between area, DWIM types what it is about to do, pauses for about a second, and if the user does not respond, goes ahead and does it. The important thing to note is that since an error has occurred, the user would have to intervene in any event, so any attempt at correction is appreciated, even if wrong, as long as the correction does not cause more trouble to correct than the original error. Since DWIM can recognize the difference between trivial corrections, such as misspellings, and serious corrections, such as those involving extensive editing, bad mistakes are usually avoided. When DWIM does make a mistake, the user merely aborts his computation and makes the correction he would have had to make anyway.

\section{Error handling in general}

Certain types of errors that occur in the BBN LISP system cannot be handled by the DWIM program, for example, NON-NUMERIC ARG, an error generated by the arithmetic functions; ARG NOT ARRAY, from the primitive array functions, etc. These are data type errors.* Another class of errors not handled by DWIM are the "panic" errors: BP FULL, a complaint from the compiler meaning it has run out of binary program space; NONXMEM, an attempt to reference nonexistent memory, usually caused by treating an array pointer as a piece of list structure; PDL OVFLW, meaning pushdown list overflow, which usually implies a looping program, etc. Both data type and panic errors are not fixable, but they are helpable.

\footnotetext{
* Sometimes these errors are in fact caused by misspellings, but it is impossible to tell in general.
} 
In our system, whenever an error occurs, it causes a trap to a user modifiable program. (It is through this program that DWIM works.) If DWIM has not been enabled, or if the user aborts an attempted DWIM correction, or if DWIM cannot fix the error that has occurred, the system goes into a "break" and allows the user to interact with the system while maintaining the context of the program in which the error occurred. This allows the user to intervene to try to rectify the problem or to salvage what he can of the computation. While in the break, the system accepts and evaluates inputs from the teletype. Since all of the power of the system is available to him, the user can examine variables, change their values, define and evaluate new functions and even edit functions he is currently in. If another error occurs in a computation requested while in the break, the system goes into a second, lower break, and so on. Thus it is rarely the case that the results of a lengthy computation are lost by the occurrence of an error near its end.

The following example illustrates this process (user input is preceded by ":” or “*”). The user is running a large, compiled system, one of whose subroutines is to perform the alphabetization of a list of names. The first indication of something wrong in the system is the error message ATTEMPT TO CLOBBER NIL, meaning the program is attempting to change the value of NIL (not allowed in our system). The system goes into a break (1), and the user determines where the error occurred by requesting a backtrace (2). He sees that he is inside the function ALPHA and interrogates the value of some of ALPHA's variables (3). He realizes that the problem arose when his alphabetization routine attempted to compare the last element in the list to the one following it, i.e. an end-check problem. Still in 143 the break, he proceeds to call the editor on the function ALPHA (4). DWIM corrects his spelling, and since ALPHA is compiled, the editor retrieves its defining symbolic expression from its property list, typing PROP (5) to call this to the user's attention. Consulting his listing, the user instructs the editor to find the expression beginning with COND that contains RETURN, (6) which he then examines (7). The expression he wants is the one before the COND, so he backs up (8), and makes the appropriate correction (9). He then recompiles ALPHA (10). Notice he is still in the original break.

ATTEMPT TO CLOBBER NIL

TEITELMAN

(RPLACA BROKEN)

WHERE? $\quad 2$

RPLACA

ALPHA

ACCOUNTS2

ACCOUNTS1

ACCOUNTS

:X

(BOBROW OUILLIAN MURPHY BELL NIL) 3

$: Y$

(NIL)

$: Z$

TEITELMAN

EDITF(ALHPA) 4

$=A L P H A$

PROP

EDIT

*(COND CONTAINING RETURN)

PP

(COND

(FLG (GO LP))

$(T(R E T U R N X)))$

${ }^{*} \mathrm{BACK}$ PP

2

(NULL (SETQY (CDR Y)))

*(EMBED SETQ IN CDR) 


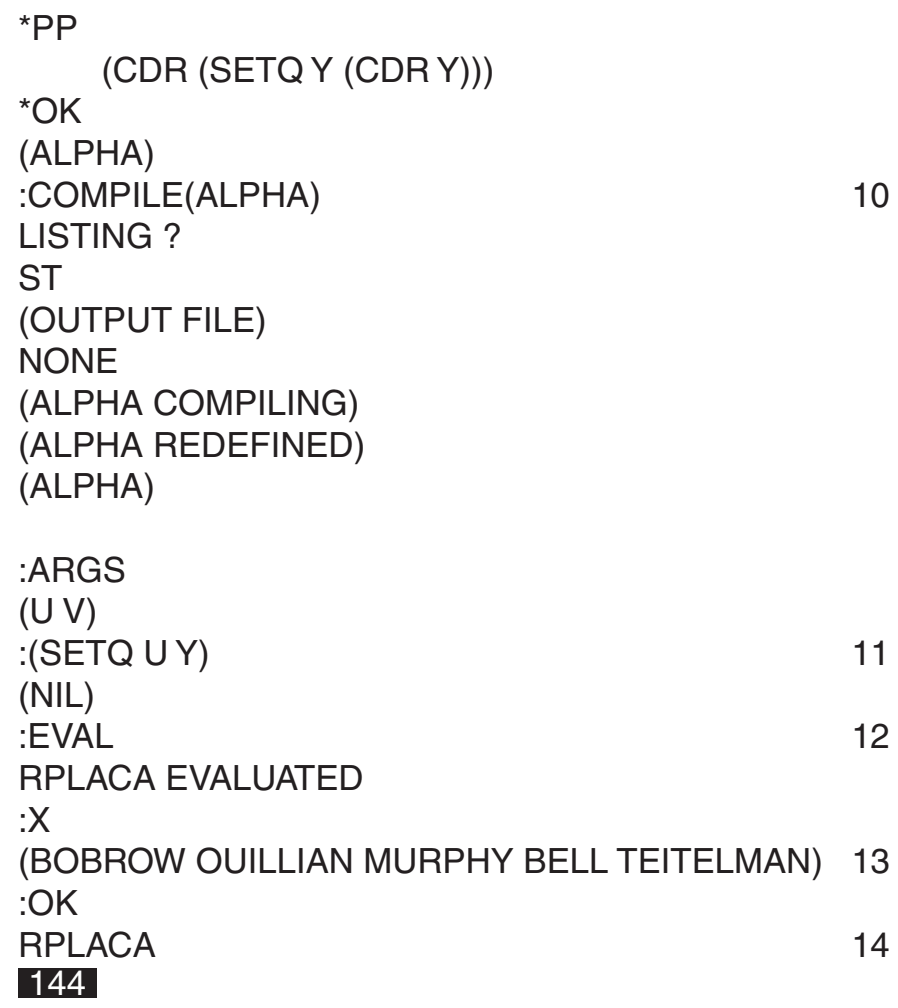

Now the user wishes to continue the computation. He must therefore correct the immediate problem in the function RPLACA i.e. its first argument is NIL. He does this by changing (setting) the first argument, U, to Y (11). He then evaluates RPLACA (12), double checks it by looking at X, and releases the break by typing OK (14).

As illustrated above, when an error occurs a user invariably wants to look back and see what happened earlier in the computation to cause the error situation. In BBN LISP, all information regarding the state of the computation in progress is stored on the push-down list and is explicitly available to the user and to user programs. In fact, without this capability, DWIM could be used only to correct certain trivial errors. We believe that for any type of programming laboratory environment, it is absolutely essential that programs be able to examine the state of the world at any point in the computation. In terms of LISP, this implies being able to examine the sequence of functions that have been called and to look at variable bindings. Since the same variable may be bound a number of times in nested function calls, especially during a recursive computation, the program must be able to specify which binding of a variable it is referencing and be able to change a particular binding if necessary. For example, had $\mathrm{X}$ and $\mathrm{Y}$ been the names of RPLACA's arguments, the user should still be able to interrogate the value of $\mathrm{X}$ and $\mathrm{Y}$ in ALPHA while inside RPLACA. Finally, the user or program must be able to cause the computation to return back to a specified point, i.e. to a particular function on the push-down list, regardless of the number and type of intervening functions that have been called. All of these capabilities are present in our system.

\section{User breaks}

The capability of stopping a computation and maintaining its context while executing teletype inputs is also directly available to the user in a variety of forms as an aid in debugging (see Reference 2). In the simplest case, the user can request that selected functions be modified to cause breaks whenever they are called, or only when a certain condition is satisfied; for example (BREAK ALPHA (GREATERP (LENGTH X) 10)) will cause the alphabetization routine to break whenever it is given a list of length greater than 10. At this point the user can intervene and examine variables, edit functions, etc. exactly as in the case shown above when an error occurs and the system causes a break.

Another way of using the break feature is to specify that a function be "broken" only when it is called from some particular function. For example, the user would be reluctant to break on the function SETQ, since almost every function uses it and the system would forever be going into a break. However, he could (BREAK (SETQ IN ALPHA)), 
which would break only on those calls to SETQ from within ALPHA. In this case, the performance of SETQ is not affected or degraded when called from any other function.

The user can also request that breaks be inserted at specified points inside a function. The editor is then called (in this case the function must be an interpreted one, i.e. have an S-expression definition) to find the appropriate point and insert the break. For example, the user could break in ALPHA (BEFORE(COND CONTAINING RETURN)), which would cause a break just before executing the indicated form.

Finally, the user can request a break at any time during a computation by simply depressing a special key on the teletype. The next time a function is called, usually within a few milliseconds, a break will occur and again the user can intervene and examine the state of the computation, etc. These $\mathbf{1 4 5}$ capabilities are invaluable for localizing problems in complex programs, especially recursive ones, and are powerful tools for finding where to make changes that complement those described below that provide how to make changes.

\section{Advising}

PILOT was originally motivated by the difficulties encountered in using computers for solving very hard problems, particularly those in the area of artificial intelligence (see Reference 9). These problems can be characterized as being extremely difficult to think through in advance, that is, away from the computer. In some cases, the programmer cannot foresee the implications of certain decisions he must make in the design of the program. In others, he can compare several alternatives only by trying them out on the machine. Even after he gets his program debugged, he continues to make alterations to see their effects. Only by experimenting with his working program can he evaluate its performance or hope to extend its generality. Since he cannot accurately predict the effect of changes on the behaviour of the program because of its size and complexity, he must adopt the more pragmatic policy of "let's try it and see what happens." In short, he must be able to treat the computer as his laboratory.

Unfortunately, making changes in programs, especially large and complex programs, is often not a simple matter. Since they may require so much effort, many experimental changes are simply not implemented, with the result that programs soon become "frozen." For this reason, considerable attention and effort in the design and development of PILOT has been devoted to the problem of making changes. One of the results is the concept of advising

The operation of advising consists of modifying the interface between individual functions in a program, as opposed to modifying the functions themselves, which is called editing. The advantage of advising is that it allows the user to treat sections of his own (or some-one else's) program as black boxes, and to make modifications to them without concern for their contents. Since each modification is itself a small program, and modifications can be inserted so as to operate either before or after the original function would be run, advising is a very general and powerful concept.

Advising is carried out in LISP by creating a new function definition in which the original function definition is embedded, and surrounded by the "pieces of advice." This procedure is completely general: the function being advised can be arbitrarily large or small, complex or simple, compiled or interpreted, a system function or one of the user's own.

The individual pieces of advice are each LISP expressions, and so they too are completely general. Thus a piece of advice may simply change the value of some variable or, at the other extreme, request a lengthy computation including perhaps calling the entire advised function recursively. Advice can also be given so as to bypass the entire advised function.

For example, the user could have repaired the problem in ALPHA shown earlier by giving the appropriate advice to RPLACA instead of editing ALPHA. Since RPLACA is called from many functions, the user would probably want to advise RPLACA IN ALPHA:

\section{ADVISE((RPLACA IN ALPHA) (COND ((NULL U) (SETQ U Y))))}

As with the corresponding break, this would modify only the call to RPLACA from within ALPHA.

This operation demonstrates the advantage of advising. It allows the user $\mathbf{1 4 6}$ to make on-line modifications quickly and simply. In addition to using it for correcting bugs, the user can perform modifications for the sake of experimentation, undo the modifications if he wishes, try out other configurations, etc., all without disruption to his high level, 
problem oriented train of thought. Such disruption usually follows when implementing changes requiring a lengthy sequence of operations.

Note that advising complements rather than competes with editing as a way of making changes. In the early stages of debugging, the user is primarily attending to local phenomena in his program and thus may find it natural to make changes by editing. In later stages, he considers his program more in terms of what each piece does, rather than how it does it, and here advising is the tool he wants to use for making changes.

Advising as a tool for modifying the system

Advising not only provides the user with a convenient tool for making changes in his own programs, but also with the means for experimenting with and tailoring the system to his own particular tastes. For example, suppose a user wished to modify PRETTYPRINT to print comments along the right hand side of the page, where a comment was to be indicated as an expression beginning with the atom *. Knowing that SUPERPRINT is the function that "does the work" of prettyprinting, he could

\section{ADVISE(SUPERPRINT (COND ((EQ (CAR E) (QUOTE *)) (RETURN (COMMENT E)))))}

and then define the function COMMENT to do the appropriate formatting.*

Admittedly this particular piece of advising requires the user to have some detailed knowledge of the workings of PRETTYPRINT. However, the important point is that by using ADVISE, changes can be easily effected, even with system functions where changes were not anticipated.

Conversational input

PILOT can be viewed as an interface between the user and his programs. The following somewhat oversimplified diagram illustrates the user-PILOT program configuration:

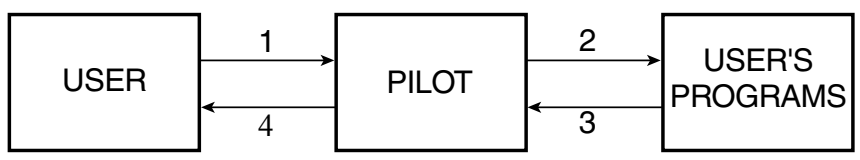

Figure 1

Most of the effort in PILOT is concentrated at interface 2 and 3. However, in order to be really effective, a programming laboratory should not only provide the means whereby changes can be effected immediately, but also in $a$ way that seems natural to the user. Accordingly, we have been experimenting with an English-to-LISP translating program that operates at interface 1, and translates the users requests to PILOT into the appropriate LISP computation. For example, the user could have effected the advice on RPLACA IN ALPHA shown above by:

\section{7}

TELL (RPLACA IN ALPHA): IF ITS FIRST ARGUMENT IS NIL THEN SET IT TO Y.

The following dialogue gives the flavour of user-PILOT interactions obtained with this program. User input is preceded by ">".

PI LOT(T)

PROCEED:

>TELL PROGRESS: IF THE CANNIBALS OUTNUMBER THE MISSIONARIES ON SIDE1,

OR THE CANNIBALS OUTNUMBER THE MISSIONARIES ON SIDE2, THEN RETURN FALSE.

\footnotetext{
*The comment feature is now a part of our system. However, it was initially introduced in precisely this way, in order to evaluate its usefulness. Advising thus provides system designers with a quick means for trying out new features.
} 


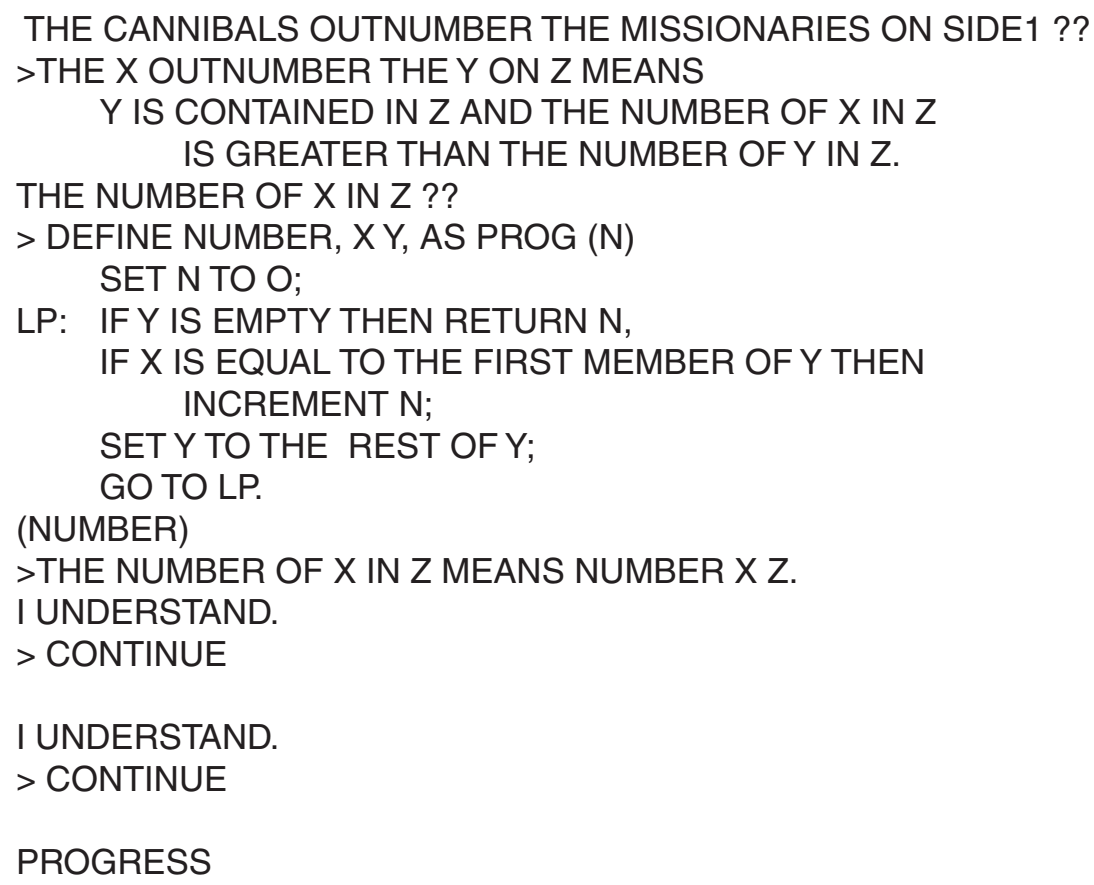

The user instructs PILOT to advise the function PROGRESS with the statement beginning "TELL PROGRESS:". PILOT recognizes this form of request, but does not understand the part about outnumbering. The user then attempts to explain this with the input beginning THE X OUTNUMBER THE Y. This statement will cause an addition to PILOT's already fairly extensive capability for converting English statements to LISP, so that PILOT will be able to understand expressions of this type encountered in the future. However, PILOT cannot interpret the phrase THE NUMBER OF X IN Z in this explanation and so interrogates the user at this lower level. At this point, the user defines a new function NUMBER and then explains the troublesome phrase in terms of this function. PILOT responds that it "understands." The user then instructs PILOT to continue with what it was doing, namely translating the explanation of OUTNUMBER. When this is completed, the user instructs PILOT to continue with the original request, which PILOT now successfully completes.

The current English-to-LISP translator contains a large assortment of useful, if $a d$ hoc, transformational rules written in FLIP (see Reference 10), a string processing language embedded in the BBN LISP system. The set of FLIP rules can be easily expanded or modified. For example, the dialogue shown above resulted in rules for transforming expression of the form THE X OUTNUMBER THE Y ON Z and for THE NUMBER OF X IN Z being added to the translator.

In addition to the FLIP portion of the translating program, there is a post-processor which allows intermingling of LISP expressions with the English, as well as a sort of pidgin-LISP which looks like LISP with the parentheses removed. The translator also contains specialized information for dealing with quantifiers and and/or clauses. For example, the following expressions will be translated correctly into the equivalent LISP forms: NO MEMBER OF X IS ATOMIC AND NOT NULL

\section{8}

THE FIRST ELEMENT OF X IS GREATER THAN THE SECOND AND NOT LESS THAN THE THIRD

THE FIRST ELEMENT OF SOME MEMBER OF X IS A NUMBER THAT IS GREATER THAN THE SECOND ELEMENT

The translator also "remembers" certain contextual information such as what was the last operation requested, what function it referred to, etc. For example: 
FOO

$>$ WHAT IS ITS SECOND ARGUMENT?

Y

We are not asserting that English is a good or even desirable programming language. However, if the user is thinking about his programs in English, then providing him the facility for expressing requests in English will allow him to concentrate more fully on the problem at hand.

\section{Improving PILOT}

"PILOT is the result of an evolutionary process extending over more than two years. However, there is no reason to assume that this process has terminated, nor that PILOT has reached some sort of ultimate state" (see Reference 9). This statement was written in my Ph.D. thesis three years ago and, in the elapsed time, many of the goals established for improvements and additions to PILOT have been realized in our present system. But the statement is still true, and the process still continues.

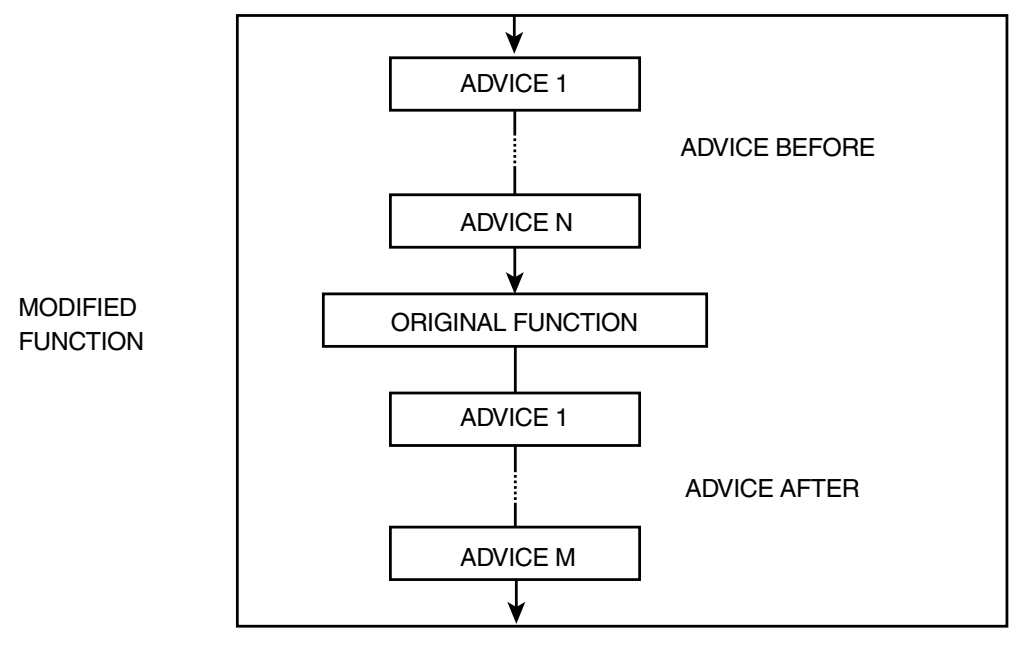

Figure 2

One area of current interest is that of program-writing programs. Programming languages are currently designed to allow the programmer to express the operations he wants the computer to perform in a simple and concise fashion. However, often the programmer may not know precisely what operation he wants the computer to perform, although he may have a clear idea of what he wants the program to accomplish. That is, he may be able to describe its output, or the changes it should make in a data 149 structure. This is not to say that the programmer could not construct the program. However, a system which could accept more goal-oriented descriptions of tasks and produce programs to accomplish them, even if only effective for simple, subroutine-level tasks, would further free its users for high-level operations. Such a system would require a fair degree of problem solving capability and should have a sufficiently rich store of information about programming and programs to enable it to determine similarities in tasks. It should be able to adapt previously written or constructed programs to a new task. In other words, we are trying to construct a system that can handle more of the routine aspects of programming in order to free the human to concern himself more with the creative aspects of the problem.

This is the basic philosophy of the PILOT system: let the computer do it. The significance of PILOT is that it demonstrates the feasibility and desirability of this approach. Even in its current form, PILOT clearly shows that it is possible to get computers to participate in, and co-operate with, research efforts in programming to a much greater extent than is now being done.

\section{References}

1. Bobrow, D. G., Murphy, D. L., and Teitelman, W. The BBN LISP System, April 1969. 
2. Bobrow, D. G. and Teitelman, W. Debugging in an on-line interactive LISP, November 1967. Bolt Beranek and Newman Inc.

3. Engelman, C. "MATHLAB 68” IFIP Congress 68, pp. B91-B95.

4. Johnson, T. E. "Sketchpad III: a computer program for drawing in three dimensions," Proc. SJCC, Spartan Press, Baltimore, Maryland. 1963.

5. Martin, W. A. Symbolic Mathematical Laboratory, Doctoral dissertation, MIT, Cambridge, Massachusetts, January 1967 (also Report TM-36, Project MAC, MIT).

6. Maurer, W. D. "Computer experiments in finite algebra," Comm. ACM, 9, No. 8, August 1966, pp. 589-603.

7. Reintjes, J. F. and Dertouzos, M. L. "Computer-aided design of electronic circuits," presented at WINCON Confer., Los Angeles, California, 2-5 February 1966.

8. Sutherland, I. E. "Sketchpad: a man-machine graphical communication system," Proc. SJCC, Spartan Press, Baltimore, Maryland. 1963.

9. Teitelman, W. PILOT: a step toward man-computer symbiosis, Doctoral dissertation, MIT, Cambridge, Massachusetts, June 1966 (also Report TR-32, Project MAC, MIT).

10. Teitelman, W. Design and implementation of FLIP, a LISP format directed list processor; BBN Report No. 1495, July 1967.

\subsection{Design of high reliability continuous operation systems}

by

W. Ulrich

\section{Scope of problem}

To serve telephone customers in switching systems being installed at a present rate of several hundred thousand lines per year and ultimately at a rate of several million lines per year. The electronic control for these systems must be fully operational in both software and hardware respects to be able to give any kind of service to its customers. Clearly, therefore, there $\mathbf{1 5 0}$ is a need for a very high degree of software as well as hardware dependability.

\section{Characteristics of ESS control program}

Large

The program for the more complex ESS installations is in excess of 220,000 37-bit instructions. Probably half of these instructions are for maintenance while the rest are for telephone operation control. A program of this size was written by a large number of individuals with all the interface problems resulting from such a procedure.

\section{Read only and read/write memories}

The program and certain categories of very rarely changed information are stored on a read only (off-line write) memory. A more conventional read/write memory is used for more frequently changed data and for input/output data.

\section{Common data base}

Many programs access and alter certain types of common data, including common scratch data, common switching network control data, and constants of the office. Moreover, a single short segment of memory is devoted to each call in progress and this segment is also accessed by many different programs.

\section{Pointers}

For reasons of efficiency, a large number of pointers are used. One of the biggest problems comes when a pointer is inadvertently overwritten with completely different data. Such an action permits a rapid propagation of invalid information through the read/write memory of the system and often results in the complete denial of a telephone function. 
Real time problems

The program operates on two levels_ an input/output level, which must keep up with the incoming customer signals, and a base level processing the work that results from these signals. In general, the processing of a call requires a large number of separate and separated program actions which must be separated in real time because further action is required by the customer or by the peripheral system before work can be continued. A call may take 14 seconds of elapsed time but require only about 35 milliseconds of active processing time.

\section{Scheduling algorithm}

Requests for work to be performed are discovered by input/output programs which place these requests in a hopper. Periodically these hoppers are examined and, with the single exception of the service request hopper, all hoppers are emptied completely whenever they are examined. As a means of controlling the load, no more than a fixed number of service requests, i.e. requests for new telephone connections, are handled each time the service request hopper is examined. This is a very convenient way of avoiding excessive overload and responds to such overload in a very good way, i.e. by accepting only a limited amount of new business. Hoppers also provide means for differentiating high priority work, such as effecting the transition from a ringing to a talking connection, from low priority work, such as disconnecting a call.

\section{1}

Need for real time efficiency

The system which was designed using 1960 technology components has a 5.5 microsecond memory cycle time. The requirements of the Bell System are such that it is important that the capacity of the system be at least 60,000 calls per busy hour and preferably considerably more. Therefore, there is a very strong need to keep down the average number of cycles required to process each call and there is a very high payoff for a programming scheme which minimizes this number of cycles.

\section{Electronic memory map control of the switching network}

When new paths are to be selected in the switching network, an image of the network in the read/write memory, containing the busy/idle status of all links, is examined in order to find a new path. Thus, new paths are selected on the basis of a memory image, not directly on the basis of the physical status of the network.

\section{Signal processor}

In larger offices, a separate signal processor controls I/O actions. This processor communicates with the central processor via its own memory which is accessible to the central processor. It generates the same types of hopper entries that are generated by the central processor in the interrupt level activity in smaller offices.

\section{Types of data}

\section{Generic program}

An identical program is placed in all offices of a particular class. (At present there are only five such classes.) The program is arranged so that the variations in features, size, types of inter-office trunk circuits, layout of memory, are handled through a different set of tables of office characteristics. The program itself is identical in its binary form.

\section{Office characteristics}

The tables of office characteristics define the parameters of the particular office and allow the generic program to work properly in the equipment and telephone feature environment of that office. Some of this data is used, for example, to initialize memory and to indicate where various blocks of memory begin and end. Most of this data remains intact until a major addition or major new feature offering (such as a block of memory or a frame of network equipment) is made to the office.

\section{Customer and inter-office data}

This data describes the numerical data of a customer, such as his telephone number and his location on the switching network, and gives details concerning the special services which he has been offered. Inter-office data includes an indication of which group of inter-office trunks is associated with any given office code, the method of signalling over these trunks, the identification of all the members of given trunk groups and the network location of all these trunks.

\section{NATO SOFTWARE ENGINEERING CONFERENCE 1969}


All of this data is normally kept in the read only memory; however, small portions of it may be modified on a day-today basis and are periodically put into read only memory by an off-line process.

\section{Supervisory and per call data}

This data gives the busy/idle status of all customers and trunks and details of all connections that are in the talking state.

\section{2}

\section{Transient data}

This data includes data about calls that have not yet reached the talking state, e.g. calls in the middle of dialing, and usually requires frequent updating.

\section{Signal processor data}

This data, all of which is kept on a read/write store with appropriate back up on the read only store, consists of instructions for the signal processor and data necessary to control I/O actions.

\section{Scratch data}

Scratch data is somewhat different in this environment since it is kept separate from the program and is frequently shared among many different programs.

\section{Techniques for reliability}

On-line checks for data consistency

Relatively little of this is done since such checks require the expenditure of a significant number of extra program cycles and therefore reduce the capacity of the system.

Audits

Most of memory is audited as a matter of routine to check for the consistency of most of the data. In particular, pointers and the memory status of all customer lines and inter-office trunks are audited. Call control segments are audited to make sure that, in case of trouble, the customers controlled by these segments can be released so that they can reoriginate their call. The network map is audited for consistency. Tables of constants, stored for efficiency in read/write store, are checked. Signal processor data and programs are audited.

\section{Emergency audits}

When it is apparent to the system that substantial memory mutilation has taken place, a number of audits are called in and call processing stops until these audits have been completed. These audits attempt wherever possible to retain calls that have already been set up and restore other customers at least to a state from which they may originate new calls and receive terminating calls.

\section{Problems}

\section{Difficulty of discovering overload and mass memory mutilation}

The indication most frequently used that mass memory mutilation has occurred is the failure to complete all required call processing work within a reasonable time. This is also the characteristic of heavy overload. We have found it difficult to distinguish between the two.

\section{Escalation of emergency audits}

When emergency audits are called, they are called in phases such that each audit takes progressively more drastic steps to remove the source of the memory mutilation. We have had problems in which the first phase of the emergency audit should have cleared up the problem but created further symptoms of overload or trouble such that subsequent phases were automatically initiated even though the cause of the memory mutilation had $\mathbf{1 5 3}$ already been cleared up. The final phase, which includes network initialization, is one we particularly try to avoid. 
Recovery from emergency audit

The emergency audit leaves the system in a state resembling that of a crash overload since a number of customers have been disconnected and are trying to re-originate and traffic has been blocked out during the audit. Originations can be admitted slowly but there is more difficulty in limiting the response to seizures from inter-office trunks that have calls destined for this office dialled by customers in another office.

\section{Manual interference}

The single biggest source of problems, as might be expected, has been improper actions by maintenance craftsmen that cause the system to perform emergency audits. Furthermore, it is difficult for the craftsman to get experience so that he can make a good judgment of whether or not to advance to a particular phase and how long he should wait for the system to try to straighten out the problem automatically.

\section{Overloaded offices}

There has been a strong temptation on the part of many telephone operating companies to apply more load to the office than it is capable of handling. Under these circumstances, because of the sensitivity of the system to overload, emergency audits are performed more frequently and recovery following an emergency audit takes longer.

\section{Memory protection}

No memory protection exists in the system and therefore the propagation of poor data arising from incorrect pointers cannot be readily controlled except where out-of-range addresses are generated.

\section{Results}

\section{Unsatisfactory offices}

A small number (in the order of 20 per cent) of the offices have had a significant number of emergency audits for some weeks after they were cut over to service. In some cases, as many as a dozen separate occasions occurred during the first month of service; however, all of these offices, including those in which too much load was applied, have performed well after an initial bad period of as much as one week and a subsequent period of one or two months that was less than satisfactory. In most cases the unsatisfactory offices presented some new characteristics of load that could not be adequately simulated in the laboratory. The unsatisfactory offices are primarily offices with signal processors that have a larger load and more complex interfaces than the offices with no signal processor and whose programs are newer and therefore less thoroughly shaken down.

\section{Satisfactory offices}

The large bulk of the offices installed have an emergency audit rate of about one or two per year after the first three or four months. It is hoped that this number will decrease with time but we must recognize that new features are constantly being added to the program so that the program is not necessarily monotonically approaching the perfectly debugged stage. 


\section{APPENDIX 1 \\ Participants in NATO Conference on Techniques in Software Engineering, Rome, 27th to 31st October 1969}

Chairman: Professor P. ERCOLI,

Facolta di ingegneria,

Via Eudossiana 18,

I-00184 Rome, Italy.

Co-Chairman: Prof. Dr. F. L. BAUER,

Mathematisches Institut der Technischen Hochschule,

D-8 München 2 ,

Arcisstraße 21, Germany.

Mr. J. D. ARON,

IBM Corporation,

18100 Frederick Pike,

Gaithersburg, Maryland 20760, USA.

Prof. R. S. BARTON,

Computer Science

Merrill Engineering Building,

University of Utah,

Salt Lake City, Utah, USA.

Dr. R. BAYER,

Boeing Scientific Research Laboratories,

P.O. Box 3981,

Seattle, Washington 98124, USA.

Mr. R. W. BEMER,

General Electric Company,

Information Systems Group,

13430 Black Canyon Highway, M-2,

Phoenix, Arizona 85029, USA.

Prof. L. BOLLIET,

IMAG,

CEDEX 53,

F-38 Grenoble, Gare, France.

Mr. P. BRINCH HANSEN,

A/S Regnecentralen,

Falkoneralle 1,

DK-2000 Copenhagen F.

Denmark.

Mr. H. M. BROWN,

IBM UK Laboratories,

Hursley, Winchester, England.

Dr. W. S. BROWN,

Bell Telephone Laboratories, Inc.

Murray Hill, New Jersey 07974, USA.

Mr. P. BURKINSHAW,

ICL,

Brandon House,

Broadway,

Bracknel1, Berks., England.
Prof. J. N. BUXTON,

School of Computer Science,

University of Warwick,

Coventry, CV4 7AL, England.

Mr. A. CARACCIOLO DI FORINO,

IEI-CNR,

Via S. Maria 46,

I-56100 Pisa, Italy.

Mr. C. T. CLINGEN,

General Electric Company,

Cambridge Information Systems Laboratory, 575 Technology Square,

Cambridge, Massachusetts 02139, USA.

Dr. E. E. DAVID, Jr.,

Bell Telephone Laboratories, Inc.,

Murray Hill, New Jersey 07974, USA.

Prof. Dr. E. W. DIJKSTRA,

Department of Mathematics,

Technological University,

Postbox 513,

Eindhoven, The Netherlands.

Dr. H. DONNER,

Siemens AG, Dv T SIP

D-8000 München 25,

Hofmannstraße 51, Germany.

Dr. K. EBBINGHAUS,

IBM Laboratories,

Dept. 767,

D-703 Böblingen,

Schönaicher First 10, Germany.

Dr. M. ENGELI,

FIDES Trenhand-Vereinigung,

Bleicherweg 33,

CH-8002 Zurich, Switzerland.

Mr. A. D. FALKOFF,

IBM Scientific Center,

T. J. Watson Research Center,

P.O. Box 218

Yorktown Heights, New York 10598, USA.

Prof. J. A. FELDMAN,

Computer Science Department, Stanford University,

Stanford, California 94305, USA.

Prof. R. W. FLOYD,

Computer Science Department, 
Stanford University,

Stanford, California 94305, USA.

Prof. B. A. GALLER,

Computing Center,

1000 North University Building,

University of Michigan, Ann Arbor, Michigan 48104, USA

Dr. G. GOOS,

Rechenzentrum,

Technische Hochschule,

D-8 München 2,

Arcisstraße 21, Germany.

Prof. C. C. GOTLIEB,

Institute of Computer Science,

Sandford Fleming Building,

University of Toronto,

Toronto, Ontario, Canada.

Prof. J. W. GRAHAM,

University of Waterloo,

Department of Computer Science,

Waterloo, Ontario, Canada.

Dr. H. J. HELMS,

Northern Europe University Computing Center,

Technical University of Denmark,

DK-2800 Lyngby, Denmark.

Prof. C. A. R. HOARE,

Department of Computer Science,

Queens University,

Belfast, BT7 INN, Northern Ireland.

Dr. F. HOFMANN,

Siemens AG, E54,

D-852 Erlangen,

Günther-Scharowsky-Straße, Germany.

Mr. M. E. HOPKINS,

IBM Corporation,

T. J. Watson Research Center,

P.O. Box 218,

Yorktown Heights, New York 10598, USA.

Mr. M. ITALIANI,

Syntax,

Via Camperio 3,

Milan, Italy.

Dr. H. KANNER,

Technology Development Department,

Control Data Corporation,

3145 Porter Drive,

Palo Alto, California 94304, USA.

Mr. P. M. KJELDAAS,

Kjeller Computer Installation,

P.O. Box 70, N-2007 Kjeller, Norway.

Dr. K. LAGALLY,

Rechenzentrum,

Technische Hochschule,

D-8 München 2,

Arcisstraße 21, Germany.
Dr. B. W. LAMPSON,

Department of Computer Science,

University of California,

Berkeley, California 94720, USA.

Mr. C. A. LANG,

University Mathematical Laboratory,

Corn Exchange Street,

Cambridge, England.

Dr. A. LEMMA,

Selenia s.p.a.,

Via Tiburtina Km. 12,400,

Rome, Italy.

Mr. E. S. LOWRY,

IBM Corporation,

Systems Development Division,

Building 706, Department D76,

Poughkeepsie, New York 12602, USA.

Dipl. ing. P. LUCAS,

IBM Laboratory,

Parkring 10,

Vienna 4, Austria.

Dr. R. M. McCLURE,

3741 Alta Vista Lane,

Dallas, Texas 75229, USA.

Mr. J. M. MacGOWAN, Jr.,

UNIVAC Data Processing Division,

2276 Highcrest Drive,

Roseville, Minnesota 55113, USA.

Dr. R. M. NEEDHAM,

University Mathematical Laboratory,

Corn Exchange Street,

Cambridge, CB3 2SG, England.

Mr. M. D. OESTREICHER,

Computer Analysts and Programmers Ltd., CAP House,

14/15 Great James Street,

London, W.C.1, England.

Prof. A. J. PERLIS,

Department of Computer Science,

Carnegie Mellon University,

Pittsburgh, Pennsylvania 15217, USA.

Prof. B. RANDELL,

The Computing Laboratory,

University of Newcastle upon Tyne,

Claremont Tower,

Claremont Road,

Newcastle upon Tyne, NE1 7RU, England.

Dr. J. C. REYNOLDS,

Applied Mathematics Division,

Argonne National Laboratory,

Argonne, Illinois 60439, USA.

Mr. D. T. ROSS,

SofTech, Inc.,

391 Totten Pond Road,

Waltham, Massachusetts 02154, USA. 
Mr. J. P. ROSSIENSKY,

Compagnie Internationale pour l'Informatique, Les Clayes/Bois 78, France.

Mr. C. A. SCALZI,

IBM Corporation,

Building 705,

Poughkeepsie, New York, 12602, USA.

Dr. H. SCHORR,

IBM Corporation,

T. J. Watson Research Center,

P.O. Box 218,

Yorktown Heights, New York 10598, USA.

Mr. J. 1. SCHWARTZ,

c/o King Resources,

12011 San Vicente Blvd.,

Los Angeles, California 90049, USA.

Dr. G. SEEGMÜLLER,

IBM Laboratories,

Dept. 770 ,

D-703 Böblingen,

Schönaicher First 10, Germany.

Mr. I. P. SHARP,

P.O. Box 71 ,

Toronto-Dominion Centre,

Toronto 111, Canada.

Mr. T. H. SIMPSON,

IBM Corporation,

11141 Georgia Avenue,

Wheaton, Maryland 20902, USA.

Mr. C. STRACHEY,

Oxford University Computing Laboratory,

45 Banbury Road,

Oxford, England.

Dr. W. TEITELMAN,

Bolt, Beranek and Newman, Inc.,

50 Moulton Street,

Cambridge, Massachusetts, USA.

Dr. W. ULRICH,

Bell Telephone Laboratories Inc.,

Room 2C-235,

Naperville, Illinois 60540, USA.

Prof. dr. ir. W. L. VAN DER POEL,

Delft University of Technology,

Department of Mathematics,

132 Julianalaan,

Delft, The Netherlands.

Mr. S. WARSHALL,

Applied Data Research,

450 Seventh Avenue,

New York, New York 10001, USA

Dr. H. R. WIEHLE,

c/o AEG/Telefunken,

D-775 Konstanz, Postfach 154, Germany.
Dr. HANS-W. WIPPERMANN,

D-7500 Karlsruhe,

Rechenzentrum der Universität,

Englerstr. 2, Germany.

Prof. N. WIRTH,

Eidg. Technische Hochschule,

Fachgruppe Computer-Wissenschaften,

Leonhardstr. 33,

CH-8006 Zurich, Switzerland.

Mr. M. WOODGER,

Ministry of Technology,

National Physical Laboratory,

Division of Computer Science,

Teddington, Middlesex, England.

Scientific Secretaries:

Mr. R. ELLIS,

ICL,

30-31 Friar Street,

Reading, RG1 1DX, England.

Mr. I. HUGO,

Infotech Ltd.,

Nicholson House,

High Street,

Maidenhead, Berkshire, England.

Observers:

Mr. P. H. KENNEY,

DACOS,

ADP Division,

SHAPE,

B-7010, Belgium.

Mr. L. CARIOU,

Chef du Service Etudes Informatiques,

Compagnie d'Etudes et de Realisations de Cybernetique Industrielle,

22 rue de Charonne,

Paris 11e., France.

Dr. H. HAILER,

Deutsche Forschungsgemeinschaft,

D-53 Bonn-Bad Godesberg,

Kennedy-Allee 40, Germany.

Dr. H. VON ISSENDORFF,

Forschungsinst. f. Funk u. Mathematik,

Werthoven,

Königstraße 2, Germany.

Dr. J. KONTOS,

Electronic Computers Division,

Greek Atomic Energy Commission,

NRC "Democritus",

Athens, Greece.

Col. A. MPATIS,

Operational Research Division,

Hellenic Armed Forces Command,

Athens, Greece.

Ten.Col. M. CARLA,

Istituto Geografico Militar Italiano, 
Via Cesare Battisti 8,

Firenze, Italy.

Lt.Cdr. G. FALCIAI,

Maricensadoc Maristat,

Rome, Italy.

Dr. M. M. PACELLI,

Italsiel,

Via Abruzzi 3,

Rome, Italy.

Mr. G. M. PALERMO,

Italsiel,

Via Abruzzi 3,

Rome, Italy.

Dr. E. ROSSI,

Selenia,

Via Tiburtina Km. 12,4,

Rome, Italy.

Mr. W. LIMBURG,

Laboratory for Electronic Development of the Armed Forces,

Haarlemmerstraatweg 7,

Oegstgeest,

The Netherlands.

Dr. P. C. POOLE,

UKAEA Establishment,

Culham Laboratory,

Abingdon, Berks., England.

Secretaries:

Miss E. I. AUSTIN,

Scientific Affairs Division

NATO,

Brussels 39, Belgium.

Miss M. CHAMBERLIN,

Room S 106,

St. Clements Building,

London School of Economics,

Houghton Street,

Aldwych,

London, W.C.2, England.

Miss A. LAYBOURN,

The Computing Laboratory,

University of Newcastle upon Tyne,

Claremont Tower,

Claremont Road,

Newcastle upon Tyne, NE1 7RU, England.

Scientific Affairs Division:

Prof. G. RANDERS,

Assistant Secretary General for Scientific Affairs.

Dr. H. ARNTH-JENSEN,

Head of Operations.

Mr. F. M. BLAKE,

Consultant on Computer Science. 


\section{APPENDIX 2}

\section{Addresses of Welcome}

Translation of the telegram from Senator G. Bo, Italian Minister for Coordination of Scientific and Technological Research.

To Prof. P. Ercoli, Chairman of NATO Conference on Techniques in Software Engineering.

Thank you for your kind invitation to the Conference on Techniques on Software Engineering to be held in Rome from 27 to 31 October. Please convey to the participants my most cordial welcome and good wishes. I am sure that the work of such outstanding personalities of the scientific world gathered together in Rome will lead to valuable results in this field which is of the greatest importance for scientific research and technological progress.

Bo, Minister of Scientific Research.

Translation of the telegram from Prof. L Dadda, President of the Italian Computer Society (AICA).

To Prof. Ercoli, Chairman of NATO Conference on Techniques in Software Engineering.

On the occasion of the opening of the Conference on Techniques in Software Engineering may I ask you to extend a hearty welcome to all participants and wish them a pleasant stay and a stimulating exchange of ideas in this most important field of engineering.

Dadda, President of the Italian Computer Society. 


\section{CONTRIBUTOR INDEX}

The index gives the page number of the named quotations, and in bold face, of papers reproduced in full in section 7 . The page number within square brackets indicates where the full name and address of the contributor is given.

Aron $11,23,43,46,47,50,52,53,59,63,68,113,[154]$

Bauer 22, 62, [154]

Bayer 26, 36, 38, [154]

Bemer 22, 30, [154]

Brown, H. M. 53, 58, [154]

Brown, W. S. 23, 29, 31, 33, 46, 80, [154]

Burkinshaw 24, [154]

Buxton 19, 33, 52, 53, [154]

David 12, [154]

Dijkstra 13, 18, 21, 22, 23, 28, 30, 64, 84, [154]

Engeli 15, [155]

Ercoli 27, 64, [154]

Falkoff 17, 18, 22, 47, 53, 63, 66, 88, [155]

Feldman 12, 16, 18, 21, 29, 33, 62, [155]

Galler 12, 22, 24, 26, 62, [155]

Gotlieb 26, 66, 93, [155]

Haller 47, [157]

Hoare 21, 49, 53, [155]

Hopkins 20, 23, 39, 99, [155]

Kanner 60, [155]

Lampson 18, 23, 31, 38, 66, [155]

Lang 18, 23, 46, 101, [155]

Llewellyn 22, [156]

Lowry 16, 25, [156]
Lucas 16, 21, 22, [156]

McClure 63, [156]

MacGowan 52,106, [156]

Needham 11, 24, 63, 111, 113, [156]

Oestreicher 15, 23, 58, 64, [156]

Perlis 17, 21, 22, 24, 25, 28-30, 32, 33, 52, 61, 62, 65, [156]

Poole 33, [158]

Randell 13, 17, 22, 27, 48, [156]

Reynolds 22, 29, [156]

Ross 11, 24, 26, 29, 31, 33, 46, 62, [156]

Scalzi 15, 52, 58, [156]

Schorr 20, 26, 27, 37, 46, 47, 50, 66, 114, [156]

Schwartz 17, 41,122, [156]

Seegmüller 15,16, [156]

Sharp 12, 27, [156]

Strachey 9, 29, 32, 65, [156]

Teitelman 24, 25, 26, 64, 137, [156]

Thompson 46, [157]

Ulrich 12, 48, 49, 53,149, [157]

Warshall 34, 36, 37, [157]

Wirth 17, 18, 20, 24, [157]

Woodger 65, 66, [157] 


\section{SUBJECT INDEX}

This subject index is known to be deficient in many respects, and it is meant merely as a supplement to the detailed list of contents on pages 3 and 4 .

\begin{tabular}{|c|c|}
\hline Abstract data structures 86 & COMMON 31 \\
\hline Abstract machine 15 & Communication Pool 135 \\
\hline Acceptance 47 & Communist 17 \\
\hline ACM SIGOPS 26 & Compiler 81, 99, 114, 122 \\
\hline Activity networks 78 & Compiler-compiler 101 \\
\hline Ad hoc techniques 115 & Complexity 100 \\
\hline AED-O 29, 33, 103 & Computation time clock 26 \\
\hline Aesthetic criteria 90 & Computer Aided Design Project 29 \\
\hline Aircraft surveillance 41 & Computer Learning and Systems Corporation 94 \\
\hline Airline reservations systems 122 & Computer program acquisition 129 \\
\hline ALGOL $6016,24,29,80,86,101,103,134$ & Computer Program Contract End Item 129 \\
\hline ALGOL 68 22, 80 & Computing Reviews 61 \\
\hline Algorithmic approach 132 & Computing Science 9, 65, 113 \\
\hline ALTRAN-F 80 & Confidence level 84 \\
\hline Analytical model 94 & Context free syntax 90 \\
\hline APL 16, 18, 25, 66, 88 & Continuous operation system 149 \\
\hline Apollo 43 & Conversational technique 24 \\
\hline Applied computer science 62 & Core dump 23 \\
\hline Archaeological record 100 & Coupling 30 \\
\hline Army of ants 50 & Creativity 130 \\
\hline Artificial Intelligence 16, 61 & Critical module 29 \\
\hline Assembly language 101, 102 & CTSS 42 \\
\hline Asynchronous process 36 & Curtain cleaner 41 \\
\hline Audit 152 & Customer relations 51 \\
\hline \multicolumn{2}{|l|}{ Automata theory 61} \\
\hline \multirow[t]{2}{*}{ Automated analysis 25} & Data approach 134 \\
\hline & Data collection software 107 \\
\hline Bandwagon 60 & Data consistency 152 \\
\hline Bar chart 78 & Data definition 135 \\
\hline BASIC 62 & Data Flow Specification 17 \\
\hline BBN LISP 137 & Data-independent programming language 42 \\
\hline BCPL 29, 103 & Data representation 29 \\
\hline Binary patch 112 & Debugging 23, 117 \\
\hline Binding 100 & Debugging time 46 \\
\hline Bolt, Beranek and Newman Inc. 139 & Debugging tool 110 \\
\hline Bootstrapping 29, 31 & Decision postponement 37 \\
\hline Break 142 & Decompilation 25, 36 \\
\hline Bug rate 20 & Decouple 25 \\
\hline Burroughs 10, 24 & Deformable 36 \\
\hline \multirow[t]{2}{*}{ Business management system 123} & Demonstration Project 10 \\
\hline & Disease structure 64 \\
\hline Cape Kennedy 43 & Doctors of engineering 64 \\
\hline CASE 94 & Documentation 21, 42, 80, 88, 118, 130 \\
\hline CASTER 54 & Downtime objective 48 \\
\hline \multicolumn{2}{|l|}{ Catholic 17} \\
\hline CDC 6400/6600 82 & Electric toothbrush 24 \\
\hline Centralized data maintenance 42 & Encyclopaedia 66 \\
\hline Chairman of the Board 49 & Envelope 38 \\
\hline Channel utilization 107 & Environmental description 25 \\
\hline Chinese Army approach 88 & Environmental specif ication 29 \\
\hline CLEAR 53, 54, 59, 60 & EPL 103 \\
\hline CLEAR-CASTER 54, 58, 59 & Error diagnostic 117 \\
\hline Closure 30 & ESS 150 \\
\hline COBOL $11,17,30,77,80,101,116,122,134$ & EULER 18 \\
\hline COBOL F compiler 116 & Exec 852 \\
\hline CODASYL 30 & Exhaustive testing 21 \\
\hline \multicolumn{2}{|l|}{ Code economization 18} \\
\hline Code generation 115 & Fallback 112 \\
\hline Command and Control 134 & Fence building 100 \\
\hline
\end{tabular}


File maintenance 111

FLIP 147

Formal design language 88

FORTRAN 10, 22, 25, 29, 30, 31, 36, 44, 52, 77, 80, $101,116,134$

FORTRAN D compiler 117

FORTRAN E compiler 116

FORTRAN G compiler 116

FORTRAN H compiler 116

FORTRAN laundry 37

Functional specification 68

Fundamental concept 65

Gathering 31

GE 625/635 82

Gemini 44

Generalized Programming 31

Global optimization 40

Gödel numbering system 35

GPSS 94

Half-bootstrapping 29

Hardware dependability 150

Hardware design 65

Hardware monitor 26, 94, 107

Hierarchical structure 11

High-level language 74, 102

Holt, A. W. 31

Houston 43

IBM 20, 21, 27, 45, 53, 80, 116

IBM Federal Systems Division 69

IBM S/360 12, 32, 39, 44, 82, 90

IBM 70444

IBM 709039

IBM 709445

ILLIAC IV 27, 32

lliffe, J. K. 24

Inductive proof 21

Informatik 62

Intelligent ignoramus 24

Interface 118

Ivory towers 33

JCL 51, 52, 54

Jet Propulsion Laboratories 43

Job mix 110

Job scheduler 99

Job shop scheduling 94

JOVIAL 17, 134

KDF9 29

Knuth, D. E. 66

Language design 19

Library control system 59

Linkage editor 51

Linking loader 30

LISP 80,101.137

Logistics 99

Loop flattening 37

Loop summarization 109

M6 82

Macbeth 23

Macro 29, 82

Maintenance 118
Malfunction 11

Management science 62

Manned Space Flight Centre 43

Mathematical analysis 47

Mathematical engineer 64

McG 101

Medical School 63

Memory mutilation 48

Mercury 44

Methodology 123

Milestone schedule 131

Mission simulation 45

MIT 28, 29, 42, 128

Mixed language system 105

Modelling 93

Modelling technique 45

Module 23, 24, 38, 45, 53, 87

MOL/940 18,103

Multi-access system 101

MULTICS 12, 42

Multiprocessor interlocking 52

Multiprocessor system 108

NASA 43

NATO Summer School 49

Necklace 87

NELIAC 117

New York City 51

Non-programmer 124

Non-standard interface 39

Numerical analysis 61

Octal dump 112

Off-line debugging 24

Onion shaped 28

On-line debugging 24, 102

Operating system 81, 99, 101, 107, 111

Operations research 62

Optimization 115

Optimization technique 16

Optimizing compiler 100

Orbit determination system 50

OS/360 12, 15, 20, 39, 44, 51, 52, 58, 64, 99

Ossification 61

Oxford 29

Panmunjon 14

Parkinsonian slope 53

PASCAL 20

Pathology 64

PDP10 32

Pearl 87

Performance monitoring 93

PERT 45, 78, 131

Ph.D. programs 61

Pidgin English 50

PILOT 137

Pilot Plant 10

Pipelining 37

PL/1 16, 21, 25, 50, 77, 80, 101, 117, 134

PL/360 18.103

Pointer 49

Portability 28

Portable software 31

Poughkeepsie 59

Predicate 21

Primitive 29, 82, 117 
Problem oriented language 80

Process description 16

Product test 21

Professional standards 32

Program complexity 21

Program correctness 84

Program Logic Manual 99

Program module 15

Program reliability 47

Program testing 85

Program verification 21

Programmer productivity 72

Programming laboratory 137

Programming Project Management Course 69

Programming systems management 52

Proofs of equivalence 89

Protection ring 28

Pun 32

Puritans 49

Real time clock 26

Real time simulation 47

Real time system 44

Recursion 9

Register allocation 116

Release 20

Representational commitment 34

Reprogramming 42

Resource estimating 71

RTOS 44

Run-time system 102

S3 94

SABRE 39

SABRE PL/ 139

SAGE 41,122

SAL 103

Scaffolding 116

Schedule 124

Schedule control procedure 45

Scheduling 116

School teacher 41

Second Conference on Applications of Simulation 94

Semantics 89

Shakespeare 23

Sheriff 40

Sift 37

Side effect 23

SIMSCRIPT 94

SIMULA 17

Simulation 106

Snapshot 23, 25

SNOBOL 80

Software architecture 12

Software dependability 150

Software engineer 111

Software engineering 61,114

Software engineering tool 60

Software instrumentation 107

Software monitor 94

Software performance 109

Software specification language 15

Source text 111

Specification 44, 123

State description 16

Statistics gathering system 45
Street-car conductor 41

Super programmer 50

Supervisory personnel 46

Support 30

Support software 33, 111

Support system 15, 42, 51

Support tool 131

Switching system 149

System architect 70

System checkout tool 135

System crash 113

System generation routine 52

System overhead 107

System performance 106

System programming tool 60

System test 77

System testing 46, 123

System test tool 134

Technical writer 56

Technology transfer 13

Telephone central office 49

Test case 21

Testing 23

Testing by sampling 21

Test specification 42, 132

TEXT/360 58

Text book 65

Time sharing system 42, 94, 124

Top-down approach 130

Transferability 117

Translator writing system 114

Tranportability of data 29

Turanski. W. J. 31

Undertaker 41

Units of work 73

UNIVAC 1108 82, 106

U.S. Air Force 41, 129

U.S. Air Force 375 Management concepts 129

U.S. Department of Defense 129

Vanguard 43

Vienna definition method 15, 21

Warm start mechanism 113

T. J. Watson Research Center 101

Worst Case approach 22 